\title{
Ground States in the Spin Boson Model
}

\author{
David Hasler and Ira Herbst
}

\begin{abstract}
We prove that the Hamiltonian of the model describing a spin which is linearly coupled to a field of relativistic and massless bosons, also known as the spin-boson model, admits a ground state for small values of the coupling constant $\lambda$. We show that the ground-state energy is an analytic function of $\lambda$ and that the corresponding ground state can also be chosen to be an analytic function of $\lambda$. No infrared regularization is imposed. Our proof is based on a modified version of the BFS operator theoretic renormalization analysis. Moreover, using a positivity argument we prove that the ground state of the spin-boson model is unique. We show that the expansion coefficients of the ground state and the ground-state energy can be calculated using regular analytic perturbation theory.
\end{abstract}

\section{Introduction}

The spin boson model describes a quantum mechanical two-level system which is linearly coupled to the quantized field of bosons. We assume that the quantized field is a relativistic field of massless bosons, and we do not impose any infrared regularization. In that case the spin-boson model can be used as a simplified caricature describing an atom coupled to the quantized electromagnetic field. The two-level system is a coarse approximation of the energy levels of the atom. This model has been extensively investigated, see for example $[12,17,23,24]$ and references therein.

Our first result states that for all values of the coupling constant a possible ground state of the spin boson model must be unique. This result is shown using a positivity argument with respect to a suitable choice of measure space.

Our second result is that the spin boson model admits a ground state for small values of the coupling constant. Quantum mechanical systems which are coupled to a relativistic field of massless bosons typically do not admit ground states unless cancellations of infrared divergences occur. The reason the spin boson model admits a ground state originates from the fact that the coupling 
matrix has no diagonal entries, see (2.3). In non-relativistic quantum electrodynamics (qed) the gauge symmetry seems to be responsible for the existence of ground states of molecules [6,10].

Our third and main result is that a suitable choice of the ground state as well as its energy are analytic functions of the coupling constant. In nonrelativistic qed, expansions in the coupling constant of the ground state and its energy have recently attracted attention. In $[3,4]$ it was proven that there exists an asymptotic expansion involving coefficients which depend on the coupling parameter and may contain logarithmic expressions. Other expansion algorithms were employed, for example in $[7,8,15]$ and it was shown that logarithmic terms can occur in non-relativistic qed. On the other hand, it was shown that an atom in the dipole approximation of qed (which effectively leads to an infrared regularization) has a ground state and ground-state energy which are analytic functions of the coupling constant [14]. We hope that our analyticity result concerning the spin boson model will help to shed light on the nature of infrared divergences occurring in such expansions.

Once the analyticity of the ground state and its energy have been shown, it is natural to ask whether the coefficients of their power series expansions can be obtained from regular analytic perturbation theory. We prove that this is indeed the case and illustrate how the ground state and its energy can be calculated using Rayleigh-Schrödinger perturbation theory. To this end we artificially introduce an infrared cutoff in the Hamiltonian and show that the ground state and the ground-state energy are continuous functions of that cutoff. Validity of Rayleigh-Schrödinger perturbation theory will then follow from the uniqueness property of the ground state. In view of the explicit form of the Rayleigh-Schrödinger coefficients it is rather surprising that these coefficients are infrared finite. The coefficients are given as a sum of terms. While infrared divergent terms occur our analyticity result implies that the sum of these terms must be finite in the limit when the infrared cutoff is removed.

Under the additional infrared regularization assumption $\omega^{-3 / 2} f \in \mathfrak{h}$ (see Sect. 2 for notation) the existence of a ground state is well known. With suitable infrared assumptions the existence of a ground state can be established using methods from statistical mechanics [24,25], analytic methods [1,6, 12], or operator theoretic renormalization [5,2]. However, in the infrared critical case as considered here the mere existence of a ground state is subtle. Indeed, in related models with a similar infrared behaviour there may not exist a ground state, see for example $[11,16,19]$. An interesting related question in the infrared critical case is whether the expectation of the number operator in the ground state is finite or not.

Let us now address the proof of the main results. The ground-state energy is embedded in the continuous spectrum, see Proposition 3.2. In such a situation regular perturbation theory is typically not applicable and other methods have to be employed. To prove the existence result as well as the analyticity result for the spin-boson model we use a variant of the operator theoretic renormalization analysis as introduced in [5] and further developed in [2]. The analysis as outlined in these papers is not directly applicable to problems 
which are infrared critical. To be able to apply a renormalization procedure, we first perform two initial so-called Feshbach transformations. This converts the spectral problem of the original Hamiltonian into a problem involving sums of normal ordered operators containing only an even number of creation and annihilation operators. We then must prove that on the space of such operators the renormalization procedure converges. To show this in a proper way we have to provide a detailed exposition of the operator theoretic renormalization transformation.

In [14] the analyticity of the ground state as well as the ground-state energy of an atom in the dipole approximation of non-relativistic qed was proven. We want to point out that also in [14] operator theoretic renormalization was used in the proof, with a somewhat different representation of the spectral parameter. Whereas the problem considered in [14] was infrared regular, the problem considered in this paper is not subject to an infrared regularization. Moreover, in [14] the proof made use of the fact that renormalization preserves analyticity in the space of operators while in this paper we use that renormalization preserves analyticity in the space of integral kernels. In the next section we introduce the model and state the main results, which will then be proven in later sections.

\section{Model and Statement of Results}

For a Hilbert space $\mathfrak{h}$ we introduce the bosonic Fock space

$$
\mathcal{F}(\mathfrak{h}):=\bigoplus_{n=0}^{\infty} S_{n}\left(\mathfrak{h}^{\otimes n}\right),
$$

where $S_{n}$ denotes the orthogonal projection onto the subspace of totally symmetric tensors in $\mathfrak{h}^{\otimes n}$, and $S_{0}\left(\mathfrak{h}^{\otimes 0}\right):=\mathbb{C}$. We introduce the vacuum vector $\Omega:=(1,0,0, \ldots) \in \mathcal{F}(\mathfrak{h})$. Henceforth, we fix $\mathfrak{h}$ to be $L^{2}\left(\mathbb{R}^{3}\right)$ and set $\mathcal{F}:=\mathcal{F}(\mathfrak{h})$. We shall identify vectors $\psi \in \mathcal{F}$ with sequences $\left(\psi_{n}\right)_{n=0}^{\infty}$ of $n$-particle wave functions, $\psi_{n}\left(k_{1}, \ldots, k_{n}\right)$, which are totally symmetric in their $n$ arguments, and $\psi_{0} \in \mathbb{C}$. The scalar product of two vectors $\psi$ and $\phi$ is inherited from $\mathfrak{h}$ and is given by

$$
\langle\psi, \phi\rangle=\sum_{n=0}^{\infty} \int \overline{\psi_{n}\left(k_{1}, \ldots, k_{n}\right)} \phi_{n}\left(k_{1}, \ldots, k_{n}\right) \mathrm{d}^{3} k_{1} \ldots \mathrm{d}^{3} k_{n} .
$$

For $g \in \mathfrak{h}$ one associates a creation operator defined as follows. For $\eta \in$ $S_{n}\left(\mathfrak{h}^{\otimes n}\right), a^{*}(g) \eta$ is given by

$$
a^{*}(g) \eta=\sqrt{n+1} S_{n+1}(g \otimes \eta) .
$$

This defines a closable linear operator whose closure is also denoted by $a^{*}(g)$. The annihilation operator $a(g)$ is defined to be the adjoint of $a^{*}(g)$. Formally, we write

$$
a(g)=\int \overline{g(k)} a(k) \mathrm{d}^{3} k, \quad a^{*}(g)=\int g(k) a^{*}(k) \mathrm{d}^{3} k,
$$


where $a(k)$ and $a^{*}(k)$ are operator-valued distributions. They satisfy the so-called canonical commutation relations

$$
\left[a(k), a^{*}\left(k^{\prime}\right)\right]=\delta\left(k-k^{\prime}\right), \quad\left[a^{\#}(k), a^{\#}\left(k^{\prime}\right)\right]=0,
$$

where $a^{\#}$ stands for $a$ or $a^{*}$.

Let $h$ be a measurable function on $\mathbb{R}^{3}$. We define the operator $\mathrm{d} \Gamma(h)$ in $\mathcal{F}$, as follows on vectors $\psi$ in its domain:

$$
(\mathrm{d} \Gamma(h) \psi)_{n}\left(k_{1}, \ldots, k_{n}\right)=\sum_{j=1}^{n} h\left(k_{j}\right) \psi_{n}\left(k_{1}, \ldots, k_{n}\right) .
$$

The domain of $\mathrm{d} \Gamma(h)$ consists of all vectors $\psi$ such that $\mathrm{d} \Gamma(h) \psi$ is a vector in $\mathcal{F}$. We define the free-field Hamiltonian $H_{f}:=\mathrm{d} \Gamma(\omega)$, where $\omega(k):=|k|$. The Hilbert space is given by

$$
\mathcal{H}:=\mathbb{C}^{2} \otimes \mathcal{F}
$$

We define the following Hamilton operator with coupling parameter $\lambda \in \mathbb{C}$

$$
H_{\lambda}:=\tau \otimes 1+1 \otimes H_{f}+\lambda \sigma_{x} \otimes \phi(f),
$$

where

$$
\phi(f):=\int \frac{1}{\sqrt{\omega(k)}}\left(f(k) a^{*}(k)+\bar{f}(k) a(k)\right) \frac{\mathrm{d}^{3} k}{4 \pi},
$$

and

$$
\tau:=\sigma_{z}+1=\left(\begin{array}{cc}
2 & 0 \\
0 & 0
\end{array}\right), \quad \sigma_{x}=\left(\begin{array}{ll}
0 & 1 \\
1 & 0
\end{array}\right) .
$$

Throughout this paper we shall assume that $f / \sqrt{\omega} \in \mathfrak{h}$ and $f / \omega \in \mathfrak{h}$. It is well known that creation and annihilation operators are infinitesimally small with respect to the free-field Hamiltonian, see Lemma A.6 in the Appendix A. Thus, the operator $H_{\lambda}$ is a self-adjoint operator on the natural domain of $H_{0}$. The main results of this paper hold under the following hypothesis:

(H) $f \in \mathfrak{h}$ and $\|f\|_{\infty}<\infty$.

Note that $(\mathrm{H})$ implies that $f / \sqrt{\omega}$ and $f / \omega \in \mathfrak{h}$. We will use the following notation:

$$
D_{r}:=\{z \in \mathbb{C}|| z \mid<r\} .
$$

A main result of this paper is the following theorem:

Theorem 2.1. Assume $(\mathrm{H})$. There exists a $\lambda_{0}>0$ such that for all $\lambda \in D_{\lambda_{0}}, H_{\lambda}$ has an eigenvalue $E(\lambda)$ with eigenvector $\psi(\lambda)$ and eigenprojection $P(\lambda)$ satisfying,

(i) for $\lambda \in \mathbb{R} \cap D_{\lambda_{0}}, E(\lambda)=\inf \sigma\left(H_{\lambda}\right)$ and $E(\lambda)$ is non-degenerate,

(ii) $\lambda \mapsto E(\lambda)$ and $\lambda \mapsto \psi(\lambda)$ are analytic on $D_{\lambda_{0}}$,

(iii) $\lambda \mapsto P(\lambda)$ is analytic on $D_{\lambda_{0}}$ and $P(\lambda)^{*}=P(\bar{\lambda})$. 
Remark 2.2. Since we had the application to non-relativistic qed in mind, we chose Hypothesis $(\mathrm{H})$. Using a different norm for the Banach spaces we expect that one can show that the conclusion of Theorem 2.1 holds under the assumptions $\omega^{-1-\epsilon} f \in \mathfrak{h}$ and $\omega^{-1 / 2} f \in \mathfrak{h}$, for some $\epsilon>0$. Moreover, the assertion of the Theorem 2.1 without uniqueness holds if $\tau$ and $\sigma_{x}$ are replaced by hermitian $N \times N$ matrices $T$ and $A$, respectively, such that $T$ has a unique ground state and its eigenprojection $P$ satisfies $P A P=0$ and $(1-P) A(1-P)=0$.

The above result is non-trivial since the ground-state energy is not isolated from the rest of the spectrum. In that situation regular analytic perturbation theory is not applicable. We prove the existence and analyticity results of Theorem 2.1 using an operator theoretic renormalization analysis. Since that method yields the existence of a ground state but not its uniqueness, we complement the existence with the following uniqueness theorem, which we prove in the next section:

Theorem 2.3. Suppose $\lambda \in \mathbb{R}, \omega^{-1 / 2} f \in \mathfrak{h}$, and $\omega^{-1} f \in \mathfrak{h}$. Suppose $E=$ $\inf \sigma\left(H_{\lambda}\right)$ is an eigenvalue. Then $E$ is simple.

Once Theorem 2.1 has been established, one knows that the eigenvalue of $H_{\lambda}$ and the associated eigenprojection have power series expansions with nonzero radius of convergence,

$$
P(\lambda)=\sum_{n=0}^{\infty} P^{(n)} \lambda^{n}, \quad E(\lambda)=\sum_{n=0}^{\infty} E^{(n)} \lambda^{n} .
$$

It is natural to ask whether the expansion coefficients can be obtained by means of analytic perturbation theory. This is indeed the case, as we now outline. For details, see Theorem 13.1 in Sect. 13. We introduce a cutoff $\sigma \geq 0$ and define the infrared regularized Hamiltonian

$$
H_{\lambda, \sigma}:=H_{0}+\lambda T_{\sigma}
$$

with $T_{\sigma}:=\sigma_{x} \otimes \phi\left(f_{\sigma}\right)$, where $f_{\sigma}(k)=f(k)$ if $|k| \geq \sigma$ and 0 otherwise. This effectively turns the ground-state energy into an isolated eigenvalue, after a trivial part of the Hamiltonian has been factored out. In this situation regular perturbation theory becomes applicable. It is straightforward to show using analytic perturbation theory, see the proof of Theorem 13.1, that for each $\sigma>0$ there exists a $\lambda_{0}(\sigma)>0$ such that for all $\lambda \in D_{\lambda_{0}(\sigma)}$, the Hamiltonian $H_{\lambda, \sigma}$ has an eigenvalue $\widehat{E}_{\sigma}(\lambda)$ with eigenprojection $\widehat{P}_{\sigma}(\lambda)$. Furthermore, we have convergent power series expansions (see Kato's book [18])

$$
\widehat{P}_{\sigma}(\lambda)=\sum_{n=0}^{\infty} \widehat{P}_{\sigma}^{(n)} \lambda^{n}, \quad \widehat{E}_{\sigma}(\lambda)=\sum_{n=0}^{\infty} \widehat{E}_{\sigma}^{(n)} \lambda^{n} .
$$

Using analytic perturbation theory one can show that

$$
\widehat{P}_{\sigma}^{(n)}=-\sum_{\substack{\nu_{1}+\cdots+\nu_{n+1}=n, \nu_{i} \geq 0}} S_{\sigma}^{\left(\nu_{1}\right)} T_{\sigma} S_{\sigma}^{\left(\nu_{2}\right)} \cdots T_{\sigma} S_{\sigma}^{\left(\nu_{n+1}\right)}
$$


where

$$
S_{\sigma}^{(\nu)}= \begin{cases}-P_{\Omega_{\downarrow}}, & \nu=0 \\ H_{0}^{-\nu} \bar{P}_{\Omega_{\downarrow}} Q_{\sigma}, & \nu \geq 1,\end{cases}
$$

$P_{\Omega_{\downarrow}}$ denotes the orthogonal projection onto, $\Omega_{\downarrow}$, the ground state of $H_{0}$, i.e.,

$$
\Omega_{\downarrow}:=\left(\begin{array}{l}
0 \\
1
\end{array}\right) \otimes \Omega
$$

$\bar{P}_{\Omega_{\downarrow}}=1-P_{\Omega_{\downarrow}}$, and $Q_{\sigma}$ denotes the orthogonal projection in $\mathcal{F}$ onto the natural embedding of $\mathcal{F}\left(\mathfrak{h}_{\sigma}^{(+)}\right)$in $\mathcal{F}$, with $\mathfrak{h}_{\sigma}^{(+)}:=L^{2}\left(\left\{k \in \mathbb{R}^{3}|| k \mid \geq \sigma\right\}\right)$. Moreover, the coefficients of the energy expansion can be obtained using the relation

$$
\widehat{E}_{\sigma}^{(n)}=\operatorname{tr}\left(T_{\sigma} \widehat{P}_{\sigma}^{(n-1)} / n\right),
$$

which can be found in [18, p. 80, Eq. (2.34)], and is in fact easy to see. Analytic perturbation theory does not allow us to control the radius of convergence $\lambda_{0}(\sigma)$ as $\sigma$ tends to zero. That is, we cannot rule out the possibility that $\lambda_{0}(\sigma) \rightarrow 0$ in this limit. In order to control the radius of convergence of (2.5) we have to resort back to renormalization. Using a continuity argument in connection with the renormalization procedure we obtain the following theorem, which essentially states that the ground-state energy and the eigenprojection depend continuously on $\sigma$ :

Theorem 2.4. Assume $(\mathrm{H})$. There exists a $\lambda_{0}>0$ such that for all $\lambda \in D_{\lambda_{0}}$ and all $\sigma \geq 0, H_{\lambda, \sigma}$ has an eigenvalue $E_{\sigma}(\lambda)$ with eigenvector $\psi_{\sigma}(\lambda)$ and eigenprojection $P_{\sigma}(\lambda)$ satisfying (i)-(iii) of Theorem 2.1. Moreover, $E_{\sigma}(\lambda), \psi_{\sigma}(\lambda)$, and $P_{\sigma}(\lambda)$, as well as the expansion coefficients $E_{\sigma}^{(n)}$ and $P_{\sigma}^{(n)}$, in

$$
\begin{aligned}
& E_{\sigma}(\lambda)=\sum_{n=0}^{\infty} E_{\sigma}^{(2 n)} \lambda^{2 n} \\
& P_{\sigma}(\lambda)=\sum_{n=0}^{\infty} P_{\sigma}^{(n)} \lambda^{n},
\end{aligned}
$$

are continuous functions of $\sigma \in[0, \infty)$.

By the uniqueness of the ground state, we know from Theorem 2.4 and the result from perturbation theory (for details see Theorem 13.1) that for any $\sigma>0$ there exists an open ball, $D_{\lambda_{0}(\sigma)}$, such that $\widehat{P}_{\sigma}(\lambda)=P_{\sigma}(\lambda)$ and $\widehat{E}_{\sigma}(\lambda)=E_{\sigma}(\lambda)$ for all $\lambda \in D_{\lambda_{0}(\sigma)} \cap \mathbb{R}$. By analytic continuation it follows that $\widehat{P}_{\sigma}(\lambda)$ and $\widehat{E}_{\sigma}(\lambda)$ have an analytic extension to a ball, $D_{\lambda_{0}}$, which is independent of $\sigma>0$. Moreover, these extensions agree with $P_{\sigma}(\lambda)$ and $E_{\sigma}(\lambda)$ on that ball, respectively. Thus, we have shown that Theorem 2.4 implies the following corollary:

Corollary 2.5. Assume $(\mathrm{H})$. There exists a $\lambda_{0}>0$ such that for all $\sigma>0$, $\widehat{P}_{\sigma}(\lambda)$ and $\widehat{E}_{\sigma}(\lambda)$ have an analytic extension to $D_{\lambda_{0}}$, and on $D_{\lambda_{0}}$ they agree with $P_{\sigma}(\lambda)$ and $E_{\sigma}(\lambda)$. In particular, for any $\sigma>0$ we have

$$
\widehat{P}_{\sigma}^{(n)}=P_{\sigma}^{(n)}, \quad \widehat{E}_{\sigma}^{(n)}=E_{\sigma}^{(n)},
$$


and the following limits exist:

$$
\lim _{\sigma \downarrow 0} \widehat{P}_{\sigma}^{(n)}=P^{(n)}, \quad \lim _{\sigma \downarrow 0} \widehat{E}_{\sigma}^{(n)}=E^{(n)} .
$$

Note that the existence of the limit (2.11) is in view of Equation (2.6) not obvious. In particular, certain summands in that sum are divergent as $\sigma \rightarrow 0$. But the total sum must be convergent by (2.11). We note the following remark illustrating this observation (see Sect. 13):

Remark 2.6. Consider the sum (2.6). For $n \leq 3$ all terms in the sum converge as $\sigma$ tends to 0 . For $n=4$, there are terms which diverge. Let

$$
\begin{aligned}
& A_{\sigma}:=S_{\sigma}^{(1)} T_{\sigma} S_{\sigma}^{(1)} T_{\sigma} S_{\sigma}^{(1)} T_{\sigma} S_{\sigma}^{(1)} T_{\sigma} S_{\sigma}^{(0)} \\
& B_{\sigma}:=S_{\sigma}^{(2)} T_{\sigma} S_{\sigma}^{(1)} T_{\sigma} S_{\sigma}^{(0)} T_{\sigma} S_{\sigma}^{(1)} T_{\sigma} S_{\sigma}^{(0)} .
\end{aligned}
$$

Then $\lim _{\sigma \downarrow 0} A_{\sigma}$ and $\lim _{\sigma \downarrow 0} B_{\sigma}$ diverge but $\lim _{\sigma \downarrow 0}\left(A_{\sigma}+B_{\sigma}\right)$ converges.

It would be interesting to understand the nature of the cancellations occurring in the coefficients (2.6) in a systematic way. Moreover, a sufficiently good estimate on these coefficients could possibly provide an alternative way to prove Theorem 2.1.

Let us now outline the paper. In Sect. 3, we prove that a possible ground state of the spin-boson model has to be unique. We use this result to establish the equivalence of expansion coefficients obtained on the one hand by perturbation theory and on the other hand by operator theoretic renormalization.

Since Theorem 2.1 is a special case of Theorem 2.4, we only prove the latter. The proof is based on the operator theoretic renormalization analysis, as outlined in [2]. Sections $4-10$ are devoted to the renormalization analysis. In Sect. 4, we introduce the smooth Feshbach map associated with a pair of operators and we review some of its isospectrality properties, which will be needed later. In Sect. 5, we define a Banach space of integral kernels and show its bijective correspondence to a subspace of Hamiltonians acting on Fockspace. In Sect. 6, we define the renormalization transformation on the level of operators. In Sect. 7, we derive the induced action of the renormalization transformation on the space of integral kernels. In Sect. 8, we show that the renormalization transformation preserves analyticity and continuity properties of the integral kernels. In Sect. 9, we show that the renormalization transformation acts as a contraction in a subset of the Banach space of integral kernels for which the sum of the number of creation and annihilation operators is even. In Sect. 10, we construct the eigenvector and the corresponding eigenvalue of $H_{\lambda, \sigma}$. In Lemmas 10.1 and 10.2 we collect the convergence estimates which will be needed to prove Theorem 10.4, which states a general result about the analyticity (respectively continuity) of the ground state and its energy. This section contains the main results needed from the operator theoretic renormalization analysis to prove Theorem 2.4. In Sect. 11, we perform the initial two Feshbach transformations. This allows us to turn the spectral problem of the spin-boson Hamiltonian into a spectral problem of a new operator involving a sum of normal ordered monomials in creation and annihilation operators where 
for each summand the total number of creation and annihilation operators is even. Moreover, we present a basic estimate which allows us to initiate the renormalization procedure. In Sect. 12, we put all the pieces together and prove Theorem 2.4. For this we will mainly use results stated in Sects. 10 and 11.

In Sect. 13, we discuss analytic perturbation theory and Remark 2.6. In Appendix A, we collect a few basic estimates and identities involving creation and annihilation operators. In Appendix B, we discuss Wick's theorem and a generalization thereof.

\section{Uniqueness}

In this section we prove Theorem 2.3. It involves a special choice of $L^{2}$ space and a positivity argument. We first introduce the notation

$$
\Phi(f)=a^{*}(f)+a(f), \quad f \in \mathfrak{h}=L^{2}\left(\mathbb{R}^{3}\right)
$$

and prove a lemma.

Lemma 3.1. Given $\widehat{f}_{0} \in \mathfrak{h}$ then there exists a real Hilbert space $\mathfrak{k} \subset \mathfrak{h}$ with the properties

(1) $\mathfrak{k}$ is invariant under $\left\{\mathrm{e}^{-t \omega} \mid t \geq 0\right\}$.

(2) $\mathfrak{k}+i \mathfrak{k}=\mathfrak{h}$

(3) $[\Phi(f), \Phi(g)]=0$ if $f, g \in \mathfrak{k}$

(4) $\widehat{f}_{0} \in \mathfrak{k}$.

Proof. Given $f \in \mathfrak{h} \backslash\{0\}$, let $V_{f}$ be the real Hilbert space given by

$$
V_{f}=\{g(\omega) f \in \mathfrak{h} \mid g \text { a real measurable function }\}
$$

It is easy to see that $V_{f}$ is closed. We consider the family $\mathfrak{H}$ of superorthogonal sets of vectors $\left\{f_{j} \in \mathfrak{h} \mid 0 \leq j<N\right\}, N \leq \infty$, where superorthogonal means that $V_{f_{j}}$ is orthogonal to $f_{k}$ for all $k \neq j$. We order the set $\mathfrak{H}$ by inclusion. An easy application of Zorn's lemma shows there is a maximal element, $\tau=\left\{f_{j} \in \mathfrak{h} \mid 0 \leq j<N_{\tau}\right\}$, of $\mathfrak{H}$. Let us write

$$
\mathfrak{k}=\bigoplus_{j=0}^{N_{\tau}-1} V_{f_{j}},
$$

where in the direct sum we only allow linear combinations with real coefficients so that $\mathfrak{k}$ is a real Hilbert space. The properties (1) and (4) are clear while (3) follows from

$$
[\Phi(f), \Phi(g)]=2 i \operatorname{Im}(f, g)
$$

To see that $(f, g)$ is real for $f, g \in \mathfrak{k}$ note $(f, g)=0$ if $f$ and $g$ are in different $V_{f_{j}}$ 's while if $f=h_{1}(\omega) f_{j} \in \mathfrak{h}$ and $g=h_{2}(\omega) f_{j} \in \mathfrak{h}$ with $h_{1}$ and $h_{2}$ real then $(f, g)=\int h_{1}(\omega) h_{2}(\omega)\left|f_{j}(\omega)\right|^{2} \mathrm{~d}^{3} k$ is clearly real. To see $(2)$ note that if $h \in \mathfrak{h}$ is orthogonal to $\mathfrak{k}$, then by an approximation argument the same is true of all $g(\omega) h \in \mathfrak{h}$ with $g$ measurable. Thus, if $h \neq 0, \tau \cup\{h\} \in \mathfrak{H}$ and $\tau$ is not maximal. Thus, $h=0$. Let $B=\left\{v_{j} \mid j \in \mathbb{N}\right\}, v_{j} \in \mathfrak{k}$, be an orthonormal basis for $\mathfrak{k}$. Then, 
by what we have just proved, $B$ is an orthonormal basis for $\mathfrak{h}$. If $g \in \mathfrak{h}$, then $g=\sum_{j=1}^{\infty}\left(a_{j}+i b_{j}\right) v_{j}$ with $a_{j}, b_{j}$ real and $\sum_{j=1}^{\infty}\left(\left|a_{j}\right|^{2}+\left|b_{j}\right|^{2}\right)<\infty$. Then,

$$
g=\sum_{j=1}^{\infty} a_{j} v_{j}+i \sum_{j=1}^{\infty} b_{j} v_{j} \in \mathfrak{k}+i \mathfrak{k} .
$$

Proof of Theorem 2.3. From the lemma and the fact that the closure of the linear span of

$$
\left\{\mathrm{e}^{i \Phi(f)} \Omega \mid f \in \mathfrak{k}\right\}
$$

is in fact all of Fock space, the spectral theorem shows that $\mathcal{F}$ is unitarily equivalent to $L^{2}(Q, \mathrm{~d} \mu)$ for some probability measure space $(Q, \mu)$ (we suppress the $\sigma$-algebra). In this representation $\Omega$ is the function 1 and we can take all the $\Phi(f)$ 's, $f \in \mathfrak{k}$ to be real Gaussian random variables with $\Phi(f+g)=\Phi(f)+\Phi(g)$ for $f, g \in \mathfrak{k}$. Following [22], in the new representation $\mathrm{e}^{-t H_{f}}$ is a positivity preserving operator on $L^{2}(Q, \mathrm{~d} \mu)$. Let $U=u \otimes 1$, with $u=\mathrm{e}^{-i(\pi / 4) \sigma_{y}}$. Note that $u \sigma_{x} u^{-1}=\sigma_{z}, u \sigma_{z} u^{-1}=-\sigma_{x}$, and thus taking $\lambda=1$ without loss of generality,

$$
H:=U H_{1} U^{-1}=\widetilde{H}_{0}+\sigma_{z} \otimes \phi(f)
$$

where

$$
\tilde{H}_{0}=1-\sigma_{x} \otimes 1+1 \otimes H_{f} .
$$

We write $(4 \pi)^{-1} \omega^{-1 / 2} f=\widehat{f}_{0}$ so that $\phi(f)=\Phi\left(\widehat{f}_{0}\right)$. $\widetilde{H}_{0}$ has a non-degenerate ground state in $\mathbb{C}^{2} \otimes \mathcal{F}$, namely

$$
\left(\begin{array}{l}
1 \\
1
\end{array}\right) \otimes \Omega=: \Psi_{0}
$$

We note that

$$
\mathbb{C}^{2} \otimes \mathcal{F} \cong L^{2}(\{-1,1\} \times Q, \mathrm{~d} p \otimes \mathrm{d} \mu)
$$

where $p(\{1\})=p(\{-1\})=1$. In this representation, if $f \in L^{2}(\{-1,1\} \times$ $Q ; \mathrm{d} p \otimes \mathrm{d} \mu)$, then

$$
\begin{aligned}
\left(\left(\sigma_{x} \otimes 1\right) f\right)( \pm 1, \cdot) & =f(\mp 1, \cdot) \\
\left(\left(1 \otimes \mathrm{e}^{-t H_{f}}\right) f\right)(s, \cdot) & =\mathrm{e}^{-t H_{f}} f(s, \cdot) \\
\left(\left(\sigma_{z} \otimes \Phi\left(\widehat{f}_{0}\right)\right) f\right)( \pm 1, \cdot) & = \pm \Phi\left(\widehat{f}_{0}\right) f( \pm 1, \cdot) .
\end{aligned}
$$

In addition, $\mathrm{e}^{-t\left(-\sigma_{x} \otimes 1\right)}$ is positivity preserving (clear by expanding the exponential in a power series) and thus so is $\mathrm{e}^{-t \widetilde{H}_{0}}$. The operator $\widetilde{H}_{0}$ has a non-degenerate ground state given by the function 1 . A direct application of Theorem XIII.43 of [20] then implies $L^{\infty}(\{-1,1\} \times Q) \cup\left\{\mathrm{e}^{-\widetilde{H}_{0}}\right\}$ acts irreducibly in $L^{2}(\{-1,1\} \times Q)$. Since $\sigma_{z} \otimes \Phi\left(\widehat{f}_{0}\right)$ is infinitesimally $\widetilde{H}_{0}$ bounded, Theorem XIII.45 of [20] then shows $L^{\infty}(\{-1,1\} \times Q) \cup\left\{\mathrm{e}^{-H}\right\}$ acts irreducibly in $L^{2}(\{-1,1\} \times Q)$. Finally, according to Theorem XIII.43 of [20], if $E=\inf \sigma(H)$ is an eigenvalue of $H$, then it is non-degenerate. 
We end this section with a proof that $\sigma\left(H_{\lambda}\right)$ is a half line. In fact using the ideas developed in Lemma 3.1 we prove a bit more:

Proposition 3.2. Suppose $\lambda \in \mathbb{R}, \omega^{-1 / 2} f \in \mathfrak{h}$, and $\omega^{-1} f \in \mathfrak{h}$. Let $E=$ $\inf \sigma\left(H_{\lambda}\right)$. Then $\sigma_{a c}\left(H_{\lambda}\right)=[E, \infty)$.

Here, $\sigma_{a c}\left(H_{\lambda}\right)$ is the absolutely continuous spectrum of $H_{\lambda}$.

Proof. Using the notation of Lemma 3.1 and Theorem 2.3, let $\mathfrak{h}_{1}=V_{\widehat{f}_{0}}+i V_{\widehat{f}_{0}}$. Using polar coordinates $(u, t)$ where $u \in S^{2}$ and $t \in(0, \infty)$ we have $\widehat{f}_{0}(t) \in$ $L^{2}\left(S^{2}\right)$ for a.e. $t$. According to [9] the space orthogonal to $\widehat{f}_{0}(t)$ in $L^{2}\left(S^{2}\right)$ has an orthonormal basis $\left\{e_{j}(t) \mid j \in \mathbb{N}\right\}$ where the vectors $e_{j}(t)$ are measurable in the variable $t$. The space of functions $\sum_{n=1}^{\infty} g_{n}(t) e_{n}(t)$ with $g_{n} \in L^{2}\left(t^{2} d t\right)$ and $\sum_{n=1}^{\infty} \int_{0}^{\infty}\left|g_{n}(t)\right|^{2} t^{2} d t<\infty$ is exactly $\mathfrak{h}_{2}:=\mathfrak{h}_{1}^{\perp}$. Fix an orthonormal basis $\left\{\hat{e}_{j} \mid j \in \mathbb{N}\right\}$ for $L^{2}\left(S^{2}\right)$ and note that defining $u(t): \widehat{f}_{0}(t)^{\perp} \rightarrow L^{2}\left(S^{2}\right)$ by linearity and continuity from $u(t) e_{j}(t):=\hat{e}_{j}$, then $u(t)$ is unitary and $U$ given by $U g(t)=u(t) g(t)$ is a unitary map of $\mathfrak{h}_{2}$ onto $\mathfrak{h}=L^{2}\left(\mathbb{R}^{3}\right)$.

We now factor the Hilbert space $\mathcal{H}=\mathbb{C}^{2} \otimes \mathcal{F}$ as $\mathcal{H}=\mathbb{C}^{2} \otimes \mathcal{F}\left(\mathfrak{h}_{1}\right) \otimes \mathcal{F}\left(\mathfrak{h}_{2}\right)$. With respect to this factorization we write $H_{\lambda}=\tau \otimes 1 \otimes 1+1 \otimes H_{f}^{(1)} \otimes$ $1+\lambda \sigma_{x} \otimes \phi(f) \otimes 1+1 \otimes 1 \otimes H_{f}^{(2)}$ where $H_{f}^{(j)}$ is the restriction of $H_{f}$ to $\mathcal{F}\left(\mathfrak{h}_{j}\right) \cap D\left(H_{f}\right)$. We define $\tilde{H}_{\lambda}$ by the equation $H_{\lambda}=\tilde{H}_{\lambda} \otimes 1+1 \otimes H_{f}^{(2)}$. Let $\Gamma(U): \mathcal{F}\left(\mathfrak{h}_{2}\right) \rightarrow \mathcal{F}(\mathfrak{h})$ be the unitary operator satisfying $\Gamma(U) \Omega=\Omega$ and $\Gamma(U) S_{n}\left(g_{1} \otimes \cdots \otimes g_{n}\right)=S_{n}\left(U g_{1} \otimes \cdots \otimes U g_{n}\right)$. It is easy to see that $\Gamma(U) H_{f}^{(2)}=H_{f} \Gamma(U)$ so that $H_{f}^{(2)}$ restricted to $\Omega^{\perp}$ is absolutely continuous. Since clearly $E=\inf \sigma\left(\tilde{H}_{\lambda}\right)$ and the convolution of an absolutely continuous measure with another measure is absolutely continuous, the proposition easily follows.

\section{Smooth Feshbach Property}

In this section we follow $[2,13]$. We introduce the Feshbach map and state its basic isospectrality properties. This will be needed to define the renormalization transformation and to construct the ground state and the ground state energy.

Let $\chi$ and $\bar{\chi}$ be commuting, nonzero bounded operators, acting on a separable Hilbert space $\mathcal{H}$ and satisfying $\chi^{2}+\bar{\chi}^{2}=1$. A Feshbach pair $(H, T)$ for $\chi$ is a pair of closed operators with the same domain,

$$
H, T: D(H)=D(T) \subset \mathcal{H} \rightarrow \mathcal{H}
$$

such that $H, T, W:=H-T$, and the operators

$$
\begin{array}{ll}
W_{\chi}:=\chi W \chi, & W_{\bar{\chi}}:=\bar{\chi} W \bar{\chi} \\
H_{\chi}:=T+W_{\chi}, & H_{\bar{\chi}}:=T+W_{\bar{\chi}},
\end{array}
$$

defined on $D(T)$ satisfy the following assumptions: 
(a) $\chi T \subset T \chi$ and $\bar{\chi} T \subset T \bar{\chi}$,

(b) $T, H_{\bar{\chi}}: D(T) \cap \operatorname{Ran} \bar{\chi} \rightarrow \operatorname{Ran} \bar{\chi}$ are bijections with bounded inverse,

(c) $\bar{\chi} H_{\bar{\chi}}^{-1} \bar{\chi} W \chi: D(T) \subset \mathcal{H} \rightarrow \mathcal{H}$ is a bounded operator.

Remark 4.1. By abuse of notation we write $H_{\bar{\chi}}^{-1} \bar{\chi}$ for $\left(H_{\bar{\chi}} \uparrow \operatorname{Ran} \bar{\chi} \cap D(T)\right)^{-1}$ $\bar{\chi}$ and likewise $T^{-1} \bar{\chi}$ for $(T \uparrow \operatorname{Ran} \bar{\chi} \cap D(T))^{-1} \bar{\chi}$.

An operator $A: D(A) \subset \mathcal{H} \rightarrow \mathcal{H}$ is called bounded invertible in a subspace $V \subset \mathcal{H}(V$ not necessarily closed), if $A: D(A) \cap V \rightarrow V$ is a bijection with bounded inverse. Given a Feshbach pair $(H, T)$ for $\chi$, the operator

$$
F_{\chi}(H, T):=H_{\chi}-\chi W \bar{\chi} H_{\bar{\chi}}^{-1} \bar{\chi} W \chi
$$

on $D(T)$ is called the Feshbach map of $H$. The mapping $(H, T) \mapsto F_{\chi}(H, T)$ is called the Feshbach map. The auxiliary operators

$$
\begin{aligned}
& Q_{\chi}:=Q_{\chi}(H, T):=\chi-\bar{\chi} H_{\bar{\chi}}^{-1} \bar{\chi} W \chi, \\
& Q_{\chi}^{\#}:=Q_{\chi}^{\#}(H, T):=\chi-\chi W \bar{\chi} H_{\bar{\chi}}^{-1} \bar{\chi} .
\end{aligned}
$$

are by conditions (a), (c), bounded, and $Q_{\chi}$ leaves $D(T)$ invariant. The Feshbach map is isospectral in the sense of the following theorem:

Theorem 4.2. Let $(H, T)$ be a Feshbach pair for $\chi$ on a Hilbert space $\mathcal{H}$. Then the following holds:

(i) Let $V$ be subspace with $\operatorname{Ran} \chi \subset V \subset \mathcal{H}$,

$$
T: D(T) \cap V \rightarrow V, \text { and } \quad \bar{\chi} T^{-1} \bar{\chi} V \subset V .
$$

Then $H: D(H) \subset \mathcal{H} \rightarrow \mathcal{H}$ is bounded invertible if and only if $F_{\chi}(H, T)$ : $D(T) \cap V \rightarrow V$ is bounded invertible in $V$. Moreover,

$$
\begin{aligned}
H^{-1} & =Q_{\chi} F_{\chi}(H, T)^{-1} Q_{\chi}^{\#}+\bar{\chi} H_{\bar{\chi}}^{-1} \bar{\chi}, \\
F_{\chi}(H, T)^{-1} & =\chi H^{-1} \chi+\bar{\chi} T^{-1} \bar{\chi} .
\end{aligned}
$$

(ii) $\quad \chi \operatorname{ker} H \subset \operatorname{ker} F_{\chi}(H, T)$ and $Q_{\chi} \operatorname{ker} F_{\chi}(H, T) \subset \operatorname{ker} H$. The mappings

$$
\begin{aligned}
& \chi: \operatorname{ker} H \rightarrow \operatorname{ker} F_{\chi}(H, T), \\
& Q_{\chi}: \operatorname{ker} F_{\chi}(H, T) \rightarrow \operatorname{ker} H,
\end{aligned}
$$

are linear isomorphisms and inverse to each other.

The proof of Theorem 4.2 can be found in [13]. The next lemma gives sufficient conditions for two operators to be a Feshbach pair. It follows from a Neumann expansion, [13].

Lemma 4.3. Conditions (a), (b), and (c) on Feshbach pairs are satisfied if

(a') $\chi T \subset T \chi$ and $\bar{\chi} T \subset T \bar{\chi}$,

(b') $T$ is bounded invertible in $\operatorname{Ran} \bar{\chi}$,

(c') $\left\|T^{-1} \bar{\chi} W \bar{\chi}\right\|<1,\left\|\bar{\chi} W T^{-1} \bar{\chi}\right\|<1$, and $T^{-1} \bar{\chi} W \chi$ is a bounded operator.

Moreover, we need the identity given in the following Lemma. The identity follows after some manipulations of the definitions. A proof can be found for example in [13]. 
Lemma 4.4. Let $(H, T)$ be a Feshbach pair for $\chi$. Then $H Q_{\chi}=\chi F_{\chi}(H, T)$ on $D(T)$.

\section{Banach Spaces of Hamiltonians}

In this section we introduce Banach spaces of integral kernels, which parameterize certain subspaces of the space of bounded operators on Fock space. These subspaces are suitable to study an iterative application of the Feshbach map and to formulate the contraction property. We mainly follow the exposition in [2]. However, we use a different class of Banach spaces. In Equations (5.3) and (5.4) we define the parametrization. The Banach space of integral kernels which we will work with is introduced in Definition 5.1. Theorem 5.4 states that this parametrization is injective and continuous. Since our analysis will involve resolvents, the previously introduced space will be lifted in Definition 5.5 to a space of Banach space valued functions. To show continuity in the infrared cutoff, we introduce a notation of componentwise continuity in Definition 5.6, which will imply continuity of the associated operators, Lemma 5.7.

The renormalization transformation will be defined on operators acting on the reduced Fock space

$$
\mathcal{H}_{\text {red }}:=P_{\text {red }} \mathcal{F}
$$

where we introduced the notation $P_{\text {red }}:=\mathbb{1}_{[0,1]}\left(H_{f}\right)$. By $\mathbb{1}_{A}$ we will denote the characteristic function of the set $A$. We will investigate bounded operators in $\mathcal{B}\left(\mathcal{H}_{\text {red }}\right)$ of the form

$$
\begin{aligned}
H(w) & :=T[w]+W[w], \\
T[w] & :=H_{0,0}(w), \\
W[w] & :=\sum_{m+n \geq 1} H_{m, n}(w),
\end{aligned}
$$

with $H_{m, n}(w):=H_{m, n}\left(w_{m, n}\right), m, n \in \mathbb{N}_{0}$, and

$$
\begin{aligned}
& H_{m, n}\left(w_{m, n}\right) \\
& :=P_{\text {red }} \int_{B_{1}^{m+n}} \frac{\mathrm{d} K^{(m, n)}}{\left|K^{(m, n)}\right|^{1 / 2}} a^{*}\left(k^{(m)}\right) w_{m, n}\left(H_{f}, K^{(m, n)}\right) a\left(\tilde{k}^{(n)}\right) P_{\text {red }}\left\lceil\mathcal{H}_{\text {red }},\right.
\end{aligned}
$$

where $w_{m, n} \in L^{\infty}\left([0,1] \times B_{1}^{m} \times B_{1}^{n}\right)$ is an integral kernel and $w=$ $\left(w_{m, n}\right)_{(m, n) \in \mathbb{N}_{0}^{2}}$ a sequence of integral kernels. For $m=n=0$ we mean by relation (5.4) that $H_{0,0}\left(w_{0,0}\right):=w_{0,0}\left(H_{f}\right) \uparrow \mathcal{H}_{\text {red }}$. We have used and will henceforth use the following notation:

$$
\begin{aligned}
B_{1} & :=\left\{x \in \mathbb{R}^{3}|| x \mid<1\right\} \\
k^{(m)} & :=\left(k_{1}, \ldots, k_{m}\right) \in \mathbb{R}^{3 m}, \quad \widetilde{k}^{(n)}:=\left(\widetilde{k}_{1}, \ldots, \widetilde{k}_{n}\right) \in \mathbb{R}^{3 n}, \\
K^{(m, n)} & :=\left(k^{(m)}, \widetilde{k}^{(n)}\right), \quad \mathrm{d} K^{(m, n)}:=\frac{\mathrm{d} k^{(m)}}{(4 \pi)^{m}} \frac{\mathrm{d} \widetilde{k}^{(n)}}{(4 \pi)^{n}},
\end{aligned}
$$




$$
\begin{aligned}
\mathrm{d} k^{(m)} & :=\prod_{i=1}^{m} \mathrm{~d}^{3} k_{i}, \quad \mathrm{~d} \widetilde{k}^{(n)}:=\prod_{j=1}^{n} \mathrm{~d}^{3} \widetilde{k}_{j}, \\
a^{*}\left(k^{(m)}\right) & :=\prod_{i=1}^{m} a^{*}\left(k_{i}\right), \quad a\left(\widetilde{k}^{(m)}\right):=\prod_{j=1}^{m} a\left(\widetilde{k}_{j}\right) \\
\left|K^{(m, n)}\right| & :=\left|k^{(m)}\right| \cdot\left|\widetilde{k}^{(n)}\right|, \quad\left|k^{(m)}\right|:=\left|k_{1}\right| \cdots\left|k_{m}\right|, \quad\left|\tilde{k}^{(m)}\right|:=\left|\tilde{k}_{1}\right| \cdots\left|\tilde{k}_{m}\right|, \\
\Sigma\left[k^{(m)}\right] & :=\sum_{i=1}^{m}\left|k_{i}\right| .
\end{aligned}
$$

Note that (5.4) is understood in the sense of forms, i.e. for $\psi, \phi$ two vectors in $\mathcal{H}_{\text {red }}$ with finitely many particles we define,

$$
\begin{aligned}
& \left\langle\psi, H_{m, n}\left(w_{m, n}\right) \phi\right\rangle \\
& =\int_{B_{1}^{m+n}} \frac{\mathrm{d} K^{(m, n)}}{\left|K^{(m, n)}\right|^{1 / 2}}\left\langle a\left(k^{(m)}\right) P_{\mathrm{red}} \psi, w_{m, n}\left(H_{f}, K^{(m, n)}\right) a\left(\tilde{k}^{(n)}\right) P_{\mathrm{red}} \phi\right\rangle .
\end{aligned}
$$

A vector $\psi \in \mathcal{F}$ is said to have finitely many particles if only finitely many $\psi_{n}$ are nonzero. For the precise meaning of the vectors $a\left(k^{(m)}\right) P_{\text {red }} \psi$ and $a\left(\tilde{k}^{(n)}\right) P_{\text {red }} \phi$ see $(\mathrm{A} .1)$ in Appendix A. As shown in the proof of the next lemma, Lemma 5.3, the quadratic form (5.5) is bounded and thus defines a bounded operator. Note that in view of the pull-through formula, Lemma A.1, the operator in (5.4) is equal to the restriction of

$$
\begin{gathered}
\int_{B_{1}^{m+n}} \frac{\mathrm{d} K^{(m, n)}}{\left|K^{(m, n)}\right|^{1 / 2}} a^{*}\left(k^{(m)}\right) \mathbb{1}\left(H_{f}+\Sigma\left[k^{(m)}\right] \leq 1\right) \\
w_{m, n}\left(H_{f}, K^{(m, n)}\right) \mathbb{1}\left(H_{f}+\Sigma\left[\tilde{k}^{(n)}\right] \leq 1\right) a\left(\tilde{k}^{(n)}\right)
\end{gathered}
$$

to the subspace $\mathcal{H}_{\text {red }}$. Thus, we can restrict attention to integral kernels $w_{m, n}$ which are essentially supported on the set

$$
\begin{aligned}
Q_{m, n}:=\left\{\left(r, K^{(m, n)}\right) \in[0,1] \times B_{1}^{m+n} \mid r \leq 1-\max \left(\Sigma\left[k^{(m)}\right], \Sigma\left[\widetilde{k}^{(m)}\right]\right)\right\}, & \\
m+n \geq 1, & \\
Q_{0,0}:=[0,1] . &
\end{aligned}
$$

Moreover, note that integral kernels can always be assumed to be symmetric with respect to the interchange of photon coordinates. That is, they lie in the range of the symmetrization operator, which is defined as follows:

$$
\begin{aligned}
& w_{M, N}^{(\mathrm{sym})}\left(r, K^{(M, N)}\right) \\
& \quad:=\frac{1}{N ! M !} \sum_{\pi \in S_{M}} \sum_{\tilde{\pi} \in S_{N}} w_{M, N}\left(r, k_{\pi(1)}, \ldots, k_{\pi(N)}, \widetilde{k}_{\widetilde{\pi}(1)}, \ldots, \widetilde{k}_{\widetilde{\pi}(M)}\right) .
\end{aligned}
$$

The renormalization procedure will involve kernels which lie in the following Banach spaces. We shall identify the space $L^{\infty}\left(B_{1}^{m+n} ; C[0,1]\right)$ with a 
subspace of $L^{\infty}\left([0,1] \times B_{1}^{m+n}\right)$ by setting $w_{m, n}\left(r, K^{(m, n)}\right):=w_{m, n}\left(K^{(m, n)}\right)(r)$ for $w_{m, n} \in L^{\infty}\left(B_{1}^{m+n} ; C[0,1]\right)$. The norm in $L^{\infty}\left(B_{1}^{m+n} ; C[0,1]\right)$ is given by

$$
\left\|w_{m, n}\right\|_{\underline{\infty}}:=\operatorname{ess~sup}_{K^{(m, n)} \in B_{1}^{m+n}} \sup _{r \geq 0}\left|w_{m, n}\left(K^{(m, n)}\right)(r)\right| \text {. }
$$

We note that for $w \in L^{\infty}\left(B_{1}^{m+n} ; C[0,1]\right)$ we have $\|w\|_{\infty} \leq\|w\|_{\infty}$. To show the continuity of the ground state and the ground-state energy as a function of the infrared cutoff we need to introduce a coarser norm

$$
\left\|w_{m, n}\right\|_{2}:=\left[\int \frac{\mathrm{d} K^{(m, n)}}{\left|K^{(m, n)}\right|^{2}} \sup _{r \in[0,1]}\left|w_{m, n}\left(r, K^{(m, n)}\right)\right|^{2}\right]^{1 / 2} .
$$

Conditions (i) and (ii) of the following definition are needed for the injectivity property stated in Theorem 5.4, below:

Definition 5.1. We define $\mathcal{W}_{m, n}^{\#}$ to be the Banach space consisting of functions $w_{m, n} \in L^{\infty}\left(B_{1}^{m+n} ; C^{1}[0,1]\right)$ satisfying the following properties:

(i) $w_{m, n}\left(1-\mathbb{1}_{Q_{m, n}}\right)=0$

(ii) $w_{m, n}\left(\cdot, k^{(m)}, \widetilde{k}^{(n)}\right)$ is totally symmetric in the variables $k^{(m)}$ and $\widetilde{k}^{(n)}$

(iii) the following norm is finite

$$
\left\|w_{m, n}\right\|^{\#}:=\left\|w_{m, n}\right\|_{\underline{\infty}}+\left\|\partial_{r} w_{m, n}\right\|_{\underline{\infty}} .
$$

Hence, for almost all $K^{(m, n)} \in B_{1}^{m+n}$ we have $w_{m, n}\left(\cdot, K^{(m, n)}\right) \in C^{1}[0,1]$, where the derivative is denoted by $\partial_{r} w_{m, n}$. For $0<\xi<1$, we define the Banach space

$$
\mathcal{W}_{\xi}^{\#}:=\bigoplus_{(m, n) \in \mathbb{N}_{0}^{2}} \mathcal{W}_{m, n}^{\#}
$$

to consist of all sequences $w=\left(w_{m, n}\right)_{m, n \in \mathbb{N}_{0}}$ satisfying

$$
\|w\|_{\xi}^{\#}:=\sum_{(m, n) \in \mathbb{N}_{0}^{2}} \xi^{-(m+n)}\left\|w_{m, n}\right\|^{\#}<\infty .
$$

Remark 5.2. We shall also use the norm $\left\|w_{m, n}\right\|^{\#}$ for any integral kernel $w_{m, n} \in L^{\infty}\left(B_{1}^{m+n} ; C^{1}[0,1]\right)$. Note that $\left\|w_{m, n}^{(\mathrm{sym})}\right\|^{\#} \leq\left\|w_{m, n}\right\|^{\#}$.

Given $w \in \mathcal{W}_{\xi}^{\#}$, we write $w_{\geq r}$ for the vector in $\mathcal{W}_{\xi}^{\#}$ given by

$$
\left(w_{\geq r}\right)_{(m, n)}:= \begin{cases}w_{m, n}, & \text { if } m+n \geq r \\ 0, & \text { otherwise. }\end{cases}
$$

We will use the following balls to define the renormalization transformation:

$$
\mathcal{B}^{\#}(\alpha, \beta, \gamma):=\left\{w \in \mathcal{W}_{\xi}^{\#}\left|\left\|\partial_{r} w_{0,0}-1\right\|_{\infty} \leq \alpha,\right| w_{0,0}(0) \mid \leq \beta,\left\|w_{\geq 1}\right\|_{\xi}^{\#} \leq \gamma\right\} .
$$

To be able to relate the integral kernels with bounded operators we need the following lemma: 
Lemma 5.3. For $w_{m, n} \in L^{\infty}\left(B_{1}^{m} \times B_{1}^{n} ; C[0,1]\right)$ we have

$$
\left\|H_{m, n}\left(w_{m, n}\right)\right\|_{\text {op }} \leq\left\|w_{m, n}\right\|_{2} \leq \frac{\left\|w_{m, n}\right\|_{\infty}}{\sqrt{n ! m !}} .
$$

Proof. For $\psi, \phi \in \mathcal{H}_{\text {red }}$ with finitely many particles we estimate by means of the Cauchy-Schwarz inequality,

$$
\begin{aligned}
& \left|\left\langle\psi, H_{m, n}\left(w_{m, n}\right) \phi\right\rangle\right| \\
& \quad \leq \int_{B_{1}^{m+n}} \frac{\sup _{r \in[0,1]}\left|w_{m, n}\left(r, K^{(m, n)}\right)\right|}{\left|K^{(m, n)}\right|^{1 / 2}}\left\|a\left(k^{(m)}\right) \psi\right\|\left\|a\left(\widetilde{k}^{(n)}\right) \phi\right\| \mathrm{d} K^{(m, n)} \\
& \quad \leq D_{m}(\psi)^{1 / 2} D_{n}(\phi)^{1 / 2}\left[\int_{B_{1}^{m+n}} \frac{\sup _{r \in[0,1]}\left|w_{m, n}\left(r, K^{(m, n)}\right)\right|^{2}}{\left|K^{(m, n)}\right|^{2}} \mathrm{~d} K^{(m, n)}\right]^{1 / 2},
\end{aligned}
$$

where

$$
D_{m}(\psi):=\int_{B_{1}^{m}}\left|k^{(m)}\right|\left\|a\left(k^{(m)}\right) \psi\right\|^{2} \mathrm{~d} k^{(m)} .
$$

By Corollary A.4 we have

$$
D_{m}(\psi) \leq\left\|H_{f}^{m / 2} \psi\right\|^{2} \leq\|\psi\|^{2} .
$$

This yields the first inequality of (5.9). The second inequality follows from evaluating the integral

$$
\int_{S_{m, n}} \frac{\mathrm{d} K^{(m, n)}}{\left|K^{(m, n)}\right|^{2}}=\frac{1}{n ! m !}
$$

over the set $S_{m, n}:=\left\{K^{(m, n)} \in B_{1}^{m+n} \mid \Sigma\left[k^{(m)}\right] \leq 1, \Sigma\left[\widetilde{k}^{(m)}\right] \leq 1\right\}$.

For $w \in \mathcal{W}_{\xi}^{\#}$, it is easy to see using (5.9) that the infinite sum in the definition of (5.1) converges in operator norm with bound

$$
\|H(w)\|_{\text {op }} \leq\|w\|_{\xi}^{\#} .
$$

In fact using (5.9), we see that

$$
\left\|H\left(w_{\geq r}\right)\right\|_{\text {op }} \leq \xi^{r}\left\|w_{\geq r}\right\|_{\xi}^{\#}
$$

We will use the following theorem from [2]:

Theorem 5.4. The map $H: \mathcal{W}_{\xi}^{\#} \rightarrow \mathcal{B}\left(\mathcal{H}_{\text {red }}\right)$ is injective and bounded. 
Sketch of Proof. The boundedness follows from (5.11). Assume that $H(w)=0$. We want to show that this implies that $w=0$. First we show that $H(w)=0$ implies $w_{0,0}=0$. To show this pick a non-negative function $f \in C_{0}^{\infty}\left(\mathbb{R}^{3}\right)$ with $\operatorname{supp} f \subset B_{1}$ and $\int f^{2}(x) \mathrm{d}^{3} x=1$. Define $f_{\epsilon, k}(x):=\epsilon^{-3 / 2} f\left(\epsilon^{-1}(x-k)\right)$ for $k \in B_{1}$. A straightforward computation gives

$$
\begin{aligned}
& \left\langle a^{*}\left(f_{\epsilon, k}\right) \Omega, H(w) a^{*}\left(f_{\epsilon, k}\right) \Omega\right\rangle \\
& =\int_{B_{1}} f_{\epsilon, k}^{2}(x) w_{0,0}(|x|) \mathrm{d}^{3} x+\int_{B_{1}^{2}} f_{\epsilon, k}\left(x_{1}\right) w_{1,1}\left(0, x_{1}, \widetilde{x}_{1}\right) f_{\epsilon, k}\left(\widetilde{x}_{1}\right) \frac{\mathrm{d} X^{(1,1)}}{\left|X^{(1,1)}\right|^{1 / 2}} .
\end{aligned}
$$

As $\epsilon$ tends to zero, the second term on the right-hand side converges to zero, because $f_{\epsilon, k}$ converges weakly to zero in $L^{2}\left(B_{1}\right)$ and the integral operator $w_{1,1}\left(0, x_{1}, \widetilde{x}_{1}\right) /\left|X^{(1,1)}\right|^{1 / 2}$ is compact. The first term converges in this limit to $w_{0,0}(|k|)$. Since by assumption $H(w)=0$, this implies $w_{0,0}=0$. To show that for $m+n \geq 0$ also $w_{m, n}$ has to be zero we proceed by induction. We prove that $w_{m, n}=0$ for all $m+n \leq l-1$ implies that $w_{m, n}=0$ for $m+n=l$. Thus fix $(\widehat{m}, \widehat{n})$ with $\widehat{m}+\widehat{n}=l$. Let $g_{1}, \ldots, g_{\widehat{m}}, h_{1}, \ldots, h_{\widehat{n}} \in L^{2}\left(B_{1}\right)$ and set

$$
\begin{gathered}
\psi=a^{*}\left(g_{1}\right) \cdots a^{*}\left(g_{\widehat{m}}\right) a^{*}\left(f_{\epsilon, k}\right) \Omega, \quad \phi=a^{*}\left(h_{1}\right) \cdots a^{*}\left(h_{\widehat{n}}\right) a^{*}\left(f_{\epsilon, k}\right) \Omega . \\
\langle\psi, H(w) \phi\rangle=\left\langle\psi, H_{\widehat{m}, \widehat{n}} \phi\right\rangle+\left\langle\psi, H_{\widehat{m}+1, \widehat{n}+1} \phi\right\rangle,
\end{gathered}
$$

where we used that by the induction hypothesis $w_{m, n}=0$ if $m+n \leq l-1$. As $\epsilon$ tends to zero, the second term on the right-hand side converges to zero, because $w-\lim _{\epsilon \downarrow 0} f_{\epsilon, k}=0$ in $L^{2}\left(B_{1}\right)$. The first term on the right-hand side converges in this limit to $(\widehat{m}+1) !(\widehat{n}+1)$ ! times

$$
\int_{B_{1}^{m+\widehat{n}}} \frac{\mathrm{d} X^{(\widehat{m}, \widehat{n})}}{\mid X^{(\widehat{m}, \widehat{n}) \mid 1 / 2}} \bar{g}_{1}\left(x_{1}\right) \cdots \bar{g}_{\widehat{m}}\left(x_{\widehat{m}}\right) w_{\widehat{m}, \widehat{n}}\left(|k|, X^{(\widehat{m}, \widehat{n})}\right) h_{1}\left(\widetilde{x}_{1}\right) \cdots h_{\widehat{n}}\left(\widetilde{x}_{\widehat{n}}\right),
$$

other contributions to $\left\langle\psi, H_{\widehat{m}, \widehat{n}} \phi\right\rangle$ vanish in this limit, again because $w-$ $\lim _{\epsilon \downarrow 0} f_{\epsilon, k}=0$ in $L^{2}\left(B_{1}\right)$. Since $H(w)=0$ and the choice of the functions $h_{i}$ and $g_{i}$ and the choice of $k \in B_{1}$ was arbitrary, we conclude that $w_{\widehat{m}, \widehat{n}}=0$. This shows Theorem 5.4.

The renormalization transformation will be defined on kernels which depend on a spectral parameter. To account for that, we introduce the following Banach space:

Definition 5.5. Let $\mathcal{W}_{\xi}$ denote the Banach space consisting of strongly analytic functions on $D_{1 / 2}$ with values in $\mathcal{W}_{\xi}^{\#}$ and norm given by

$$
\|w(\cdot)\|_{\xi}:=\sup _{z \in D_{1 / 2}}\|w(z)\|_{\xi}^{\#}
$$

For $w \in \mathcal{W}_{\xi}$ we will use the notation $w_{m, n}(z, \cdot):=w(z)_{m, n}(\cdot)$. We extend the definition of $H$ to a map from $\mathcal{W}_{\xi}$ to the strongly analytic functions on $D_{1 / 2}$ with values in $\mathcal{B}\left(H_{\text {red }}\right)$ : for $w \in \mathcal{W}_{\xi}$, we set

$$
H(w):=H \circ w,
$$


that is $(H(w))(z):=H(w(z))$ for all $z \in D_{1 / 2}$, and likewise for $H_{m, n}(\cdot)$ and $W[\cdot]$. The renormalization transformation will be defined on the following balls in $\mathcal{W}_{\xi}$ :

$$
\begin{array}{r}
\mathcal{B}(\alpha, \beta, \gamma):=\left\{w \in \mathcal{W}_{\xi} \mid \sup _{z \in D_{1 / 2}}\left\|\partial_{r} w_{0,0}(z)-1\right\|_{\infty} \leq \alpha\right. \\
\left.\sup _{z \in D_{1 / 2}}\left|w_{0,0}(z, 0)+z\right| \leq \beta,\left\|w_{\geq 1}\right\|_{\xi} \leq \gamma\right\} .
\end{array}
$$

Note that this set defines a basis of neighborhoods of the point $w^{*}$ satisfying $H\left(w^{*}(z)\right)=H_{f}-z$, i.e., $w^{*}{ }_{0,0}(z, r)=r-z$ and $w_{\geq 1}^{*}=0$, since

$$
\left\{w \in \mathcal{W}_{\xi} \mid\left\|w-w^{*}\right\|_{\xi} \leq \epsilon\right\} \subset \mathcal{B}(\epsilon, \epsilon, \epsilon) \subset\left\{w \in \mathcal{W}_{\xi} \mid\left\|w-w^{*}\right\|_{\xi} \leq 4 \epsilon\right\} .
$$

To state the contraction property of the renormalization transformation in Sect. 9, we will need to introduce the balls of even integral kernels

$$
\mathcal{B}_{0}(\alpha, \beta, \gamma):=\left\{w \in \mathcal{B}(\alpha, \beta, \gamma) \mid w_{m, n}=0 \quad \text { if } \quad m+n=\text { odd }\right\} .
$$

We say that a kernel $w \in \mathcal{W}_{\xi}$ is symmetric if $w_{m, n}(\bar{z})=\overline{w_{n, m}(z)}$. Note that because of Theorem 5.4 we have for $w \in \mathcal{W}_{\xi}$,

$$
w \text { is symmetric }: \Leftrightarrow H(w(\bar{z}))=H(w(z))^{*} .
$$

To obtain the continuity of the ground state and the ground state energy as a function of the infrared cutoff we introduce the following notion of convergence.

Definition 5.6. Let $S$ be topological space. We say that the mapping $w: S \rightarrow$ $\mathcal{W}_{\xi}^{\#}$ is componentwise $L^{2}$-continuous (c-continuous) if for all $m, n \in \mathbb{N}_{0}$ the map $s \mapsto w(s)_{m, n}$ is continuous with respect to $\|\cdot\|_{2}$, that is

$$
\lim _{s \in S, s \rightarrow s_{0}}\left\|w\left(s_{0}\right)_{m, n}-w(s)_{m, n}\right\|_{2}=0
$$

for all $s, s_{0} \in S$.

The above notion of continuity for integral kernels yields continuity of the associated operators with respect to the norm topology. This is the content of the following lemma:

Lemma 5.7. Let $w: S \rightarrow \mathcal{W}_{\xi}^{\#}$ be c-continuous and uniformly bounded, i.e., $\sup _{s \in S}\|w(s)\|_{\xi}^{\#}<\infty$. Then $H(w(\cdot)): S \rightarrow \mathcal{B}\left(\mathcal{H}_{\text {red }}\right)$ is continuous, with respect to the norm topology.

Proof. From Lemma 5.3 it follows that $H_{m, n}(w(s)) \stackrel{\|\cdot\|_{\text {op }}}{\longrightarrow} H_{m, n}\left(w\left(s_{0}\right)\right)$ as $s$ tends to $s_{0}$. The lemma now follows from a simple argument using the estimate $(5.12)$ and the uniform bound on $w(\cdot)$. 


\section{Renormalization Transformation: Definition}

In this section we define the renormalization transformation as in [2]. It is a combination of the Feshbach transformation which cuts out higher boson energies, a rescaling of the resulting operator so that it acts on the fixed subspace $\mathcal{H}_{\text {red }}$ and a conformal transformation of the spectral parameter.

Let $0<\xi<1$ and $0<\rho<1$. For $w \in \mathcal{W}_{\xi}$ we define the analytic function

$$
E_{\rho}[w](z):=\rho^{-1} E[w](z):=-\rho^{-1} w_{0,0}(z, 0)=-\rho^{-1}\langle\Omega, H(w(z)) \Omega\rangle
$$

and the set

$$
U[w]:=\left\{z \in D_{1 / 2}|| E[w](z) \mid<\rho / 2\right\} .
$$

Lemma 6.1. Let $0<\rho \leq 1 / 2$. Then, for all $w \in \mathcal{B}(\cdot, \rho / 8, \cdot)$, we have

$$
D_{3 \rho / 8} \subset U[w] \subset D_{5 \rho / 8},
$$

$\left|\partial_{z} E[w](z)-1\right| \leq 4 \rho(4-5 \rho)^{-2} \leq 8 / 9$ for all $z \in U[w]$, and $E_{\rho}[w]: U[w] \rightarrow$ $D_{1 / 2}$ is an analytic bijection.

The lemma follows directly from the following lemma by choosing the appropriate values for the corresponding constants: $(r=\rho / 2, \epsilon=\rho / 8)$.

Lemma 6.2. Let $0<\epsilon<1 / 2$, and let $E: D_{1 / 2} \rightarrow \mathbb{C}$ be an analytic function which satisfies

$$
\sup _{z \in D_{1 / 2}}|E(z)-z| \leq \epsilon
$$

Then for any $r>0$ with $r+\epsilon<1 / 2$ the following is true:

(a) For $w \in D_{r}$ there exists a unique $z \in D_{1 / 2}$ such that $E(z)=w$.

(b) The map $E: U_{r}:=\left\{z \in D_{1 / 2}|| E(z) \mid<r\right\} \rightarrow D_{r}$ is biholomorphic.

(c) We have $D_{r-\epsilon} \subset U_{r} \subset D_{r+\epsilon}$.

(d) If $z \in D_{r+\epsilon}$, then $\left|\partial_{z} E(z)-1\right| \leq \frac{\epsilon}{2}(1 / 2-(r+\epsilon))^{-2}$.

Proof. (a) Existence: For $z \in \partial D_{\epsilon+r}$ and $w \in D_{r}$,

$$
|E(z)-z| \leq \epsilon<|z|-|w| \leq|z-w|
$$

By Rouché's theorem, for any $w \in D_{r}$ there exists a unique $z \in D_{\epsilon+r}$ such that $E(z)=w$. Uniqueness: If $w \in D_{r}, z \in D_{1 / 2}$, and $E(z)=w$, then

$$
|z| \leq|E(z)|+\epsilon<r+\epsilon
$$

(b) This follows from (a) by the inverse function theorem of complex analysis.

(c) The first inclusion follows from $|E(z)| \leq|z|+\epsilon$. The second from (6.1). 
To obtain the estimate in (d), we use Cauchy's integral formula

$$
\left|\partial_{z}(E(z)-z)\right| \leq \liminf _{\eta \downarrow 0}\left|\frac{1}{2 \pi i} \int_{\partial D_{1 / 2-\eta}} \frac{E(w)-w}{(w-z)^{2}} \mathrm{~d} w\right| \leq \frac{\pi}{2 \pi} \frac{\epsilon}{(1 / 2-(r+\epsilon))^{2}} .
$$

Let $\chi_{1}$ and $\bar{\chi}_{1}$ be two functions in $C^{\infty}([0, \infty) ;[0,1])$ with $\chi_{1}^{2}+\bar{\chi}_{1}^{2}=1$, $\chi_{1}=1$ on $[0,3 / 4)$, and $\operatorname{supp} \chi_{1} \subset[0,1]$. We set

$$
\chi_{\rho}(\cdot)=\chi_{1}(\cdot / \rho), \quad \bar{\chi}_{\rho}(\cdot)=\bar{\chi}_{1}(\cdot / \rho),
$$

and use the abbreviation $\chi_{\rho}=\chi_{\rho}\left(H_{f}\right)$ and $\bar{\chi}_{\rho}=\bar{\chi}_{\rho}\left(H_{f}\right)$. It should be clear from the context whether $\chi_{\rho}$ or $\bar{\chi}_{\rho}$ denotes a function or an operator. For an explicit choice of $\chi_{1}$ and $\bar{\chi}_{1}$ see [2]. The following lemma will be needed to be able to define the Feshbach map which will be used later:

Lemma 6.3. Let $0<\rho \leq 1 / 2$. Then for all $w \in \mathcal{B}^{\#}(\rho / 8, \rho / 2, \rho / 8)$ we have

$$
\begin{gathered}
\|\left(H_{0,0}(w)\left\lceil\operatorname{Ran} \bar{\chi}_{\rho}\right)^{-1} \| \leq \frac{16}{3 \rho}\right. \\
\left\|H_{0,0}(w)^{-1} \bar{\chi}_{\rho} W[w]\right\|<\frac{2}{3}, \quad\left\|W[w] H_{0,0}(w)^{-1} \bar{\chi}_{\rho}\right\|<\frac{2}{3} .
\end{gathered}
$$

In particular $\left(H(w), H_{0,0}(w)\right)$ is a Feshbach pair for $\chi_{\rho}$.

Proof. To prove the lemma we verify the assumptions of Lemma 4.3. Clearly $\chi_{\rho}$ commutes with $H_{0,0}(w)$. For $r \in\left[\frac{3}{4} \rho, 1\right]$, we estimate

$$
\begin{aligned}
\left|w_{0,0}(r)\right| & \geq r-\left|r-\left(w_{0,0}(r)-w_{0,0}(0)\right)\right|-\left|w_{0,0}(0)\right| \\
& \geq r-r \rho / 8-\rho / 2 \geq \frac{3}{4} \rho(1-\rho / 8)-\rho / 2 \geq \frac{3 \rho}{16} .
\end{aligned}
$$

This implies that $H_{0,0}(w)$ is invertible on the range of $\bar{\chi}_{\rho}$ and that (6.2) holds. By this and $\|W[w]\| \leq \rho / 8$, which follows from (5.12), inequalities (6.3) follow. The Feshbach property now follows from Lemma 4.3 , since $\left\|\bar{\chi}_{1}\right\|_{\infty},\left\|\chi_{1}\right\|_{\infty} \leq 1$.

Remark 6.4. Note that $w \in \mathcal{B}(\alpha, \beta, \gamma)$ and $z \in U[w]$ imply $w(z) \in$ $\mathcal{B}^{\#}(\alpha, \rho / 2, \gamma)$.

In the definition of the renormalization transformation there is a scaling transformation $S_{\rho}$ which scales the energy value $\rho$ to the value 1 . It is defined as follows: For operators $A \in \mathcal{B}(\mathcal{F})$ set

$$
S_{\rho}(A)=\rho^{-1} \Gamma_{\rho} A \Gamma_{\rho}^{*},
$$

where $\Gamma_{\rho}$ is the unitary dilation on $\mathcal{F}$ which is uniquely determined by $\Gamma_{\rho} \Omega=\Omega$ and $\Gamma_{\rho} a^{\#}(k) \Gamma_{\rho}^{*}=\rho^{-3 / 2} a^{\#}\left(\rho^{-1} k\right)$, for all $k \in \mathbb{R}^{3}$. It is easy to check that $\Gamma_{\rho} H_{f} \Gamma_{\rho}^{*}=\rho H_{f}$ and hence $\Gamma_{\rho} \chi_{\rho} \Gamma_{\rho}^{*}=\chi_{1}$. We are now ready to precisely define the renormalization transformation, which in view of Lemmas 6.1 and 6.3, Remark 6.4, and Theorem 5.4 is well defined. 
Definition 6.5. Let $0<\rho \leq 1 / 2$. On $H\left(\mathcal{B}^{\#}(\rho / 8, \rho / 2, \rho / 8)\right)$ we define

$$
R_{\rho}^{\#}(H(w)):=S_{\rho}\left(F_{\chi_{\rho}}\left(H(w), H_{0,0}(w)\right)\right) \uparrow \mathcal{H}_{\text {red }}, \quad \forall w \in \mathcal{B}^{\#}(\rho / 8, \rho / 2, \rho / 8) .
$$

On $H(\mathcal{B}(\rho / 8, \rho / 8, \rho / 8))$ we define the renormalization transformation

$$
\left(R_{\rho}(H(w))\right)(z):=R_{\rho}^{\#}\left(H\left(w\left(E_{\rho}[w]^{-1}(z)\right)\right),\right.
$$

where $w \in \mathcal{B}(\rho / 8, \rho / 8, \rho / 8)$ and $z \in D_{1 / 2}$.

In view of the Feshbach property, Theorem 4.2 (ii), and since Ran $\chi_{1} \subset$ $\mathcal{H}_{\text {red }}$, it will turn out to be sufficient to study the restriction of the Feshbach map to $\mathcal{H}_{\text {red }}$.

\section{Renormalization Transformation: Kernels}

We have defined the renormalization transformation on the level of operators. In this section we will describe the induced transformation on the integral kernels. This transformation is derived the same way as in [2]. However, we use modified estimates to show that the renormalized kernel is again an element of $\mathcal{W}_{\xi}^{\#}$.

Throughout this section we assume $w \in \mathcal{B}^{\#}(\rho / 8, \rho / 2, \rho / 8)$ and $0<\rho \leq$ $1 / 2$. We will show that under suitable conditions there exists an integral kernel $\mathcal{R}_{\rho}^{\#}(w) \in \mathcal{W}_{\xi}^{\#}$, given in (7.4) below, such that

$$
R_{\rho}^{\#}(H(w))=H\left(\mathcal{R}_{\rho}^{\#}(w)\right) .
$$

This is the content of Theorem 7.4, below. Note that the uniqueness of the integral kernel will follow from Theorem 5.4. Next we show its formal existence. First, we expand the Feshbach operator into a Neumann series which is justified by Lemma 6.3 and rearrange the factorization to arrive at the following identity which holds on $\mathcal{H}_{\text {red }}$ :

$$
\begin{aligned}
F_{\chi_{\rho}}\left(H(w), H_{0,0}(w)\right) & =T+\chi_{\rho} W \chi_{\rho}-\chi_{\rho} W \bar{\chi}_{\rho}\left(T+\bar{\chi}_{\rho} W \bar{\chi}_{\rho}\right)^{-1} \bar{\chi}_{\rho} W \chi_{\rho} \\
& =T+\sum_{L=1}^{\infty}(-1)^{L-1} \chi_{\rho} W\left[\frac{\bar{\chi}_{\rho}^{2}}{T} W\right]^{L-1} \chi_{\rho},
\end{aligned}
$$

where here we used the abbreviations $W=W[w]$ and $T=T[w]$. Using the commutation relation of the creation and annihilation operators and the pullthrough formula, we bring this expression into normal order. To this end we introduce

$$
\begin{aligned}
& W_{p, q}^{m, n}[w]\left(r, K^{(m, n)}\right):=P_{\text {red }} \\
& \quad \times \int_{B_{1}^{p+q}} \frac{\mathrm{d} X^{(p, q)}}{\left|X^{(p, q)}\right|^{1 / 2}} a^{*}\left(x^{(p)}\right) w_{p+m, q+n}\left(H_{f}+r, x^{(p)}, k^{(m)}, \widetilde{x}^{(q)}, \widetilde{k}^{(n)}\right) a\left(\widetilde{x}^{(q)}\right) P_{\text {red }}
\end{aligned}
$$

which defines an operator for a.e. $K^{(m, n)} \in B_{1}^{m+n}$. In the case $m=n=0$ we set $W_{p, q}^{0,0}[w](r):=W_{p, q}[w](r)$. For later use we state an inequality in the following lemma. The inequality is obtained the same way as (5.9). 
Lemma 7.1. Let $w \in \mathcal{W}_{\xi}^{\#}$. Then

$$
\begin{aligned}
\left\|W_{p, q}^{m, n}[w]\left(r, K^{(m, n)}\right)\right\|_{\text {op }} & \leq \frac{\left\|w_{p+m, q+n}\right\|_{\infty}}{\sqrt{p ! q !}} \\
\left\|\partial_{r} W_{p, q}^{m, n}[w]\left(r, K^{(m, n)}\right)\right\|_{\text {op }} & \leq \frac{\left\|\partial_{r} w_{p+m, q+n}\right\|_{\infty}}{\sqrt{p ! q !}},
\end{aligned}
$$

where the partial derivative $\partial_{r} W_{p, q}^{m, n}[w]$ is taken in the weak operator topology.

The next theorem, [2], is a variant of Wick's Theorem and will be used to write the $L$-th summand in (7.1) in terms of integral kernels. Its proof can be found in Appendix B.

Theorem 7.2. Let $w \in \mathcal{W}_{\xi}^{\#}$ and let $F_{0}, F_{1}, \ldots, F_{L}$ be bounded Borel measurable functions on $[0, \infty)$. Then

$$
F_{0}\left(H_{f}\right) W[w] F_{1}\left(H_{f}\right) W[w] \cdots W[w] F_{L-1}\left(H_{f}\right) W[w] F_{L}\left(H_{f}\right)=H\left(\widetilde{w}^{(\mathrm{sym})}\right),
$$

where

$$
\begin{aligned}
& \widetilde{w}_{M, N}\left(r, K^{(M, N)}\right) \\
& =\sum_{\substack{m_{1}+\cdots m_{L}=M \\
n_{1}+\cdots n_{L}=N}} \sum_{\substack{p_{1}, q_{1}, \ldots, p_{L}, q_{L}: \\
m_{l}+p_{l}+n_{l}+q_{l} \geq 1}} \prod_{l=1}^{L}\left\{\left(\begin{array}{c}
m_{l}+p_{l} \\
p_{l}
\end{array}\right)\left(\begin{array}{c}
n_{l}+q_{l} \\
q_{l}
\end{array}\right)\right\} \\
& \times F_{0}\left(r+\tilde{r}_{0}\right)\left\langle\Omega, \prod_{l=1}^{L-1}\left\{W_{p_{l}, q_{l}}^{m_{l}, n_{l}}[w]\left(r+r_{l}, K_{l}^{\left(m_{l}, n_{l}\right)}\right) F_{l}\left(H_{f}+r+\widetilde{r}_{l}\right)\right\}\right. \\
& \left.W_{p_{L}, q_{L}}^{m_{L}, n_{L}}[w]\left(r+r_{L}, K_{L}^{\left(m_{L}, n_{L}\right)}\right) \Omega\right\rangle F_{L}\left(r+\widetilde{r}_{L}\right),
\end{aligned}
$$

with

$$
\begin{aligned}
K^{(M, N)} & :=\left(K_{1}^{\left(m_{1}, n_{1}\right)}, \ldots, K_{L}^{\left(m_{L}, n_{L}\right)}\right), \quad K_{l}^{\left(m_{l}, n_{l}\right)}:=\left(k_{l}^{\left(m_{l}\right)}, \widetilde{k}_{l}^{\left(n_{l}\right)}\right), \\
r_{l} & :=\Sigma\left[\widetilde{k}_{1}^{\left(n_{1}\right)}\right]+\cdots+\Sigma\left[\widetilde{k}_{l-1}^{\left(n_{l-1}\right)}\right]+\Sigma\left[k_{l+1}^{\left(m_{l+1}\right)}\right]+\cdots+\Sigma\left[k_{L}^{\left(m_{L}\right)}\right], \\
\widetilde{r}_{l} & :=\Sigma\left[\widetilde{k}_{1}^{\left(n_{1}\right)}\right]+\cdots+\Sigma\left[\widetilde{k}_{l}^{\left(n_{l}\right)}\right]+\Sigma\left[k_{l+1}^{\left(m_{l+1}\right)}\right]+\cdots+\Sigma\left[k_{L}^{\left(m_{L}\right)}\right]
\end{aligned}
$$

We use the standard convention that $\prod_{j=1}^{n} a_{j}=a_{1} a_{2} \cdots a_{n}$.

Remark 7.3. If $F_{0}, F_{L} \in C^{\infty}[0, \infty)$ have support contained in $[0,1]$, then the summands occurring in (7.2) satisfy property (i) of the definition of $\mathcal{W}_{m, n}^{\#}$ (see Definition 5.1). Because of property (i) of the definition of $\mathcal{W}_{m, n}^{\#}$, only the values of $F_{1}, \ldots, F_{L-1}$ on $[0,1]$ matter in (7.2). The supremum norm can be estimated using

$$
\left|\left\langle\Omega, A_{1} A_{2} \cdots A_{n} \Omega\right\rangle\right| \leq\left\|A_{1}\right\|_{\text {op }}\left\|A_{2}\right\|_{\text {op }} \cdots\left\|A_{n}\right\|_{\text {op }}
$$

and Lemma (7.1). Now suppose $F_{1}, \ldots, F_{L-1} \in C^{1}[0,1]$. Then, by the defining property of $\mathcal{W}_{m, n}^{\#}$ we can calculate the derivative with respect to $r$ of each summand using the Leibniz rule, where the interchange of integration and differentiation is justified by (iii) of the definition of $\mathcal{W}_{m, n}^{\#}$. Using again (7.3) and Lemma 7.1 it can be shown that each summand of $(7.2)$ is in $C^{1}[0,1]$ a.e. 
It can be shown that the involved sums converge absolutely in the $\|\cdot\|_{\xi}^{\#}$ norm. But for the moment we are only interested in the combinatorics. Using Theorem 7.2 to write expression (7.1) in terms of an operator involving integral kernels, it turns out to useful to introduce the multi-indices $\underline{m}=\left(m_{1}, \ldots, m_{L}\right) \in \mathbb{N}_{0}^{L}$, for $L \in \mathbb{N}$. We set $|\underline{m}|=m_{1}+\cdots+m_{L}$, and $\underline{0}:=(0,0, \ldots, 0) \in \mathbb{N}_{0}^{L}$. As a final step we have to scale the operator or equivalently the integral kernels. The integral kernels scale as follows: For $(m, n) \in \mathbb{N}_{0}^{2}$

$$
s_{\rho}(w)_{m, n}\left(r, K^{(m, n)}\right):=\rho^{m+n-1} w_{m, n}\left(\rho r, \rho K^{(m, n)}\right),
$$

since then

$$
P_{\text {red }} S_{\rho}(H(w)) P_{\text {red }} \uparrow \mathcal{H}_{\text {red }}=H\left(s_{\rho}(w)\right) .
$$

Following the outlined procedure above, we arrive at the renormalized integral kernels

$$
\mathcal{R}_{\rho}^{\#}(w):=\widehat{w}^{(\mathrm{sym})},
$$

where the kernels $\widehat{w}$ are given as follows: For $M+N \geq 1$,

$$
\begin{aligned}
& \widehat{w}_{M, N}\left(r, K^{(M, N)}\right):=\sum_{L=1}^{\infty}(-1)^{L-1} \rho^{M+N-1} \sum_{(\underline{m}, \underline{p}, \underline{n}, \underline{q}) \in \mathbb{N}_{0}^{4 L}:} \\
& \begin{array}{l}
(\underline{m}, \underline{p}, \underline{n}, \underline{q}) \in \mathbb{N}_{0}^{4}: \\
|\underline{m}|=M,|\underline{n}|=N,
\end{array} \\
& m_{l}+p_{l}+n_{l}+q_{l} \geq 1
\end{aligned}
$$

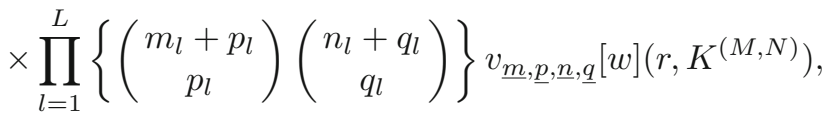

and

$$
\widehat{w}_{0,0}(r):=\rho^{-1} w_{0,0}(\rho r)+\rho^{-1} \sum_{L=2}^{\infty}(-1)^{L-1} \sum_{\substack{\underline{p}, \underline{q}) \in \mathbb{N}_{0}^{2 L}: \\ p_{l}+q_{l} \geq 1}} v_{\underline{0}, \underline{p}, \underline{0}, \underline{q}}[w](r) .
$$

Moreover, for $(\underline{m}, \underline{p}, \underline{n}, \underline{q}) \in \mathbb{N}_{0}^{4 L}$ we have introduced the expressions

$$
\begin{aligned}
& v_{\underline{m}, \underline{p}, \underline{n}, \underline{q}}[w]\left(r, K^{(|\underline{m}|,|\underline{n}|))}:=\left\langle\Omega, F_{0}[w]\left(H_{f}+\rho\left(r+\widetilde{r}_{0}\right)\right)\right.\right. \\
& \left.\quad \times \prod_{l=1}^{L}\left\{W_{p_{l}, q_{l}}^{m_{l}, n_{l}}[w]\left(\rho\left(r+r_{l}\right), \rho K_{l}^{\left(m_{l}, n_{l}\right)}\right) F_{l}[w]\left(H_{f}+\rho\left(r+\widetilde{r}_{l}\right)\right)\right\} \Omega\right\rangle,
\end{aligned}
$$

where $F_{0}[w](r):=\chi_{\rho}(r)$ and $F_{L}[w](r):=\chi_{\rho}(r)$, and for $l=1, \ldots, L-1$

$$
F_{l}[w](r):=F[w](r):=\frac{\bar{\chi}_{\rho}^{2}(r)}{w_{0,0}(r)} .
$$

Above we have used notation introduced in Theorem 7.2. From the previous discussion in this section, Theorem 7.4, below, follows apart from the property 
that the renormalized kernel is indeed an element of the Banach space $\mathcal{W}_{\xi}^{\#}$ and satisfies a uniform bound.

Theorem 7.4. Let $0<\rho \leq 1 / 2$ and $0<\xi \leq 1 / 2$ and assume $w \in \mathcal{B}^{\#}(\rho / 8, \rho / 2, \rho / 8)$. Then, $\mathcal{R}_{\rho}^{\#}(w) \in \mathcal{W}_{\xi}^{\#}$ and

$$
R_{\rho}^{\#}(H(w))=H\left(\mathcal{R}_{\rho}^{\#}(w)\right) .
$$

Moreover, $\sup _{w \in \mathcal{B}}(\rho / 8, \rho / 2, \rho / 8)\left\|\mathcal{R}_{\rho}^{\#}(w)\right\|_{\xi}^{\#}<\infty$.

The remaining part of this section concerns the proof of Theorem 7.4. To prove it we need an estimate on the kernels (7.7). Note that in view of Remark 7.3 the kernels (7.7) as well as their derivatives are well defined and can be shown to be bounded.

Lemma 7.5. Let $0<\rho \leq 1 / 2$ and $w \in \mathcal{B}^{\#}(\rho / 8, \rho / 2, \rho / 8)$. Then, for $(\underline{m}, \underline{p}, \underline{n}, \underline{q}) \in \mathbb{N}_{0}^{4 L}$ we have

$$
\left\|v_{\underline{m}, \underline{p}, \underline{n}, \underline{q}}[w]\right\|^{\#} \leq C_{L}\left(\frac{1}{t}\right)^{L-1} \prod_{l=1}^{L} \frac{\left\|w_{m_{l}+p_{l}, n_{l}+q_{l}}\right\|^{\#}}{\sqrt{p_{l} ! q_{l} !}},
$$

where

$$
t:=3 \rho / 16, \quad C_{L}:=1+2 L\left\|\partial_{r} \chi_{1}\right\|_{\infty}+(L-1) 8 .
$$

Proof. To arrive at (7.8) we start with the following estimates: For $l=0, L$ we have

$$
\begin{aligned}
\left\|F_{l}[w]\left(H_{f}+\rho\left(r+\widetilde{r}_{l}\right)\right)\right\|_{\text {op }} & \leq 1 \\
\left\|\partial_{r}\left(F_{l}[w]\left(H_{f}+\rho\left(r+\widetilde{r}_{l}\right)\right)\right)\right\|_{\text {op }} & \leq\left\|\partial_{r} \chi_{1}\right\|_{\infty} .
\end{aligned}
$$

Using (6.4) we find

$$
\|F[w]\|_{\infty} \leq\left[\inf _{r \in\left[\rho \frac{3}{4}, 1\right]}\left|w_{0,0}(r)\right|\right]^{-1} \leq \frac{1}{t}
$$

We also need an estimate on the derivative of $F[w]$,

$$
\partial_{r} F[w](r)=\rho^{-1} \frac{2 \bar{\chi}_{\rho}(r)\left[\partial_{r} \bar{\chi}_{1}\right](r / \rho)}{w_{0,0}(r)}-\frac{\bar{\chi}_{\rho}^{2}(r)\left[\partial_{r} w_{0,0}\right](r)}{\left(w_{0,0}(r)\right)^{2}} .
$$

Using (7.12) and $\bar{\chi}_{1} \partial \bar{\chi}_{1}=-\chi_{1} \partial \chi_{1}$ we estimate (7.13) and obtain

$$
\left\|\partial_{r} F[w]\right\|_{\infty} \leq \rho^{-1} \frac{2\left\|\partial_{r} \chi_{1}\right\|_{\infty}}{t}+\frac{3 / 2}{t^{2}}
$$

noting that $\left\|\partial_{r} w_{0,0}\right\|_{\infty} \leq 1+\rho / 8 \leq 3 / 2$. Next we use (7.3) and Lemma 7.1 to obtain the following estimate

$$
\left|v_{\underline{m}, \underline{p}, \underline{n}, \underline{q}}[w]\left(r, K^{(|\underline{m}|,|\underline{n}|)}\right)\right| \leq\|F[w]\|_{\infty}^{L-1} \prod_{l=1}^{L} \frac{\left\|w_{m_{l}+p_{l}, n_{l}+q_{l}}\right\|_{\infty}}{\sqrt{p_{l} ! q_{l} !}} .
$$


Using Leibniz' rule a similar estimate yields,

$$
\begin{aligned}
& \left|\partial_{r} \underline{v}_{\underline{m}, \underline{p}, \underline{n}, \underline{q}}[w]\left(r, K^{(|\underline{m}|,|\underline{n}|)}\right)\right| \\
& \leq \quad\left(2\left\|\partial_{r} \chi_{1}\right\|_{\infty}\|F[w]\|_{\infty}^{L-1}+(L-1)\|F[w]\|_{\infty}^{L-2}\left\|\rho \partial_{r} F[w]\right\|_{\infty}\right) \\
& \quad \times \prod_{l=1}^{L} \frac{\left\|w_{m_{l}+p_{l}, n_{l}+q_{l}}\right\|_{\infty}}{\sqrt{p_{l} ! q_{l} !}} \\
& \quad+\|F[w]\|_{\infty}^{L-1} \sum_{l^{\prime}=1}^{L} \frac{\left\|\rho \partial_{r} w_{m_{l^{\prime}}+p_{l^{\prime}}, n_{l^{\prime}}+q_{l^{\prime}}}\right\|_{\infty}}{\sqrt{p_{l^{\prime}} ! q_{l^{\prime}} !}} \prod_{\substack{l=1 \\
l \neq l^{\prime}}}^{L} \frac{\left\|w_{m_{l}+p_{l}, n_{l}+q_{l}}\right\|_{\infty}}{\sqrt{p_{l} ! q_{l} !}} .
\end{aligned}
$$

Collecting estimates yields that claim.

Proof of Theorem 7.4. Assume $w \in \mathcal{B}^{\#}(\rho / 8, \rho / 2, \rho / 8)$. In view of the discussion in this section it remains to show that $\mathcal{R}_{\rho}^{\#}(w) \in \mathcal{W}_{\xi}^{\#}$. To this end note that by the definition of $\widehat{w}_{M, N},(7.5)$, we find for $M+N \geq 1$,

$$
\begin{aligned}
\left\|\widehat{w}_{M, N}\right\|^{\#} \leq & \sum_{L=1}^{\infty} \sum_{\substack{(\underline{m}, \underline{p}, \underline{n}, \underline{q}) \in \mathbb{N}_{0}^{4 L}: \\
|\underline{m}|=M,|\underline{n}|=N, m_{l}+p_{l}+n_{l}+q_{l} \geq 1}} \rho^{\underline{\underline{m}}|+| \underline{n} \mid-1} \\
& \times \prod_{l=1}^{L}\left\{\left(\begin{array}{c}
m_{l}+p_{l} \\
p_{l}
\end{array}\right)\left(\begin{array}{c}
n_{l}+q_{l} \\
q_{l}
\end{array}\right)\right\}\left\|v_{\underline{m}, \underline{p}, \underline{n}, \underline{q}}[w]\right\|^{\#} .
\end{aligned}
$$

Inserting this below and using the estimate of Lemma 7.5, we find using $\frac{1}{\sqrt{p_{l} ! q_{l} !}} \leq 1$

$$
\begin{aligned}
& \left\|\left(\widehat{w}_{M, N}\right)_{M+N \geq 1}\right\|_{\xi}^{\#} \\
& =\sum_{M+N \geq 1} \xi^{-(M+N)}\left\|\widehat{w}_{M, N}\right\|^{\#} \\
& \leq \sum_{L=1}^{\infty} C_{L} t^{1-L} \rho^{-1} \sum_{\substack{(\underline{m}, \underline{p}, \underline{n}, \underline{q}) \in \mathbb{N}_{0}^{4} L \\
|\underline{m}|+|\underline{n}| \geq 1, m_{l}+p_{l}+n_{l}+q_{l} \geq 1}}(2 \rho)^{|\underline{m}|+|\underline{n}|}(2 \xi)^{-(|\underline{m}|+|\underline{n}|)} \\
& \times \prod_{l=1}^{L}\left\{\left(\begin{array}{c}
m_{l}+p_{l} \\
p_{l}
\end{array}\right)\left(\begin{array}{c}
n_{l}+q_{l} \\
q_{l}
\end{array}\right) \frac{\left\|w_{m_{l}+p_{l}, n_{l}+q_{l}}\right\|^{\#}}{\sqrt{p_{l} ! q_{l} !}}\right\} \\
& \leq \sum_{L=1}^{\infty} C_{L} t^{-L} \sum_{\substack{(\underline{m}, \underline{p}, \underline{n}, \underline{q}) \in \mathbb{N}_{0}^{4 L}: \\
m_{l}+p_{l}+n_{l}+q_{l} \geq 1}} \\
& \times \prod_{l=1}^{L}\left\{\left(\begin{array}{c}
m_{l}+p_{l} \\
p_{l}
\end{array}\right)\left(\begin{array}{c}
n_{l}+q_{l} \\
q_{l}
\end{array}\right) \xi^{p_{l}+q_{l}}(1 / 2)^{m_{l}+n_{l}} \xi^{-\left(m_{l}+p_{l}+n_{l}+q_{l}\right)}\left\|w_{m_{l}+p_{l}, n_{l}+q_{l}}\right\|^{\#}\right\} \\
& \leq \sum_{L=1}^{\infty} C_{L} t^{-L}
\end{aligned}
$$




$$
\begin{aligned}
& \times\left[\sum_{m+p+n+q \geq 1}\left(\begin{array}{c}
m+p \\
p
\end{array}\right)\left(\begin{array}{c}
n+q \\
q
\end{array}\right) \xi^{p+q}(1 / 2)^{m+n} \xi^{-(m+p+n+q)}\left\|w_{m+p, n+q}\right\|^{\#}\right]^{L} \\
\leq & \sum_{L=1}^{\infty} C_{L} t^{-L}\left[\sum_{l+k \geq 1} \xi^{-(l+k)}\left\|w_{l, k}\right\|^{\#}\right]^{L} \\
\leq & \sum_{L=1}^{\infty} C_{L} t^{-L}\left(\left\|w_{\geq 1}\right\|_{\xi}^{\#}\right)^{L},
\end{aligned}
$$

where in the second last inequality we used the binomial formula

$$
\sum_{m+p=l}\left(\begin{array}{c}
m+p \\
p
\end{array}\right) \xi^{p}(1 / 2)^{m}=(\xi+1 / 2)^{l} \leq 1
$$

The term in the last line is bounded since $\left\|w_{\geq 1}\right\|_{\xi}^{\#} / t<1$. A similar but simpler estimate yields

$$
\begin{aligned}
\left\|\widehat{w}_{0,0}\right\|^{\#} & \leq \rho^{-1}\left\|w_{0,0}(\rho \cdot)\right\|^{\#}+\rho^{-1} \sum_{L=2}^{\infty} \sum_{\substack{\underline{p}, \underline{q}) \in \mathbb{N}_{0}^{2 L}: \\
p_{l}+q_{l} \geq 1}}\left\|v_{\underline{0}, \underline{p}, \underline{0}, \underline{q}}[w]\right\|^{\#} \\
& \leq \rho^{-1}\left\|w_{0,0}(\rho \cdot)\right\|^{\#}+\rho^{-1} \sum_{L=2}^{\infty} C_{L} t^{1-L} \sum_{(\underline{p}, \underline{q}) \in \mathbb{N}_{0}^{2 L}: l=1} \prod_{l=1}^{L} \frac{\left\|w_{p_{l}, q_{l}}\right\|^{\#}}{\sqrt{p_{l} ! q_{l} !}} \\
& \leq \rho^{-1}\left\|w_{0,0}(\rho \cdot)\right\|^{\#}+\sum_{L=2}^{\infty} C_{L}(\xi / t)^{L}\left[\sum_{p+q \geq 1} \xi^{-(p+q)}\left\|w_{p, q}\right\|^{\#}\right]^{L} \\
& \leq \rho^{-1}\left\|w_{0,0}(\rho \cdot)\right\|^{\#}+\sum_{L=2}^{\infty} C_{L}(\xi / t)^{L}\left(\left\|w_{\geq 1}\right\|_{\xi}^{\#}\right)^{L},
\end{aligned}
$$

where the last line is bounded since $\left\|w_{\geq 1}\right\|_{\xi}^{\#} / t<1$.

\section{Analyticity and Continuity}

In this section we show that the renormalization transformation acting on the integral kernels preserves analyticity and c-continuity, see Theorem 8.1, below. This result will imply on the one hand Theorem 8.2 , which states that the renormalized kernel is an analytic function of the spectral parameter, and on the other hand it will imply Theorem 8.3, which will be used to prove Theorem 10.4. Whenever we can treat a statement $A$ concerning analyticity and a statement $C$ concerning continuity in a similar way, we will write " $A(C)$ " which stands for " $A$ respectively $C$ ".

Theorem 8.1. Let $0<\rho \leq 1 / 2$ and $0<\xi \leq 1 / 2(0<\xi \leq 1 / 4)$. Let $S$ be an open subset of $\mathbb{C}^{\nu}$ with $\nu \in \mathbb{N}$ (a topological space). Suppose the map 
$w(\cdot): S \rightarrow \mathcal{W}_{\xi}^{\#}$ is analytic (c-continuous) and $w(S) \subset \mathcal{B}^{\#}(\rho / 8, \rho / 2, \rho / 8)$. Then

$$
\mathcal{R}_{\rho}^{\#} \circ w: S \rightarrow \mathcal{W}_{\xi}^{\#}
$$

is also analytic (c-continuous).

Lemma 6.1, Remark 6.4, Theorem 7.4, and Theorem 8.1 imply the following theorem:

Theorem 8.2. Let $0<\rho \leq 1 / 2$ and $0<\xi \leq 1 / 2$. For $w \in \mathcal{B}(\rho / 8, \rho / 8, \rho / 8)$ the function defined by $\mathcal{R}_{\rho} w:=\mathcal{R}_{\rho}^{\#} \circ w \circ E_{\rho}[w]^{-1}: D_{1 / 2} \rightarrow \mathcal{W}_{\xi}^{\#}$ is in $\mathcal{W}_{\xi}$. I.e., for $z \in D_{1 / 2}$ we have $\left(\mathcal{R}_{\rho} w\right)(z)=\mathcal{R}_{\rho}^{\#}\left(w\left(E_{\rho}[w]^{-1}(z)\right)\right.$. Moreover,

$$
R_{\rho}(H(w))=H\left(\mathcal{R}_{\rho} w\right) .
$$

If $w$ is symmetric, then also $\mathcal{R}_{\rho} w$ is symmetric.

The statement about the symmetry follows from (5.14) and the definition of the renormalization transformation, see Definition 6.5. The symmetry property could also be verified using the explicit expressions (7.5) and (7.6). We write F-differentiable for Frechet differentiable. Furthermore, Theorem 8.1 has the following theorem as consequence:

Theorem 8.3. Let $0<\rho \leq 1 / 2$ and $0<\xi \leq 1 / 2(0<\xi \leq 1 / 4)$. Let $S$ be an open subset of $\mathbb{C}^{\nu}$ (a topological space). Suppose

$$
\begin{aligned}
w(\cdot, \cdot): & S \times D_{1 / 2} \rightarrow \mathcal{W}_{\xi}^{\#} \\
& (s, z) \mapsto w(s, z)
\end{aligned}
$$

is an analytic (a c-continuous) function such that $w(s)(\cdot):=w(s, \cdot)$ is in $\mathcal{B}(\rho / 8, \rho / 8, \rho / 8)$. Then,

$$
(s, z) \mapsto\left(\mathcal{R}_{\rho}(w(s))\right)(z)
$$

is also a $\mathcal{W}_{\xi}^{\#}$-valued analytic (c-continuous) function.

Proof. First observe that $(s, z) \mapsto E_{\rho}[w(s)](z)$ is analytic (continuous). It follows that the mapping $(s, z) \mapsto E_{\rho}[w(s)]^{-1}(z)$ on $S \times D_{1 / 2}$ is analytic (continuous), which can be seen from Lemma 6.1 and the identity

$$
E_{\rho}[w(s)]\left(E_{\rho}[w(s)]^{-1}(z)\right)-z=0 .
$$

It follows that the map $(s, z) \in S \times D_{1 / 2} \rightarrow w\left(s, E_{\rho}[w(s)]^{-1}(z)\right)$ is analytic (c-continuous) and by Remark 6.4 its range is contained in $\mathcal{B}^{\#}(\rho / 8, \rho / 2, \rho / 8)$. It follows now from Theorem 8.1 that $(s, z) \mapsto \mathcal{R}_{\rho}^{\#}\left(w\left(s, E_{\rho}[w(s)]^{-1}(z)\right)\right)$ is analytic (c-continuous).

Remark 8.4. Note that by Hartogs' Theorem joint analyticity is equivalent to individual analyticity.

The remaining part of this section is devoted to the proof of Theorem 8.1. We note that an alternative proof of that theorem would be to show that $\mathcal{R}_{\rho}^{\#}$ is a Frechet differentiable map on the space of integral kernels. We will 
follow a different path and show that the renormalized kernel is given as a convergent sum of analytic (c-continuous) terms.

First we show the statement regarding analyticity, then we show the statement regarding c-continuity. To show the statement about analyticity we first show in Lemma 8.5, below, that the map $v_{\underline{m}, \underline{p}, \underline{n}, \underline{q}}[w(\cdot)]: S \rightarrow \mathcal{W}_{\xi}^{\#}$ is analytic. It then follows from (7.5) and (7.6) that the renormalized kernel $\widehat{w(s)}=\mathcal{R}_{\rho}^{\#}(w(s))$ is given as a series of analytic mappings. Analyticity of the renormalized kernel will follow, provided that the series converges uniformly on $S$. Since we are not able to show this on the whole set $S$ directly, we will show, below, uniform convergence on open subsets of $S$ which constitute a covering of $S$. This is in fact sufficient to conclude the analyticity of $s \mapsto \mathcal{R}_{\rho}^{\#}(w(s))$.

Lemma 8.5. Let the assumptions of Theorem 8.1 hold. Then, $v_{\underline{m}, \underline{p}, \underline{n}, \underline{q}}[w(\cdot)]$ : $S \rightarrow \mathcal{W}_{\xi}^{\#}$ is analytic.

Lemma 8.5 follows since by part (a) of the following Lemma and Estimate $(6.4)$ the function $v_{\underline{m}, \underline{p}, \underline{n}, \underline{q}}[w(\cdot)]$ is a composition of an analytic map with a F-differentiable map.

Lemma 8.6. Let $0<\rho \leq 1 / 2$. Then the following statements hold for $\epsilon>0$ :

(a) On $\mathcal{O}^{(\epsilon)}:=\left\{w \in \mathcal{W}_{\xi}^{\#}\left|\inf _{r \in\left[\rho \frac{3}{4}, 1\right]}\right| w_{0,0}(r) \mid>\epsilon\right\}$ the following map is F-differentiable

$$
v_{\underline{m}, \underline{p}, \underline{n}, \underline{q}}[\cdot]: \mathcal{O}^{(\epsilon)} \longrightarrow \mathcal{W}_{\xi}^{\#}, \quad w \longmapsto v_{\underline{m}, \underline{p}, \underline{n}, \underline{q}}[w] .
$$

(b) On $\mathcal{O}_{0,0}^{(\epsilon)}:=\left\{t \in \mathcal{W}_{0,0}^{\#}\left|\inf _{r \in\left[\rho \frac{3}{4}, 1\right]}\right| t(r) \mid>\epsilon\right\}$ the following map is F-differentiable

$$
F[\cdot]: \mathcal{O}_{0,0}^{(\epsilon)} \longrightarrow \mathcal{W}_{0,0}^{\#}, \quad t \longmapsto \frac{\bar{\chi}_{\rho}^{2}}{t} .
$$

Proof. First we show part (b). We will use that for all $f, g \in \mathcal{W}_{0,0}$ we have $\|f g\|^{\#} \leq\|f\|^{\#}\|g\|^{\#}$ and that for all $\xi \in \mathcal{W}_{0,0}$ with $\|\xi\|^{\#}<\epsilon / 2$ we have

$$
\begin{aligned}
\left\|F[t+\xi]-F[t]+\frac{\bar{\chi}_{\rho}^{2} \xi}{t^{2}}\right\|^{\#} & =\left\|\frac{\bar{\chi}_{\rho}^{2} \xi^{2}}{t^{2}(t+\xi)}\right\|^{\#} \\
& \leq\left\|\frac{\bar{\chi}_{\rho}}{t^{2}}\right\|^{\#}\left\|\frac{\bar{\chi}_{\rho}}{t+\xi}\right\|^{\#}\left\|\xi^{2}\right\|^{\#} \leq C\left(\|\xi\|^{\#}\right)^{2},
\end{aligned}
$$

where in the last inequality we used the estimate

$$
\begin{aligned}
\left\|\frac{\bar{\chi}_{\rho}}{t+\xi}\right\|^{\#} & \leq\left\|\frac{\bar{\chi}_{\rho}}{t+\xi}\right\|_{\infty}+\left\|\frac{\partial_{r} \bar{\chi}_{\rho}}{t+\xi}\right\|_{\infty}+\left\|\frac{\bar{\chi}_{\rho} \partial_{r}(t+\xi)}{(t+\xi)^{2}}\right\|_{\infty} \\
& \leq \frac{1+\left\|\partial_{r} \bar{\chi}_{\rho}\right\|_{\infty}}{\epsilon-\|\xi\|^{\#}}+\frac{\|t\|^{\#}+\|\xi\|^{\#}}{\left(\epsilon-\|\xi\|^{\#}\right)^{2}} \leq C .
\end{aligned}
$$

This implies that $F[\cdot]$ is differentiable with derivative $-\bar{\chi}_{\rho}^{2} / t^{2}$.

(b) The differentiability of $v_{\underline{m}, \underline{p}, \underline{n}, \underline{q}}[\cdot]$ follows from the fact it can be written as a composition of the F-differentiable mapping $\widetilde{v}_{\underline{m}, \underline{p}, \underline{n}, \underline{q}}[\cdot]$, defined below, 
and $F[\cdot]$. For $\underline{w}=\left(w_{1}, \ldots, w_{L}\right) \in\left(\mathcal{W}_{\xi}^{\#}\right)^{L}$ and $\underline{G}=\left(G_{0}, \ldots, G_{L}\right) \in\left(\mathcal{W}_{0,0}^{\#}\right)^{L+1}$ define the multilinear expression

$$
\begin{aligned}
& \widetilde{\widetilde{v}}_{\underline{m}, \underline{p}, \underline{n}, \underline{q}}[\underline{w}, \underline{G}]\left(r, K^{(|\underline{m}|,|\underline{n}|)}\right):=\left\langle\Omega, G_{0}\left(H_{f}+\rho\left(r+\widetilde{r}_{0}\right)\right)\right. \\
& \left.\quad \times \prod_{l=1}^{L}\left\{W_{p_{l}, q_{l}}^{m_{l}, n_{l}}\left[w_{l}\right]\left(\rho\left(r+r_{l}\right), \rho K_{l}^{\left(m_{l}, n_{l}\right)}\right) G_{l}\left(H_{f}+\rho\left(r+\widetilde{r}_{l}\right)\right)\right\} \Omega\right\rangle .
\end{aligned}
$$

It satisfies the inequality

$$
\begin{aligned}
& \left\|\widetilde{v}_{\underline{m}, \underline{p}, \underline{n}, \underline{q}}[\underline{w}, \underline{G}]\right\|^{\#} \\
& \quad \leq\left(\prod_{l=0}^{L}\left\|G_{l}\right\|_{\infty}+\sum_{l^{\prime}=0}^{L}\left\|\rho \partial_{r} G_{l^{\prime}}\right\|_{\infty} \prod_{l=0, l \neq l^{\prime}}^{L}\left\|G_{l}\right\|_{\infty}\right) \prod_{l=1}^{L} \frac{\left\|\left(w_{l}\right)_{m_{l}+p_{l}, n_{l}+q_{l}}\right\|^{\#}}{\sqrt{p_{l} ! q_{l} !}} .
\end{aligned}
$$

To obtain (8.6) we use (7.3) and Lemma 7.1, and calculate the derivative with respect to $r$ using Leibniz' rule. From (8.6) it follows that $\widetilde{v}_{\underline{m}, \underline{p}, \underline{n}, q}[\cdot]$ is continuous, and hence by multilinearity $\widetilde{v}_{\underline{m}, \underline{p}, \underline{n}, \underline{q}}[\cdot]$ is in fact differentiable.

Next we show that the defining sequence of $\widehat{w(s)}$, see $(7.5)$ and $(7.6)$, converges uniformly on open sets which constitute a covering of $S$. To this end choose $s_{0} \in S$ and define the set

$$
U_{0}=\left\{w \in \mathcal{B}^{\#}(\rho / 8, \rho / 2, \rho / 8) \mid\left\|w-w\left(s_{0}\right)\right\|_{\xi}^{\#}<\epsilon\right\}
$$

where we set

$$
\epsilon:=\frac{\rho / 7-\left\|w\left(s_{0}\right)_{\geq 1}\right\|_{\xi}^{\#}}{16 \mathrm{e}^{4}} .
$$

The explicit choice of $\epsilon$ is needed for the estimate (8.9), below. Note that by continuity there exists, $S_{0}$, an open subset of $S$ containing $s_{0}$, such that $w\left(S_{0}\right) \subset U_{0}$. For $w \in U_{0}$, we have

$$
\left\|w_{m, n}\right\|^{\#} \leq E_{m, n}:=\left\|w\left(s_{0}\right)_{m, n}\right\|^{\#}+\xi^{m+n} \epsilon .
$$

By Lemma 7.5,

$$
\sup _{s \in S_{0}}\left\|v_{\underline{m}, \underline{p}, \underline{n}, \underline{q}}[w(s)]\right\|^{\#} \leq C_{L} t^{-L+1} \prod_{l=1}^{L} \frac{E_{m_{l}+p_{l}, n_{l} q_{l}}}{\sqrt{p_{l} ! q_{l} !}}
$$

where we used the notation introduced in that lemma. To establish the uniform convergence on $S_{0}$ of the series defining $\widehat{w(s)}$ it suffices, in view of (7.5) and (7.6), to show that the following expression is bounded: 


$$
\begin{aligned}
& \sum_{M+N \geq 0} \sum_{L=1}^{\infty} \sum_{\substack{(\underline{m}, \underline{p}, \underline{n}, \underline{q}) \in \mathbb{N}_{0}^{4 L} \\
|\underline{m}|=M,|\underline{n}|=N \\
m_{l}+p_{l}+n_{l}+q_{l} \geq 1}} \xi^{-|\underline{m}|-|\underline{n}|} \rho^{|\underline{m}|+|\underline{n}|} \prod_{l=1}^{L}\left\{\left(\begin{array}{c}
m_{l}+p_{l} \\
p_{l}
\end{array}\right)\left(\begin{array}{c}
n_{l}+q_{l} \\
q_{l}
\end{array}\right)\right\} \\
& \times \sup _{s \in S_{0}}\left\|v_{\underline{m}, \underline{p}, \underline{n}, \underline{q}}[w(s)]\right\|^{\#} \leq \sum_{L=1}^{\infty} C_{L} t^{1-L} G^{L}
\end{aligned}
$$

where we used Eq. (8.7) and the definition

$$
G:=\sum_{m+p+n+q \geq 1}\left(\begin{array}{c}
m+p \\
p
\end{array}\right)\left(\begin{array}{c}
n+q \\
q
\end{array}\right) \xi^{p+q}(1 / 2)^{m+n} \xi^{-m-p-n-q} \frac{E_{m+p, n+q}}{\sqrt{p ! q !}} .
$$

Below we will show that

$$
G \leq\left\|w\left(s_{0}\right)_{\geq 1}\right\|_{\xi}^{\#}+\epsilon 16 \mathrm{e}^{4} \leq \rho / 7
$$

Inequalities (8.9) imply the convergence of (8.8), since $t^{-1} G \leq t^{-1} \rho / 7<1$. The second inequality in (8.9) follows from the definition of $\epsilon$. To show the first inequality of (8.9), we will use the following estimate:

$$
\begin{aligned}
& \sum_{m+p \geq 0}\left(\begin{array}{c}
m+p \\
p
\end{array}\right) \xi^{p}(1 / 2)^{m} \frac{1}{\sqrt{p !}} \\
& \leq \sum_{m+p \geq 0}\left(\begin{array}{c}
m+p \\
p
\end{array}\right)(1 / 4)^{p}(1 / 2)^{m} \mathrm{e}^{8 \xi^{2}}=4 \mathrm{e}^{8 \xi^{2}} \leq 4 \mathrm{e}^{2},
\end{aligned}
$$

where in the first inequality we used the trivial estimate $\left(16 \xi^{2}\right)^{p} / p ! \leq \mathrm{e}^{16 \xi^{2}}$. To show the first inequality in (8.9), we insert the definition of $E_{m, n}$ into the definition of $G$. This yields two terms, which we have to estimate. The first term, involving $w_{m, n}\left(s_{0}\right)$, is estimated using the binomial formula and the second term, involving $\epsilon$, is estimated using (8.10).

It remains to show the statement regarding c-continuity. By Lemma 8.7, shown next, the map $s \mapsto v_{\underline{m}, \underline{p}, \underline{n}, \underline{q}}[w(s)]$ is continuous with respect to $\|\cdot\|_{2}$. By (7.5) and (7.6) this will imply that the function $s \mapsto \widehat{w(s)}{ }_{M, N}$ is given as a series involving expressions which are continuous with respect to $\|\cdot\|_{2}$. The ccontinuity of $s \mapsto \widehat{w(s)}$ will follow provided we show that this series converges uniformly in $s \in S$ with respect to the $\|\cdot\|_{2}$ norm. In fact we will first show uniform convergence with respect to $\|\cdot\|^{\#}$. In view of the second inequality of (5.9) this will imply the uniform convergence with respect to the $\|\cdot\|_{2}$ norm.

Lemma 8.7. Let $w: S \mapsto \mathcal{W}_{\xi}^{\#}$ be c-continuous, and let $w(S) \subset \mathcal{B}^{\#}$ $(\rho / 8, \rho / 2, \rho / 8)$. Then, for all $s_{0} \in S$

$$
\lim _{s \in S, s \rightarrow s_{0}}\left\|v_{\underline{m}, \underline{p}, \underline{n}, \underline{q}}\left[w\left(s_{0}\right)\right]-v_{\underline{m}, \underline{p}, \underline{n}, \underline{q}}[w(s)]\right\|_{2} \rightarrow 0 .
$$


Proof. The kernel $v_{\underline{m}, \underline{p}, \underline{n}, \underline{q}}$ is a multilinear expression of integral kernels. To show continuity we use the following identity:

$$
\begin{aligned}
& A_{1}(s) \cdots A_{n}(s)-A_{1}\left(s_{0}\right) \cdots A_{n}\left(s_{0}\right) \\
& \quad=\sum_{i=1}^{n} A_{1}(s) \cdots A_{i-1}(s)\left(A_{i}(s)-A_{i}\left(s_{0}\right)\right) A_{i+1}\left(s_{0}\right) \cdots A_{n}\left(s_{0}\right) .
\end{aligned}
$$

Now (8.11) follows using estimate (7.3), the following inequality, which is shown similarly as the estimate in Lemma 5.3,

$$
\int \frac{\mathrm{d} K^{(m, n)}}{\left|K^{(m, n)}\right|^{2}} \sup _{r \in[0,1]}\left\|W_{p, q}^{m, n}[w]\left(r, K^{m, n}\right)\right\|_{\mathrm{op}}^{2} \leq\left\|w_{m+p, n+q}\right\|_{2}^{2},
$$

the second inequality of (5.9), and the limits

$$
\begin{array}{r}
\left\|w\left(s_{0}\right)_{m, n}-w(s)_{m, n}\right\|_{2} \stackrel{s \rightarrow s_{0}}{\longrightarrow} 0, \\
\sup _{r}\left|\frac{\bar{\chi}_{1}^{2}(r)}{w\left(s_{0}\right)_{0,0}(r)}-\frac{\bar{\chi}_{1}^{2}(r)}{w(s)_{0,0}(r)}\right| \stackrel{s \rightarrow s_{0}}{\longrightarrow} 0,
\end{array}
$$

which follow by assumption.

It remains to show that the defining series of $\widehat{w(s)}{ }_{M, N}$ converges uniformly in $s \in S$. In view of (7.5) and (7.6) this will be established if we can show that (8.15) and (8.18) are finite. To this end, first observe that it follows that for all $m+n \geq 1$

$$
\sup _{s \in S}\left\|w(s)_{m, n}\right\|^{\#} \leq \xi^{m+n} \sup _{s \in S}\left\|\left(w(s)_{m, n}\right)_{m+n \geq 1}\right\|_{\xi}^{\#} \leq \xi^{m+n} \frac{\rho}{8} .
$$

Inserting (7.8) and the above estimate into the following expression, for $M+$ $N \geq 1$, we find

$$
\begin{aligned}
& \sum_{L=1}^{\infty} \sum_{\substack{(\underline{m}, \underline{n}) \in \mathbb{N}_{0}^{2 L}: \\
|\underline{m}|=M,|\underline{n}|=N}} \sum_{\substack{(\underline{p}, \underline{q}) \in \mathbb{N}_{0}^{2 L} \\
m_{l}+\underline{p}+n_{l}+n_{l} \geq 1}} \prod_{l=1}^{L}\left\{\left(\begin{array}{c}
m_{l}+p_{l} \\
p_{l}
\end{array}\right)\left(\begin{array}{c}
n_{l}+q_{l} \\
q_{l}
\end{array}\right)\right\} \\
& \times \sup _{s \in S}\left\|v_{\underline{m}, \underline{p}, \underline{n}, \underline{q}}[w(s)]\right\| \# \\
& \leq \sum_{L=1}^{\infty}(-1)^{L-1} \rho^{M+N-1} C_{L} t^{1-L} \sum_{\substack{(\underline{m}, \underline{n}) \in \mathbb{N}_{0}^{2 L}: \\
|\underline{m}|=M,|\underline{n}|=N}} \\
& \times \prod_{l=1}^{L}\left\{\sum_{p_{l}, q_{l}}^{\prime}\left(\begin{array}{c}
m_{l}+p_{l} \\
p_{l}
\end{array}\right)\left(\begin{array}{c}
n_{l}+q_{l} \\
q_{l}
\end{array}\right) \xi^{m_{l}+p_{l}+n_{l}+q_{l}} \frac{\rho}{8}\right\} \\
& =: F \text {, }
\end{aligned}
$$


where $\sum_{p_{l}, q_{l}}^{\prime}$ denotes the sum over all $\left(p_{l}, q_{l}\right) \in \mathbb{N}_{0}^{2}$ such that $m_{l}+p_{l}+n_{l}+q_{l} \geq 1$. If $m_{l} \neq 0$ or $n_{l} \neq 0$ we estimate using $\left(\begin{array}{l}n \\ k\end{array}\right) \leq 2^{n}$

$$
\begin{aligned}
\sum_{p_{l}, q_{l}}^{\prime}\left(\begin{array}{c}
m_{l}+p_{l} \\
p_{l}
\end{array}\right)\left(\begin{array}{c}
n_{l}+q_{l} \\
q_{l}
\end{array}\right) \xi^{m_{l}+p_{l}+n_{l}+q_{l}} & \leq(2 \xi)^{m_{l}+n_{l}} \sum_{p_{l} \geq 0}(2 \xi)^{p_{l}} \sum_{q_{l} \geq 0}(2 \xi)^{q_{l}} \\
& \leq 4,
\end{aligned}
$$

where we used that $0<\xi \leq 1 / 4$. If both $m_{l}=0$ and $n_{l}=0$ then either $p_{l} \geq 1$ or $q_{l} \geq 1$ and we estimate

$$
\sum_{p_{l}, q_{l}}^{\prime}\left(\begin{array}{c}
m_{l}+p_{l} \\
p_{l}
\end{array}\right)\left(\begin{array}{c}
n_{l}+q_{l} \\
q_{l}
\end{array}\right) \xi^{m_{l}+p_{l}+n_{l}+q_{l}} \leq \sum_{p_{l}+q_{l} \geq 1} \xi^{p_{l}} \xi^{q_{l}} \leq \frac{7}{9} \leq 1,
$$

where in the second last inequality we used again $0<\xi \leq 1 / 4$. Inserting these estimates into $F$ and using that there are at most $(M+1)(N+1)$ factors for which $m_{l} \neq 0$ or $n_{l} \neq 0$ we find

$$
F \leq \rho^{M+N-1} \sum_{L=1}^{\infty} C_{L} t^{1-L} \sum_{\substack{(\underline{m}, \underline{n}) \in \mathbb{N}_{0}^{2 L}: \\|\underline{m}|=M,|\underline{n}|=N}} 4^{(M+1)(N+1)}\left(\frac{\rho}{8}\right)^{L}
$$

Now the estimates

$$
\sum_{\substack{(\underline{m}, \underline{n}) \in \mathbb{N}_{0}^{2 L}: \\|\underline{m}|=M,|\underline{n}|=N}} 1 \leq(L+1)^{M+N}
$$

and $t^{-1} \rho / 8<1$ imply $F<\infty$. Now we consider (7.6). Using (7.8) and (8.14) we find

$$
\sum_{L=2}^{\infty} \sum_{\substack{\underline{p}, \underline{q}) \in \mathbb{N}_{0}^{2 L}: \\ p_{l}+q_{l} \geq 1}}\left\{\sup _{s \in S}\left\|v_{\underline{0}, \underline{p}, \underline{0}, \underline{q}}[w(s)]\right\|^{\#}\right\} \leq \sum_{L=2}^{\infty} C_{L} t^{L-1}\left\{\sum_{p+q \geq 1} \xi^{p+q} \frac{\rho}{8}\right\}^{L} .
$$

This converges since $\sum_{p+q \geq 1} \xi^{p+q} \leq 7 / 9 \leq 1$ and $t^{-1} \rho / 8<1$.

\section{Codimension-1 Contractivity}

In this section we prove that the renormalization transformation is in certain directions a contraction in $\mathcal{W}_{\xi}$. We recall Definition 6.5 and the definition introduced in Theorem 8.2. In contrast to [2], the contraction originates from the fact the we restrict the renormalization transformation to integral kernels for which the sum of the number of creation and annihilation operators is even, rather than an infrared condition.

Theorem 9.1. For any positive numbers $\rho_{0} \leq 1 / 2$ and $\xi_{0} \leq 1 / 2$ there exist numbers $\rho, \xi, \epsilon_{0}$ satisfying $\rho \in\left(0, \rho_{0}\right], \xi \in\left(0, \xi_{0}\right]$, and $0<\epsilon_{0} \leq \rho / 8$ such that the following property holds:

$$
\mathcal{R}_{\rho}: \mathcal{B}_{0}\left(\epsilon, \delta_{1}, \delta_{2}\right) \rightarrow \mathcal{B}_{0}\left(\epsilon+\delta_{2} / 2, \delta_{2} / 2, \delta_{2} / 2\right), \quad \forall \epsilon, \delta_{1}, \delta_{2} \in\left[0, \epsilon_{0}\right) .
$$


In fact we will prove the following remark which is a slightly stronger statement than Theorem 9.1:

Remark 9.2. Define the constant $C_{\theta}:=3+2\left\|\partial_{r} \chi_{1}\right\|_{\infty}$. The contraction property (9.1) holds whenever $0<\rho \leq \frac{1}{16 C_{\theta}}, 0<\xi \leq\left[\rho /\left(2 C_{\theta}\right)\right]^{1 / 4}$, and $0<\epsilon_{0} \leq \frac{\rho}{32}$.

Proof. We will prove Remark 9.2. Theorem 9.1 will then follow. First observe that if $w \in \mathcal{B}_{0}\left(\epsilon, \delta_{1}, \delta_{2}\right)$, then $\left(\mathcal{R}_{\rho} w\right)_{m, n}=0$, if $m+n$ is odd. Since $C_{\theta} \geq 1$, we can assume that $\xi \leq 1 / 2$ and $\rho \leq 1 / 2$. To show the contraction property, we will use the following estimate for $w \in \mathcal{B}^{\#}(\rho / 8, \rho / 2, \rho / 8)$ :

$$
\left\|v_{\underline{m}, \underline{p}, \underline{n}, \underline{q}}[w]\right\|^{\#} \leq C_{\theta}\left(\frac{16}{\rho}\right)^{L-1} \prod_{l=1}^{L} \frac{\left\|w_{m_{l}+p_{l}, n_{l}+q_{l}}\right\|^{\#}}{\sqrt{p_{l} ! q_{l} !}},
$$

which follows directly from Lemma 7.5. We shall use the notation $z=E_{\rho}[w]^{-1}$ $(\zeta)$ where $\zeta \in D_{1 / 2}$, see Lemma 6.1 .

Step 1: We have

$$
\left\|\left(\mathcal{R}_{\rho} w\right)_{\geq 2}\right\|_{\xi} \leq \frac{1}{2}\left\|w_{\geq 2}\right\|_{\xi}
$$

By the definition of $\widehat{w}_{M, N},(7.5)$, we find for $M+N \geq 2$,

$$
\begin{aligned}
\left\|\widehat{w(z)}_{M, N}\right\|^{\#} \leq & \sum_{L=1}^{\infty} \sum_{\substack{(\underline{m}, \underline{p}, \underline{n}, \underline{q}) \in \mathbb{N}_{0}^{4} L \\
|\underline{m}|=M,|\underline{n}|=N, m_{l}+p_{l}+n_{l}+q_{l} \geq 1}} \rho^{|\underline{m}|+|\underline{n}|-1} \\
& \times \prod_{l=1}^{L}\left(\begin{array}{c}
m_{l}+p_{l} \\
p_{l}
\end{array}\right)\left(\begin{array}{c}
n_{l}+q_{l} \\
q_{l}
\end{array}\right)\left\|v_{\underline{m}, \underline{p}, \underline{n}, \underline{q}}[w(z)]\right\|^{\#}
\end{aligned}
$$

Inserting this below and using the Estimate (9.2) we find with $\tau:=16 / \rho$,

$$
\begin{aligned}
& \left\|\left(\left(\mathcal{R}_{\rho} w\right)(\zeta)\right)_{M+N \geq 2}\right\|_{\xi}^{\#} \\
& =\sum_{M+N \geq 2} \xi^{-(M+N)}\left\|\widehat{w(z)}_{M, N}\right\|^{\#} \\
& \leq \sum_{L=1}^{\infty} \sum_{\substack{(\underline{m}, \underline{p}, \underline{n}, \underline{q}) \in \mathbb{N}_{0}^{4} L: \\
|\underline{m}|+|\underline{n}| \geq 2, m_{l}+p_{l}+n_{l}+q_{l} \geq 1}} \rho^{-1}(2 \rho)^{|\underline{m}|+|\underline{n}|}(2 \xi)^{-(|\underline{m}|+|\underline{n}|)} C_{\theta} \tau^{L-1} \\
& \times \prod_{l=1}^{L}\left\{\left(\begin{array}{c}
m_{l}+p_{l} \\
p_{l}
\end{array}\right)\left(\begin{array}{c}
n_{l}+q_{l} \\
q_{l}
\end{array}\right) \frac{\left\|w(z)_{m_{l}+p_{l}, n_{l}+q_{l}}\right\|^{\#}}{\sqrt{p_{l} ! q_{l} !}}\right\} \\
& \leq \frac{C_{\theta}}{16}[2 \rho]^{2} \sum_{L=1}^{\infty} \tau^{L} \sum_{\substack{(\underline{m}, \underline{p}, \underline{\underline{n}}, \underline{q}) \in \mathbb{N}_{0}^{4} \\
m_{l}+\underline{p}_{l}+n_{l}+q_{l} \geq 1}} \prod_{l=1}^{L} \\
& \times\left\{\left(\begin{array}{c}
m_{l}+p_{l} \\
p_{l}
\end{array}\right)\left(\begin{array}{c}
n_{l}+q_{l} \\
q_{l}
\end{array}\right) \xi^{p_{l}+q_{l}} 2^{-\left(m_{l}+n_{l}\right)} \xi^{-\left(m_{l}+p_{l}+n_{l}+q_{l}\right)}\left\|w(z)_{m_{l}+p_{l}, n_{l}+q_{l}}\right\|^{\#}\right\} \\
& \leq \frac{C_{\theta}}{4} \rho^{2} \sum_{L=1}^{\infty} \tau^{L}\left[\sum_{m+p+n+q \geq 1}\left(\begin{array}{c}
m+p \\
p
\end{array}\right)\left(\begin{array}{c}
n+q \\
q
\end{array}\right)\right.
\end{aligned}
$$




$$
\begin{aligned}
& \left.\times \xi^{p+q} 2^{-(m+n)} \xi^{-(m+p+n+q)}\left\|w(z)_{m+p, n+q}\right\|^{\#}\right]^{L} \\
\leq & \frac{C_{\theta}}{4} \rho^{2} \sum_{L=1}^{\infty} \tau^{L}\left[\sum_{l+k \geq 1} \xi^{-(l+k)}\left\|w(z)_{l, k}\right\|^{\#}\right]^{L} \\
\leq & \frac{C_{\theta}}{4} \rho^{2} \sum_{L=1}^{\infty} \tau^{L}\left(\left\|w(z)_{\geq 2}\right\|_{\xi}^{\#}\right)^{L} \\
\leq & 8 C_{\theta} \rho\left\|w(z)_{\geq 2}\right\|_{\xi}^{\#},
\end{aligned}
$$

where in the third last inequality we used the binomial formula, (7.15), and we used $\tau\left\|w_{\geq 2}\right\|_{\xi} \leq 1 / 2$ in the last inequality.

Step 2:

$\sup _{\zeta \in D_{1 / 2}}\left\|\partial_{r}\left(\mathcal{R}_{\rho} w\right)(\zeta)_{0,0}-1\right\|_{\infty} \leq \sup _{z \in D_{1 / 2}}\left\|\partial_{r} w(z)_{0,0}-1\right\|_{\infty}+\frac{1}{2}\left\|w_{\geq 1}\right\|_{\xi}$

Using the definition of $\widehat{w}_{0,0},(7.6)$, we find,

$$
\begin{aligned}
& \left\|\partial_{r}\left(\mathcal{R}_{\rho} w\right)(\zeta)_{0,0}-1\right\|_{\infty} \\
& \leq\left\|\partial_{r} w(z)_{0,0}-1\right\|_{\infty}+\rho^{-1} \sum_{L=2}^{\infty} \sum_{\substack{\underline{p}, \underline{q}) \in \mathbb{N}_{0}^{2 L}: \\
p_{l}+q_{l} \geq 1}}\left\|v_{\underline{0}, \underline{p}, \underline{\underline{0}}, \underline{\underline{x}}}[w(z)]\right\|^{\#} \\
& \quad \leq\left\|\partial_{r} w(z)_{0,0}-1\right\|_{\infty}+\rho^{-1} \sum_{L=2}^{\infty} C_{\theta} \tau^{L-1} \sum_{(\underline{p}, \underline{q}) \in \mathbb{N}_{0}^{2 L}: l=1} \prod_{l}^{L} \frac{\left\|w(z)_{p_{l}, q_{l}}\right\|^{\#}}{\sqrt{p_{l} ! q_{l} !}} \\
& \leq\left\|\partial_{r} w(z)_{0,0}-1\right\|_{\infty}+\frac{C_{\theta}}{16} \sum_{L=2}^{\infty}\left[\tau \xi^{2}\right]^{L}\left[\sum_{p+q \geq 2} \xi^{-(p+q)}\left\|w(z)_{p, q}\right\|^{\#}\right]^{L} \\
& \leq\left\|\partial_{r} w(z)_{0,0}-1\right\|_{\infty}+\frac{C_{\theta}}{16} \xi^{4} \sum_{L=2}^{\infty}\left[\tau\left\|w(z)_{\geq 2}\right\|_{\xi}^{\#}\right]^{L} \\
& \leq\left\|\partial_{r} w(z)_{0,0}-1\right\|_{\infty}+\frac{C_{\theta}}{16} \xi^{4} \tau\left\|w(z)_{\geq 2}\right\|_{\xi}^{\#}
\end{aligned}
$$

where in the last estimate we used $\tau\left\|w_{\geq 1}\right\|_{\xi} \leq 1 / 2$.

Step 3:

$$
\sup _{\zeta \in D_{1 / 2}}\left|\left(\mathcal{R}_{\rho} w\right)(\zeta)_{0,0}(0)+\zeta\right| \leq \frac{1}{2}\left\|w_{\geq 1}\right\|_{\xi}
$$


We estimate

$$
\begin{aligned}
\left|\left(\mathcal{R}_{\rho} w\right)(\zeta)_{0,0}(0)+\zeta\right| & \leq \rho^{-1} \sum_{L=2}^{\infty} \sum_{\substack{\underline{p}, \underline{q}) \in \mathbb{N}_{0}^{2 L} \\
\underline{p}_{l}+q_{l} \geq 1}}\left\|v_{\underline{0}, \underline{p}, \underline{0}, \underline{q}}[w(z)]\right\|^{\#} \\
& \leq \frac{C_{\theta}}{16} \xi^{4} \tau\left\|w(z)_{\geq 1}\right\|_{\xi}^{\#},
\end{aligned}
$$

where in the last step we used an estimate from Step 2.

\section{Construction of Eigenvectors and Eigenvalues}

In this section we show how the contraction property of Theorem 9.1 and the Feshbach property allows us to recover the eigenvectors and eigenvalues of the initial operator. The main theorems of this section are Theorems 10.3 and 10.4. Theorem 10.3, apart from the last sentence, is from [2]. We follow the proof given there, and isolate a few estimates which will be needed to prove the analyticity and continuity results of Theorem 2.4. The last sentence in Theorem 10.3, has been shown in [14] but in a different way, due to the different representation of the spectral parameter in [14].

Throughout this section we assume the following hypothesis:

(R) Let $\rho, \xi, \epsilon_{0}$ be positive numbers such that the contraction property (9.1) holds and $\rho \leq 1 / 4, \xi \leq 1 / 4$ and $\epsilon_{0} \leq \rho / 8$.

We note that many statements only require $0<\rho \leq 1 / 2$ and $0<\xi \leq 1 / 2$. But we will need $0<\rho \leq 1 / 4$ in Lemma 10.1, below, and we will need $0<\xi \leq 1 / 4$ for the statement about c-continuity in Theorem 8.3. Hypothesis (R) allows us to iterate the renormalization transformation as follows:

$$
\begin{array}{r}
\mathcal{B}_{0}\left(\frac{1}{2} \epsilon_{0}, \frac{1}{2} \epsilon_{0}, \frac{1}{2} \epsilon_{0}\right) \stackrel{\mathcal{R}_{\rho}}{\longrightarrow} \mathcal{B}_{0}\left(\left[\frac{1}{2}+\frac{1}{4}\right] \epsilon_{0}, \frac{1}{4} \epsilon_{0}, \frac{1}{4} \epsilon_{0}\right) \\
\stackrel{\mathcal{R}_{\rho}}{\longrightarrow} \cdots \stackrel{\mathcal{R}_{\rho}}{\longrightarrow} \mathcal{B}_{0}\left(\sum_{l=1}^{n} \frac{1}{2^{l}} \epsilon_{0}, \frac{1}{2^{n}} \epsilon_{0}, \frac{1}{2^{n}} \epsilon_{0}\right) .
\end{array}
$$

For $w \in \mathcal{B}_{0}\left(\epsilon_{0} / 2, \epsilon_{0} / 2, \epsilon_{0} / 2\right)$ and $n \in \mathbb{N}_{0}$, we define

$$
w^{(n)}:=\mathcal{R}_{\rho}^{n}(w) \in \mathcal{B}_{0}\left(\epsilon_{0}, 2^{-n-1} \epsilon_{0}, 2^{-n-1} \epsilon_{0}\right) .
$$

We introduce the definitions

$$
\begin{aligned}
E_{n, \rho}[w](z) & :=E_{\rho}\left[w^{(n)}\right](z)=-\rho^{-1}\left\langle\Omega, H\left(w^{(n)}(z)\right) \Omega\right\rangle \\
U_{n}[w] & :=U\left[w^{(n)}\right]=\left\{z \in D_{1 / 2}|| E_{n}(z) \mid<\rho / 2\right\} .
\end{aligned}
$$

By Lemma 6.1 the map

$$
J_{n}[w]:=E_{n, \rho}[w]: U_{n}[w] \rightarrow D_{1 / 2}, \quad z \mapsto E_{n, \rho}[w](z)
$$

is an analytic bijection and $J_{n}[w]^{-1}: D_{1 / 2} \rightarrow U_{n}[w] \subset D_{1 / 2}$. For $0 \leq n \leq m$, we define

$$
e_{(n, m)}[w]:=J_{n}[w]^{-1} \circ \cdots \circ J_{m}[w]^{-1}(0) .
$$

Lemma 10.1 stated below immediately implies that the limit $e_{(n, \infty)}[w]:=$ $\lim _{m \rightarrow \infty} e_{(n, m)}[w]$ exists for all $n \in \mathbb{N}_{0}$. 
Lemma 10.1. Assume $(R)$ and let $w \in \mathcal{B}_{0}\left(\epsilon_{0} / 2, \epsilon_{0} / 2, \epsilon_{0} / 2\right)$. Then,

$$
\left|e_{(n, m)}[w]-e_{(n, m+k)}[w]\right| \leq\left(\frac{4 \rho}{3}\right)^{m-n} .
$$

Proof. For notational simplicity we drop the $w$ dependence in the proof. By Lemma 6.1,

$$
\left|\rho \partial_{z} J_{n}(z)-1\right| \leq 1 / 4, \quad \forall z \in U_{n} .
$$

This implies by the inverse function theorem that

$$
\left|\partial_{\zeta} J_{n}^{-1}(\zeta)\right| \leq \frac{4 \rho}{3}, \quad \forall \zeta \in D_{1 / 2} .
$$

An iterated application of (10.4), the convexity of $D_{1 / 2}$, and the chain rule yields

$$
\begin{aligned}
& \left|e_{(n, m)}-e_{(n, m+k)}\right| \\
& \quad=\left|J_{n}^{-1} \circ \cdots \circ J_{m}^{-1}(0)-J_{n}^{-1} \circ \cdots \circ J_{m}^{-1}\left(J_{m+1}^{-1} \circ \cdots \circ J_{k}^{-1}(0)\right)\right| \leq\left(\frac{4 \rho}{3}\right)^{m-n} \frac{1}{2} .
\end{aligned}
$$

Next we introduce some notation. Let

$$
\begin{aligned}
H_{n}[w] & :=H\left(w^{(n)}\left(e_{(n, \infty)}[w]\right)\right) \\
T_{n}[w] & :=w_{0,0}^{(n)}\left(e_{(n, \infty)}[w]\right)\left(H_{f}\right) \\
Q_{n}[w] & :=Q_{\chi_{\rho}}\left(H_{n}[w], T_{n}[w]\right)
\end{aligned}
$$

For $n, m \in \mathbb{N}_{0}$ with $n \leq m$ we define vectors $\psi_{(n, m)}[w] \in \mathcal{H}_{\text {red }}$ by setting $\psi_{(n, n)}[w]=\Omega$ and

$$
\psi_{(n, m)}[w]=Q_{n}[w] \Gamma_{\rho}^{*} Q_{n+1}[w] \Gamma_{\rho}^{*} \cdots Q_{m-1}[w] \Omega .
$$

Lemma 10.1 stated below immediately implies that this sequence converges as $m \rightarrow \infty$, i.e., the limit

$$
\psi_{(n, \infty)}[w]:=\lim _{m \rightarrow \infty} \psi_{(n, m)}[w]
$$

exists for all $n \in \mathbb{N}_{0}$.

Lemma 10.2. Assume $(R)$ and let $w \in \mathcal{B}_{0}\left(\epsilon_{0} / 2, \epsilon_{0} / 2, \epsilon_{0} / 2\right)$. Then

$$
\left\|\psi_{(n, m+1)}[w]-\psi_{(n, m)}[w]\right\| \leq 2^{-m} \frac{16 \epsilon_{0}}{\rho} \exp \left[2^{-n} 32 \epsilon_{0} \rho^{-1}\right] .
$$

Proof. For notational compactness we drop the $w$ dependence in the proof. Note that

$$
\psi_{(n, m+1)}-\psi_{(n, m)}=Q_{n} \Gamma_{\rho}^{*} Q_{n+1} \Gamma_{\rho}^{*} \cdots Q_{m-1} \Gamma_{\rho}^{*}\left(Q_{m}-\chi_{\rho}\right) \Omega,
$$

where we used $\Gamma_{\rho}^{*} \chi_{\rho} \Omega=\Omega$. Next we set $W_{n}:=H_{n}-T_{n}$ and estimate $Q_{n}-\chi_{\rho}$,

$$
\begin{aligned}
\left\|Q_{n}-\chi_{\rho}\right\| & \leq\left\|\bar{\chi}_{\rho}\left(T_{n}+\bar{\chi}_{\rho} W_{n} \bar{\chi}_{\rho}\right)^{-1} \bar{\chi}_{\rho} W_{n} \chi_{\rho}\right\| \\
& \leq\left(\rho / 8-\left\|W_{n}\right\|\right)^{-1}\left\|W_{n}\right\| \\
& \leq \frac{16 \epsilon_{0}}{\rho} 2^{-n}
\end{aligned}
$$


where in the second inequality we used that $\left|T_{n}(r)\right| \geq \rho / 8$ if $r \in\left[\frac{3}{4} \rho, 1\right]$, see (6.4), and in the last inequality we used $\left\|W_{n}\right\| \leq 2^{-n-1} \epsilon_{0} \leq \rho / 16$, see (5.12). Equation (10.8) implies

$$
\left\|Q_{n}\right\| \leq 1+\frac{16 \epsilon_{0}}{\rho} 2^{-n}
$$

Using this and (10.8) to estimate the difference (10.7), we find

$$
\left\|\psi_{(n, m+1)}-\psi_{(n, m)}\right\| \leq 2^{-m} \frac{16 \epsilon_{0}}{\rho} \prod_{j=n}^{m-1}\left[1+2^{-j} 16 \epsilon_{0} / \rho\right] .
$$

The estimate of the lemma follows from $\prod_{j=0}^{\infty}\left(1+\lambda_{j}\right) \leq \exp \left[\sum_{j=0}^{\infty} \lambda_{j}\right]$, which holds for $\lambda_{j} \geq 0$.

We are now ready to state the main theorem of this section.

Theorem 10.3. Assume Hypothesis $(R)$. Let $w \in \mathcal{B}_{0}\left(\epsilon_{0} / 2, \epsilon_{0} / 2, \epsilon_{0} / 2\right)$. Then the complex number $e_{(0, \infty)}[w] \in D_{1 / 2}$ defined in (10.2) is an eigenvalue of $H(w)$, in the sense that

$$
\operatorname{dim} \operatorname{ker}\left\{H\left(w\left(e_{(0, \infty)}[w]\right)\right)\right\} \geq 1 .
$$

Moreover, the vector $\psi_{(0, \infty)}[w]$ defined in $(10.5)$ is a corresponding eigenvector, i.e., is a non-zero element of $\operatorname{ker}\left\{H\left(w\left(e_{(0, \infty)}[w]\right)\right\}\right.$. We have the bound $\left\|\psi_{(0, \infty)}[w]\right\| \leq 4 \mathrm{e}^{4}$. If $w$ is symmetric and $-1 / 2<z<e_{(0, \infty)}[w]$, then $H(w(z))$ is bounded invertible.

Proof. For compactness we suppress the $w$ dependence in the proof. We show that $\psi_{(0, \infty)}$ is a nonzero vector, which is in the kernel of $H\left(w\left(e_{(0, \infty)}\right)\right.$. By (10.6) we have the norm estimate

$$
\left\|\psi_{(n, \infty)}-\Omega\right\|=\left\|\psi_{(n, \infty)}-\psi_{(n, n)}\right\| \leq 2^{-n} \frac{32 \epsilon_{0}}{\rho} \exp \left[2^{-n} 32 \epsilon_{0} \rho^{-1}\right],
$$

This implies that $\psi_{\left(n_{0}, \infty\right)} \neq 0$ provided $n_{0}$ is sufficiently large. Next we show that $\psi_{(n, \infty)}$ is in the kernel of $H_{n}$. To this end, we shall iterate the following identity:

$$
H_{n-1} Q_{n-1} \Gamma_{\rho}^{*}=\rho \Gamma_{\rho}^{*} \chi_{1}\left(H_{f}\right) H_{n},
$$

which is a consequence of Lemma 4.4 of identities involving the Feshbach operator. For $n \leq m$,

$$
\begin{aligned}
H_{n} \psi_{(n, m)} & :=\left(H_{n} Q_{n} \Gamma_{\rho}^{*}\right)\left(Q_{n+1} \Gamma_{\rho}^{*} \cdots Q_{m-1} \Omega\right) \\
& =\rho \Gamma_{\rho}^{*} \chi_{1}\left(H_{n+1} Q_{n+1} \Gamma_{\rho}^{*}\right)\left(Q_{n+2} \Gamma_{\rho}^{*} \cdots Q_{m-1} \Omega\right) \\
& \vdots \\
& =\rho^{m-n}\left(\Gamma_{\rho}^{*} \chi_{1}\right)^{m-n} H_{m} \Omega .
\end{aligned}
$$

Since $H_{n}$ is a bounded operator on $\mathcal{H}_{\text {red }}$ the left-hand side converges to $H_{n} \psi_{(n, \infty)}$ as $m \rightarrow \infty$. Also the right-hand side converges to 0 as $m \rightarrow \infty$, since by (10.1)

$$
\left\|H_{m} \Omega\right\| \leq \text { const. }
$$


and there is an overall factor $\rho^{m-n}$. Thus, taking the limit as $m$ tends to infinity in (10.11) yields for all $n \in \mathbb{N}_{0}$

$$
H_{n} \psi_{(n, \infty)}=0 \text {. }
$$

In particular we have shown, $H_{n_{0}} \psi_{\left(n_{0}, \infty\right)}=0$ and $\psi_{\left(n_{0}, \infty\right)} \neq 0$. A repeated application of the Feshbach property implies that $\psi_{(0, \infty)} \neq 0$ and $H_{0} \psi_{(0, \infty)}=$ 0 . The bound on $\psi_{(0, \infty)}$ follows from Lemma 10.2 and $\epsilon_{0} \leq \rho / 8$.

Now we show the statement about symmetric kernels $w$. Thus let $w$ be symmetric. Then all $w^{(n)}$ are also symmetric, by Theorem 8.2. Let $-\frac{1}{2}<\zeta \leq$ $-\frac{3}{16} \rho$. Then we estimate, with $E_{n}(\zeta):=-\left\langle\Omega, H\left(w^{(n)}(\zeta)\right) \Omega\right\rangle$,

$$
\begin{aligned}
&\left\langle\varphi, H\left(w^{(n)}(\zeta)\right) \varphi\right\rangle \\
&=\left\langle\varphi,\left(T\left[w^{(n)}(\zeta)\right]+E_{n}(\zeta)-\zeta+\zeta-E_{n}(\zeta)+W\left[w^{(n)}(\zeta)\right]\right) \varphi\right\rangle \\
& \geq\left\langle\varphi,\left(T\left[w^{(n)}(\zeta)\right]+E_{n}(\zeta)\right) \varphi\right\rangle-\zeta\|\varphi\|^{2}-\left|\zeta-E_{n}(\zeta)\right|\|\varphi\|^{2} \\
&-\left|\left\langle\varphi, W\left[w^{(n)}(\zeta)\right]\right) \varphi\right\rangle \mid \\
& \geq\left(\frac{3}{16}-\frac{1}{16}-\frac{1}{16}\right) \rho\|\varphi\|^{2}=\frac{1}{16} \rho\|\varphi\|^{2},
\end{aligned}
$$

where the first term in the second line is non-negative since $\left\|\partial_{r} w_{0,0}^{(n)}-1\right\| \leq 1 / 2$ and $w^{(n)}$ is symmetric, and the last term in the second line is estimated using (5.12). Applying Theorem 4.2 iteratively, we find that $H(w(z))$ is bounded invertible if $z \in K_{n}\left(\left(-\frac{1}{2},-\frac{3}{16} \rho\right]\right)$, where we have set $K_{n}:=J_{0}^{-1} \circ \cdots \circ J_{n-1}^{-1}$ if $n \geq 1$ and $K_{0}:=\mathrm{id}$. It follows that $H(w(z))$ is bounded invertible if

$$
z \in I_{N}:=\bigcup_{n=0}^{N} K_{n}\left(\left(-\frac{1}{2},-\frac{3}{16} \rho\right]\right),
$$

for some $N \in \mathbb{N}$. Below we will show that

$$
I_{N} \supset\left(-\frac{1}{2}, K_{N}\left(-\frac{3}{16} \rho\right)\right] .
$$

In view of estimate (10.4) and the definition of $e_{(0, \infty)}$ we have $\lim _{N \rightarrow \infty}$ $K_{N}\left(-\frac{3}{16} \rho\right)=e_{(0, \infty)}$. Thus, $(10.12)$ will imply that $H(w(z))$ is bounded invertible for all $z \in\left(-\frac{1}{2}, e_{(0, \infty)}\right)$. To show $(10.12)$ we first note that $J_{n}^{-1}: D_{1 / 2} \rightarrow U_{n}$ is bijective, differentiable, maps real numbers to real numbers, since $w^{(n)}$ is symmetric, and is increasing because of (10.3). Note that by (10.4) $J_{n}^{-1}$ extends continuously to the boundary of $D_{1 / 2}$. It follows that $U_{n} \cap \mathbb{R}=$ $\left(J_{n}^{-1}(-1 / 2), J_{n}^{-1}(1 / 2)\right)$. Since $D_{\frac{3}{8} \rho} \subset U_{n} \subset D_{1 / 2}$ (see Lemma 6.1) we conclude that

$$
-\frac{1}{2} \leq J_{n}^{-1}(-1 / 2) \leq-\frac{3}{16} \rho .
$$

This implies that for any $b \in(-1 / 2,1 / 2)$, we have

$$
\left(\left(-\frac{1}{2},-\frac{3}{16} \rho\right] \cup J_{n}^{-1}\left(\left(-\frac{1}{2}, b\right]\right)\right) \supset\left(-\frac{1}{2}, J_{n}^{-1}(b)\right] .
$$

Iterating this relation one easily shows (10.12). 
The next theorem states in what sense analytic kernels lead to analytic eigenvalues and eigenvectors. It also relates c-continuous kernels to continuous eigenvalues and eigenvectors.

Theorem 10.4. Assume Hypothesis $(\mathrm{R})$. Let $S$ be an open subset of $\mathbb{C}^{\nu}(a$ topological space). Suppose

$$
\begin{gathered}
w(\cdot, \cdot): S \times D_{1 / 2} \rightarrow \mathcal{W}_{\xi}^{\#} \\
(s, z) \mapsto w(s, z)
\end{gathered}
$$

is an analytic (a c-continuous) function such that $w(s)(\cdot):=w(s, \cdot)$ is in $\mathcal{B}_{0}\left(\epsilon_{0} / 2, \epsilon_{0} / 2, \epsilon_{0} / 2\right)$. Then $s \mapsto e_{(0, \infty)}[w(s)]$ and $s \mapsto \psi_{(0, \infty)}[w(s)]$ are analytic (continuous) functions.

Proof. From Theorems 9.1 and 8.3 it follows that all integral kernels $(s, z) \mapsto$ $w^{(n)}(s, z)$ are analytic (c-continuous). In particular $(s, z) \mapsto E_{\rho}\left[w^{(n)}(s)\right](z)$ is analytic (continuous). It follows that the mapping $(s, z) \mapsto E_{\rho}\left[w^{(n)}(s)\right]^{-1}(z)$ on $S \times D_{1 / 2}$ is analytic (continuous), which can be seen from Lemma 6.1 and identity (8.1). Now it follows from the definition that $e_{(n, m)}[w(s)]$ is an analytic (continuous) function of $s$. By Lemma 10.1 the limits of $e_{(n, m)}[w(s)]$ as $m$ tends to infinity are uniform in $s$. Thus, $s \mapsto e_{(n, \infty)}[w(s)]$ is analytic (continuous). It follows that $H_{n}[w(s)], T_{n}[w(s)]$ depend analytically on $s$ by the inequality (5.11) (continuously on $s$ by Lemma 5.7). This implies that $Q_{n}[w(s)]$ is an analytic (continuous) function of $s$. By definition now also $\psi_{(n, m)}[w(s)]$ is an analytic (continuous) function of $s$. Since by Lemma 10.2 the limit of $\psi_{(n, m)}[w(s)]$ as $m$ tends to infinity is uniform in $s$, it follows that $\psi_{(0, \infty)}[w(s)]$ is also an analytic (continuous) function of $s$.

\section{Initial Feshbach Transformations}

We perform two initial Feshbach transformations before we start the renormalization procedure. Specifically, the first application of the Feshbach transformation yields the operator defined in (11.1), where the definition is justified by Theorem 11.1. A second application of the Feshbach transformation yields the operator defined in (11.6), where the definition is justified by Theorem 11.2. The main theorem of this section is Theorem 11.3. It states that the second Feshbach map (11.6) can be expressed in terms of an integral kernel, (11.11), which satisfies suitable analyticity and continuity properties. The proof of Theorem 11.3 relies on estimating each term in a Neumann expansion.

Throughout this section we will assume that Hypothesis $(\mathrm{H})$ holds. First we set

$$
\chi^{(I)}=P_{1} \otimes 1, \quad \bar{\chi}^{(I)}=P_{2} \otimes 1
$$

where

$$
P_{1}=\left(\begin{array}{ll}
0 & 0 \\
0 & 1
\end{array}\right), \quad P_{2}=\left(\begin{array}{ll}
1 & 0 \\
0 & 0
\end{array}\right)
$$


We do not choose to include a boson momentum cutoff in $\chi^{(I)}$, since the associated Feshbach map would otherwise contain terms which are linear in creation and annihilation operators.

Theorem 11.1. $\left(H_{\lambda, \sigma}-z, H_{f}+\tau-z\right)$ is a Feshbach pair for $\chi^{(I)}$ provided $|z|<2$.

Proof. We will identify the ranges of $P_{1}$ and $P_{2}$ with $\mathcal{F}$. If is sufficient to verify the assumptions of Lemma 4.3. These are easily verified noting that $\left.\left(H_{f}+\tau-z\right)\right|_{\operatorname{Ran} \bar{\chi}^{(I)}} \cong H_{f}+2-z$ is invertible if $|z|<2, P_{2} \sigma_{x} \phi\left(f_{\sigma}\right) P_{2}=0$, and by elementary estimates given in Appendix A we have that $\left(H_{f}+2-z\right)^{-1} \phi\left(f_{\sigma}\right)$ is bounded if $|z|<2$

By Theorem 11.1 the following definition makes sense for $|z|<2$ :

$$
\begin{aligned}
H_{\lambda, \sigma}^{(I)}(z) & :=F_{\chi^{(I)}}\left(H_{\lambda, \sigma}, H_{f}+\tau-z\right) \uparrow \operatorname{Ran} \chi^{(I)} \\
& \cong H_{f}-z-\lambda^{2} \phi\left(f_{\sigma}\right)\left(H_{f}+2-z\right)^{-1} \phi\left(f_{\sigma}\right),
\end{aligned}
$$

where we identified the range of $\chi^{(I)}$ with the Fock space. Next we use the pullthrough formula, Lemma (A.1), to express $H_{\lambda, \sigma}^{(I)}$ in terms of integral kernels. To this end we introduce the notation

$$
\bar{H}_{m, n}\left(w_{m, n}\right)=\int_{\mathbb{R}^{m+n}} \frac{\mathrm{d} K^{(m, n)}}{\left|K^{(m, n)}\right|^{1 / 2}} a^{*}\left(k^{(m)}\right) w_{m, n}\left(H_{f}, K^{(m, n)}\right) a\left(\tilde{k}^{(n)}\right) .
$$

Definition (11.2) is understood in the sense of forms. We have

$$
H_{\lambda, \sigma}^{(I)}=T_{\lambda, \sigma}^{(I)}+W_{\lambda, \sigma}^{(I)}
$$

with $T_{\lambda, \sigma}^{(I)}(z)=\bar{H}_{0,0}\left(w_{0,0}^{(I)}(\lambda, \sigma, z)\right)$ and $W_{\lambda, \sigma}^{(I)}(z):=\sum_{m+n=2} \bar{H}_{m, n}\left(w^{(I)}(\lambda, \sigma, z)\right)$ where

$$
t^{(I)}(\lambda, \sigma, z)(r):=w_{0,0}^{(I)}(\lambda, \sigma, z)(r):=r-z-\lambda^{2} \int \frac{\mathrm{d}^{3} k}{(4 \pi)^{2} \omega(k)} \frac{\left|f_{\sigma}(k)\right|^{2}}{r+|k|+2-z},
$$

and $w_{m, n}^{(I)}=\left(\widehat{w}_{m, n}^{(I)}\right)^{(\mathrm{sym})}$, with

$$
\begin{aligned}
& \widehat{w}_{2,0}^{(I)}(\lambda, \sigma, z)\left(r, k_{1}, k_{2}\right):=-\lambda^{2} f_{\sigma}\left(k_{1}\right) f_{\sigma}\left(k_{2}\right) \frac{1}{r+\left|k_{1}\right|+2-z}, \\
& \widehat{w}_{0,2}^{(I)}(\lambda, \sigma, z)\left(r, \widetilde{k}_{1}, \widetilde{k}_{2}\right):=-\lambda^{2} \frac{1}{r+\left|\widetilde{k}_{1}\right|+2-z} \overline{f_{\sigma}}\left(\widetilde{k}_{1}\right) \bar{f}_{\sigma}\left(\widetilde{k}_{2}\right), \\
& \widehat{w}_{1,1}^{(I)}(\lambda, \sigma, z)\left(r, k_{1}, \widetilde{k}_{1}\right):=-\lambda^{2} f_{\sigma}\left(k_{1}\right)\left[\frac{1}{r+2-z}+\frac{1}{r+\left|k_{1}\right|+\left|\widetilde{k}_{1}\right|+2-z}\right] \\
& \times \bar{f}_{\sigma}\left(\widetilde{k}_{1}\right) .
\end{aligned}
$$

By $w^{(I)}$ we denote the tuple consisting of the four components $w_{m, n}^{(I)}$ with $m+n=0,2$. We will now apply the Feshbach transformation one more time. 
The next theorem states that for sufficiently small values of the coupling constant $\left(H_{\lambda, \sigma}^{(I)}, T_{\lambda, \sigma}^{(I)}\right)$ is a Feshbach pair for $\chi_{1}$. To formulate the theorem we introduce the following constant:

$$
\mu_{0}:=(8 \max (\|f /(4 \pi \sqrt{\omega})\|,\|f /(4 \pi \omega)\|))^{-1} .
$$

Theorem 11.2. Let $|\lambda|<\mu_{0}, \sigma \geq 0$, and $|z| \leq 1 / 2$. Then the pair of operators $\left(H_{\lambda, \sigma}^{(I)}(z), T_{\lambda, \sigma}^{(I)}(z)\right)$ is a Feshbach pair for $\chi_{1}$ and on $D_{1 / 2}$ we have

$$
\left\|\left.T_{\lambda, \sigma}^{(I)}{ }^{-1}\right|_{\operatorname{Ran} \bar{\chi}_{1}}\right\| \leq \frac{64}{15}, \quad\left\|T_{\lambda, \sigma}^{(I)}{ }^{-1} \bar{\chi}_{1} W_{\lambda, \sigma}^{(I)}\right\|<\frac{7}{15}, \quad\left\|W_{\lambda, \sigma}^{(I)} T_{\lambda, \sigma}^{(I)}{ }^{-1} \bar{\chi}_{1}\right\|<\frac{7}{15} .
$$

Proof. Let $\delta_{0}:=\max (\|f /(4 \pi \sqrt{\omega})\|,\|f /(4 \pi \omega)\|)$. First we show that on $D_{1 / 2}$ we have

$$
\left\|W_{\lambda, \sigma}^{(I)}\right\| \leq|\lambda|^{2} 7 \delta_{0}^{2}
$$

To this end note that

$$
W_{\lambda, \sigma}^{(I)}(z)=-\lambda^{2} \phi\left(f_{\sigma}\right)\left(H_{f}+2-z\right)^{-1} \phi\left(f_{\sigma}\right)+\lambda^{2} \int \frac{\mathrm{d}^{3} k}{(4 \pi)^{2} \omega(k)} \frac{\left|f_{\sigma}(k)\right|^{2}}{H_{f}+|k|+2-z} .
$$

For $|z| \leq 1 / 2$, this yields the estimate

$$
\left\|W_{\lambda, \sigma}^{(I)}\right\| \leq|\lambda|^{2}\left\|\phi\left(f_{\sigma}\right)\left(H_{f}+1\right)^{-1 / 2}\right\|\left\|\left(H_{f}+1\right)^{-1 / 2} \phi\left(f_{\sigma}\right)\right\|+|\lambda|^{2}\|f /(4 \pi \sqrt{\omega})\|^{2} .
$$

Now using elementary estimates collected in Lemma A.6 to estimate the first term in (11.5) one obtains (11.4). Next observe that $T_{\lambda, \sigma}^{(I)}$ commutes with $\chi_{1}$ and $\bar{\chi}_{1}$. From the following estimate it follows that $T_{\lambda, \sigma}^{(I)}$ is bounded invertible on the range of $\bar{\chi}_{1}$. For $r \geq 3 / 4$,

$$
\left|t^{(I)}(\lambda, \sigma, z)(r)\right| \geq 3 / 4-1 / 2-|\lambda|^{2} \int \frac{\mathrm{d}^{3} k}{(4 \pi)^{2} \omega(k)} \frac{|f(k)|^{2}}{|k|+1} \geq 15 / 64 .
$$

This and (11.4) imply (11.3). In view of Lemma 4.3 it follows that $\left(H_{\lambda, \sigma}^{(I)}, T_{\lambda, \sigma}^{(I)}\right)$ is a Feshbach pair for $\chi_{1}$.

Let $|\lambda|<\mu_{0}$ and $|z| \leq 1 / 2$. Then by Theorem 11.2 the second Feshbach map,

$$
H_{\lambda, \sigma}^{(0)}(z):=F_{\chi_{1}}\left(H_{\lambda, \sigma}^{(I)}(z), T_{\lambda, \sigma}^{(I)}(z)\right) \uparrow \mathcal{H}_{\mathrm{red}}
$$

is well defined and we are allowed to expand the operator $H_{\lambda, \sigma}^{(0)}(z)$ in a Neumann series. We obtain on $\mathcal{H}_{\text {red }}$

$$
\begin{aligned}
H^{(0)}= & T^{(I)}+\chi_{1} W^{(I)} \chi_{1}-\chi_{1} W^{(I)} \bar{\chi}_{1} \\
& \times \sum_{n=0}^{\infty}\left(-T^{(I)^{-1}} \bar{\chi}_{1} W^{(I)} \bar{\chi}_{1}\right)^{n} T^{(I)}{ }^{-1} \bar{\chi}_{1} W^{(I)} \chi_{1},
\end{aligned}
$$

where we dropped the $\lambda, \sigma, z$ dependence and assumed that $z \in D_{1 / 2}$. Again we normal order the above expression, using the pull-through formula. To this 
end we use the identity of Theorem 7.2, which also holds for the integral kernels considered here since its proof is based on algebraic identities. This yields a sequence of integral kernels $\widetilde{w}^{(0)}$, which are given as follows: For $M+N \geq 1$,

$$
\begin{aligned}
& \widetilde{w}_{M, N}^{(0)}(\lambda, \sigma, z)\left(r, K^{(M, N)}\right)=\sum_{L=1}^{\infty}(-1)^{L+1} \sum_{\substack{(\underline{m}, \underline{p}, \underline{n}, \underline{q}) \in \mathbb{N}_{0}^{4 L}: \\
|\underline{m}|=M,|\underline{n}|=N, m l+p_{l}+q_{l}+n_{l}=2}} \\
& \quad \times \prod_{l=1}^{L}\left\{\left(\begin{array}{c}
m_{l}+p_{l} \\
p_{l}
\end{array}\right)\left(\begin{array}{c}
n_{l}+q_{l} \\
q_{l}
\end{array}\right)\right\} V_{(\underline{m}, \underline{p}, \underline{n}, \underline{q})}\left[w^{(I)}(\lambda, \sigma, z)\right]\left(r, K^{(M, N)}\right) .
\end{aligned}
$$

Furthermore,

$$
\begin{aligned}
& \widetilde{w}_{0,0}^{(0)}(\lambda, \sigma, z)(r) \\
& \quad=t^{(I)}(\lambda, \sigma, z)(r)+\sum_{L=2}^{\infty}(-1)^{L+1} \sum_{(\underline{p}, \underline{q}) \in \mathbb{N}_{0}^{2 L}: p_{l}+q_{l}=2} V_{(\underline{0}, \underline{p}, \underline{0}, \underline{q})}\left[w^{(I)}(\lambda, \sigma, z)\right](r),
\end{aligned}
$$

where for $(\underline{m}, \underline{p}, \underline{n}, \underline{q}) \in \mathbb{N}_{0}^{4 L}$ we have used the definition

$$
\begin{aligned}
V_{\underline{m}, \underline{p}, \underline{n}, \underline{q}}[w]\left(r, K^{(|\underline{m}|,|\underline{n}|)}\right) \\
\quad:=\left\langle\Omega, \bar{F}_{0}\left(H_{f}+r\right) \prod_{l=1}^{L}\left\{\bar{W}_{p_{l}, q_{l}}^{m_{l}, n_{l}}[w]\left(r+r_{l}, K^{\left(m_{l}, n_{l}\right)}\right) \bar{F}_{l}\left(H_{f}+r+\widetilde{r}_{l}\right)\right\} \Omega\right\rangle
\end{aligned}
$$

with $\bar{F}_{0}[w](r):=\chi_{1}(r), \bar{F}_{L}[w](r):=\chi_{1}(r)$, and for $l=1, \ldots, L-1$ we set

$$
\bar{F}_{l}[w](r):=\bar{F}[w](r):=\frac{\bar{\chi}_{1}(r)^{2}}{w_{0,0}(r)} .
$$

Here, we used the definition

$$
\begin{aligned}
& \bar{W}_{p, q}^{m, n}[w]\left(r, K^{(m, n)}\right] \\
& \quad:=\int \frac{\mathrm{d} X^{(p, q)}}{\mid X^{(p, q) \mid 1 / 2}} a^{*}\left(x^{(p)}\right) w_{m+p, n+q}\left(H_{f}+r, k^{(m)}, x^{(p)}, \widetilde{k}^{(n)}, \widetilde{x}^{(q)}\right) a\left(\widetilde{x}^{(q)}\right) .
\end{aligned}
$$

Recall also the notation introduced in Theorem 7.2. Since we want to consider symmetric kernels we set $w^{(0)}:=\left(\widetilde{w}^{(0)}\right)^{(\text {sym })}$. We are now ready to state the main theorem of this section.

Theorem 11.3. Let $0<\xi<1$ and $\delta_{1}, \delta_{2}, \delta_{3}>0$. Then, there exists a positive $\lambda_{0} \leq \mu_{0}$ such that for all $\lambda \in D_{\lambda_{0}}$ and $\sigma \geq 0$ we have

$$
\begin{gathered}
w^{(0)}(\lambda, \sigma, \cdot) \in \mathcal{B}_{0}\left(\delta_{1}, \delta_{2}, \delta_{2}\right) \\
H_{\lambda, \sigma}^{(0)}(z)=H\left(w^{(0)}(\lambda, \sigma, z)\right), \quad \forall z \in D_{1 / 2} .
\end{gathered}
$$

Moreover the following is true: 
(i) For $\sigma \geq 0$, the map $(\lambda, z) \mapsto w^{(0)}(\lambda, \sigma, z)$ is a $\mathcal{W}_{\xi}^{\#}$-valued analytic function on $D_{\lambda_{0}} \times D_{1 / 2}$.

(ii) For each $\lambda \in D_{\lambda_{0}}$, the $\operatorname{map}(\sigma, z) \mapsto w^{(0)}(\lambda, \sigma, z) \in \mathcal{W}_{\xi}^{\#}$ is a c-continuous function on $[0, \infty) \times D_{1 / 2}$.

(iii) For real $\lambda \in D_{\lambda_{0}}$ and $\sigma \geq 0$, the kernel $w^{(0)}(\lambda, \sigma)$ is symmetric.

The remaining part of this section is devoted to the proof of Theorem 11.3. Let us first outline the proof. From the previous discussion we know that once (11.10) has been established then (11.11) will follow. Thus first we will show (11.10). The fact that the number of creation and annihilation operators of $w^{(0)}$ is even, follows directly from the definition. Showing (11.10) also requires an estimate of the kernel. To this end we use an estimate on $V_{\underline{m}, \underline{p}, \underline{n}, \underline{q}}\left[w^{(I)}\right]$ which is given in Lemma 11.4, below. Using that estimate for $V_{\underline{m}, \underline{p}, \underline{n}, \underline{q}}\left[w^{(I)}\right]$, we will then obtain estimates $(11.17),(11.18)$, and (11.19), which imply (11.10). Those estimates establish uniform convergence which will then be used to show (i) and (ii) using the corresponding statement for $V_{\underline{m}, \underline{p}, \underline{n}, \underline{q}}\left[w^{(I)}\right]$. (iii) follows from the definition and (5.14).

First we show the following Lemma:

Lemma 11.4. Let $|z| \leq 1 / 2,|\lambda| \leq \mu_{0}$, and $\sigma \geq 0$. Then

$$
\left\|V_{\underline{m}, \underline{p}, \underline{n}, \underline{q}}\left[w^{(I)}(\lambda, \sigma, z)\right]\right\|^{\#} \leq(L+1) C_{\bar{F}}^{L+1} C_{W}(\lambda)^{L} .
$$

where

$$
\begin{aligned}
C_{\bar{F}} & :=10\left\|\partial_{r} \bar{\chi}_{1}\right\|_{\infty}+31 \\
C_{W}(\lambda) & :=\sup _{m+n+p+q=2}|\lambda|^{2} 6\left(\|f / \omega\|^{2}+2\|f\|\|f / \omega\|\right)^{\frac{p+q}{2}}\|f\|_{\infty}^{m+n} .
\end{aligned}
$$

Lemma 11.4 will essentially follow from Lemma 11.5, shown below. To this end we introduce the following norms. Again we will use the canonical identification of the space $L^{\infty}\left(\mathbb{R}^{3 m+3 n} ; C[0, \infty)\right)$ with a subset of $L^{\infty}([0, \infty) \times$ $\left.\mathbb{R}^{3 m+3 n}\right)$. For functions $w_{m, n} \in L^{\infty}\left(\mathbb{R}^{3 m+3 n} ; C[0, \infty)\right)$ and any $(s, p, t, q)$ with $m=s+p$ and $n=t+q$ we define the norm

$$
\begin{aligned}
\|w\|_{s, p, t, q}^{b}:= & \operatorname{essip}_{\left(k^{(s)}, \widetilde{k}^{(t)}\right) \in \mathbb{R}^{3 s+3 t}}\left[\int \frac{\mathrm{d} X^{(p, q)}}{\left|X^{(p, q)}\right|^{2}}\right. \\
& \left.\times \sup _{r \geq 0}\left|w_{m, n}\left(r, k^{(s)}, x^{(p)}, \widetilde{k}^{(t)}, \widetilde{x}^{(q)}\right)\right|^{2}\left(r+\Sigma\left[x^{(p)}\right]\right)^{p}\left(r+\Sigma\left[\widetilde{x}^{(q)}\right]\right)^{q}\right]^{1 / 2} .
\end{aligned}
$$

Using Lemma A.5 in Appendix A, we see that

$$
\left\|\bar{W}_{p, q}^{m, n}[w]\left(r, K^{(m, n)}\right)\right\|_{\mathrm{op}} \leq\left\|w_{m+p, n+q}\right\|_{m, p, n, q}^{b}
$$

and if $w_{m, n} \in L^{\infty}\left(B_{1}^{m+n} ; C^{1}[0, \infty)\right)$ we have

$$
\left\|\partial_{r} \bar{W}_{p, q}^{m, n}[w]\left(r, K^{(m, n)}\right)\right\|_{\mathrm{op}} \leq\left\|\partial_{r} w_{m+p, n+q}\right\|_{m, p, n, q}^{b},
$$

where the partial derivative $\partial_{r} \bar{W}_{p, q}^{m, n}[w]$ is understood with respect to the weak operator topology. 
Lemma 11.5. Let $|z| \leq 1 / 2$ and $\sigma \geq 0$. Then the following statements are true:

(a) If $m+n+p+q=2$ and $s=0,1$, then we have

$$
\begin{aligned}
& \left\|\partial_{r}^{s} W_{p, q}^{m, n}\left[w^{(I)}(\lambda, \sigma, z)\right]\left(r, K^{(m, n)}\right)\right\|_{\mathrm{op}} \\
& \quad \leq|\lambda|^{2} 6\left(\|f / \omega\|^{2}+2\|f\|\|f / \omega\|\right)^{\frac{p+q}{2}}\|f\|_{\infty}^{m+n} .
\end{aligned}
$$

(b) Let $|\lambda| \leq \mu_{0}$. Then

$$
\begin{aligned}
& \left\|\left(\partial_{r} \bar{F}\left[w^{(I)}(\lambda, \sigma, z)\right]\right)\left(H_{f}+r\right)\right\|+\left\|\bar{F}\left[w^{(I)}(\lambda, \sigma, z)\right]\left(H_{f}+r\right)\right\| \\
& \quad \leq 10\left\|\partial_{r} \bar{\chi}_{1}\right\|_{\infty}+31 .
\end{aligned}
$$

Proof. (a) In view of inequalities (11.13) and (11.14) we need to estimate the following, where we use the abbreviation $f\left(x^{(p)}\right)=f\left(x_{1}\right) \cdots f\left(x_{p}\right)$ :

$$
\begin{aligned}
& {\left[\left\|\partial_{r}^{s} w_{m+p, n+q}^{(I)}(\lambda, \sigma, z)\right\|_{m, p, n, q}^{b}\right]^{2}} \\
& \quad \leq|\lambda|^{2}\|f\|_{\infty}^{m+n} 4 \int \frac{\mathrm{d} X^{(p, q)}}{\left|X^{(p, q)}\right|^{2}} \sup _{r \geq 0} \frac{\left|f\left(x^{(p)}\right)\right|^{2}\left|f\left(\widetilde{x}^{(q)}\right)\right|^{2}}{|r+1|^{2+s}} \\
& \quad \times\left(r+\Sigma\left[x^{(p)}\right]\right)^{p}\left(r+\Sigma\left[\widetilde{x}^{(q)}\right]\right)^{q} \\
& \quad \leq|\lambda|^{2} 4\|f\|_{\infty}^{m+n} E_{p}(f) E_{q}(f),
\end{aligned}
$$

where

$$
E_{p}(f):=\left.\int_{\mathbb{R}^{p}} \frac{\mathrm{d} x^{(p)}}{\left|x^{(p)}\right|^{2}}|| f\left(x^{(p)}\right)\right|^{2}\left(1+\Sigma\left[x^{(p)}\right]\right)^{p} .
$$

we have $E_{0}(f)=1$ and for $p=1,2$ it is an elementary estimate to obtain $E_{p}(f) \leq 3\left(\|f / \omega\|^{2}+2\|f\|\|f / \omega\|\right)^{p}$.

(b) We have using Lemma 11.2

$$
\left\|\bar{F}\left[w^{(I)}(\lambda, \sigma, z)\right]\left(H_{f}+r\right)\right\| \leq\left[\inf _{r \geq 3 / 4} t^{(I)}(\lambda, \sigma, z)(r)\right]^{-1} \leq 64 / 15 .
$$

Similarly, we estimate the derivative. First we have

$$
\partial_{r} \bar{F}\left[w^{(I)}\right]=\frac{2 \bar{\chi}_{1} \partial_{r} \bar{\chi}_{1}}{t^{(I)}}-\frac{\bar{\chi}_{1}^{2} \partial_{r} t^{(I)}}{\left(t^{(I)}\right)^{2}} .
$$

Now

$$
\partial_{r} t^{(I)}(\lambda, \sigma, z)(r)=1+\lambda^{2} \int \frac{\mathrm{d}^{3} k}{(4 \pi)^{2} \omega(k)} \frac{\left|f_{\sigma}(k)\right|^{2}}{(r+|k|+2-z)^{2}} .
$$

This yields the estimate $\left|\partial_{r} t^{(I)}(\lambda, \sigma, z)(r)\right| \leq 1+|\lambda|^{2}\|f /(4 \pi \sqrt{\omega})\|^{2} \leq 1+1 / 64$. Thus, we find using again Lemma 11.2, $\left\|\partial_{r} \bar{F}\left(H_{f}+r\right)\right\| \leq 10\left\|\partial_{r} \bar{\chi}_{1}\right\|_{\infty}+26$, and hence (b) follows.

Proof of Lemma 11.4. First observe that $V_{\underline{m}, \underline{p}, \underline{n}, \underline{q}}\left[w^{(I)}\right]$ satisfies the property (i) of the definition of $\mathcal{W}_{|\underline{m}|,|\underline{n}|}^{\#}$. To estimate the norm $\left\|V_{\underline{m}, \underline{p}, \underline{n}, \underline{q}}\left[w^{(I)}\right]\right\| \|^{\#}$ we use (7.3), the estimates of Lemma 11.5, and we calculate the derivative with respect to $r$ using Leibniz' rule. 
Using Lemma 11.4 we are now ready to show (11.10). To this end we let $S_{M, N}^{L}$ denote the set of tuples $(\underline{m}, p, \underline{n}, q) \in \mathbb{N}_{0}^{4 L}$ with $|\underline{m}|=M,|\underline{n}|=N$, and $m_{l}+p_{l}+q_{l}+n_{l}=2$. We estimate the combinatorial factor in (11.7) by $2^{L}$ and obtain for $z \in D_{1 / 2}$

$$
\begin{aligned}
\left\|w_{\geq 1}^{(0)}(\lambda, \sigma, z)\right\|^{\#} & =\sum_{M+N \geq 1} \xi^{-(M+N)}\left\|\widetilde{w}_{M, N}(\lambda, \sigma, z)\right\|^{\#} \\
& \leq \sum_{M+N \geq 1} \sum_{L=1}^{\infty} \sum_{(\underline{m}, \underline{p}, \underline{n}, \underline{q}) \in S_{M, N}^{L}} \xi^{-(M+N)} 2^{L} \| V_{\underline{m}, \underline{p}, \underline{n}, \underline{q}\left[w^{(I)}(\lambda, \sigma, z)\right] \|^{\#}} \\
& \leq \sum_{L=1}^{\infty} \sum_{M+N \geq 1} \sum_{(\underline{m}, \underline{p}, \underline{n}, \underline{q}) \in S_{M, N}^{L}} \xi^{-|\underline{m}|-|\underline{n}|}(L+1) C_{\bar{F}}\left[2 C_{W}(\lambda) C_{\bar{F}}\right]^{L} \\
& \leq \sum_{L=1}^{\infty}(L+1) 10^{L} \xi^{-2 L} C_{\bar{F}}\left[2 C_{W}(\lambda) C_{\bar{F}}\right]^{L},
\end{aligned}
$$

where we used (11.12) in the first inequality, and in the second inequality we used $|\underline{m}|+|\underline{n}| \leq 2 L$ and that the number of elements $(\underline{m}, \underline{p}, \underline{n}, \underline{q}) \in \mathbb{N}_{0}^{L}$ with $m_{l}+n_{l}+p_{l}+q_{l}=2$ is bounded by $10^{L}$. A similar but simpler estimate, yields

$$
\begin{aligned}
& \left\|\partial_{r} w_{0,0}^{(0)}(\lambda, \sigma, z)-1\right\| \\
& \quad \leq\left\|\partial_{r} t^{(I)}(\lambda, \sigma, z)-1\right\|+\sum_{L=2}^{\infty} \sum_{(\underline{p}, \underline{q}) \in \mathbb{N}_{0}^{2 L}: p_{l}+q_{l}=2}\left\|V_{\underline{0}, \underline{p}, \underline{0}, \underline{q}}\left[w^{(I)}(\lambda, \sigma, z)\right]\right\|^{\#} \\
& \quad \leq|\lambda|^{2}\|f /(4 \pi \sqrt{\omega})\|^{2}+\sum_{L=2}^{\infty} 3^{L}(L+1) C_{\bar{F}}\left[C_{W}(\lambda) C_{\bar{F}}\right]^{L} .
\end{aligned}
$$

Analogously, we have

$$
\begin{aligned}
& \left\|w_{0,0}^{(0)}(\lambda, \sigma, z)(0)-z\right\| \\
& \quad \leq\left\|t^{(I)}(\lambda, \sigma, z)(0)-z\right\|+\sum_{L=2}^{\infty} \sum_{(\underline{p}, \underline{q}) \in \mathbb{N}_{0}^{2 L}: p_{l}+q_{l}=2}\left\|V_{\underline{0}, \underline{p}, \underline{0}, \underline{q}}\left[w^{(I)}(\lambda, \sigma, z)\right]\right\|^{\#} \\
& \quad \leq|\lambda|^{2}\|f /(4 \pi \sqrt{\omega})\|^{2}+\sum_{L=2}^{\infty} 3^{L}(L+1) C_{\bar{F}}\left[C_{W}(\lambda) C_{\bar{F}}\right]^{L}
\end{aligned}
$$

In view of the definition of $C_{W}(\lambda)$ the right-hand side in (11.17)-(11.19) can be made arbitrarily small for sufficiently small $|\lambda|$. It now remains to show (i) and (ii) of Theorem 11.3.

Part (i) follows from the convergence established in estimates (11.17)(11.19), which is uniform in $(\lambda, z) \in D_{\lambda_{0}} \times D_{1 / 2}$ for some ball $D_{\lambda_{0}}$ of nonzero radius, and the following lemma:

Lemma 11.6. For $(\underline{m}, \underline{p}, \underline{n}, \underline{q}) \in \mathbb{N}_{0}^{4 L}$ and $\sigma \geq 0$, the function

$$
(\lambda, z) \mapsto V_{\underline{m}, \underline{p}, \underline{n}, \underline{q}}\left[w^{(I)}(\lambda, \sigma, z)\right]
$$

is an analytic $\mathcal{W}_{|\underline{m}|,|\underline{n}|}^{\#}$-valued function on $D_{\mu_{0}} \times D_{1 / 2}$. 
Proof. The idea of the proof is to show that (11.20) is a composition of an analytic map with an F-differentiable mapping between suitable Banach spaces. The lemma will follow from Steps 1 and 2, below. First we introduce the following Banach spaces: Let

$$
\overline{\mathcal{W}}_{0,0}^{\#}:=\left\{w \in C^{1}[0, \infty) \mid\|w\|^{\#}:=\|w\|_{\infty}+\left\|\partial_{r} w\right\|_{\infty}\right\} .
$$

Let $\overline{\mathcal{W}}_{m, n}^{\#}$ be the Banach space consisting of functions $w_{m, n} \in L^{\infty}\left(\mathbb{R}^{3 m+3 n}\right.$; $\left.C^{1}[0, \infty)\right)$ satisfying the following properties:

(a) $w_{m, n}=w_{m, n}^{(\mathrm{sym})}$,

(b) the following norm is finite

$$
\left\|w_{m, n}\right\|^{\#}:=\sup _{\substack{s+p=m \\ t+q=n}}\left\|w_{m, n}\right\|_{s, p, t, q}^{b}+\sup _{\substack{s+p=m \\ t+q=n}}\left\|\partial_{r} w_{m, n}\right\|_{s, p, t, q}^{b} .
$$

Hence for almost all $K^{(m, n)} \in \mathbb{R}^{3 m+3 n}$ we have $w_{m, n}\left(\cdot, K^{(m, n)}\right) \in C^{1}[0, \infty)$, where the derivative is denoted by $\partial_{r} w_{m, n}$. Furthermore, we introduce the Banach space $\overline{\mathcal{W}}^{\#}:=\overline{\mathcal{W}}_{0,0}^{\#} \oplus \overline{\mathcal{W}}_{0,2}^{\#} \oplus \overline{\mathcal{W}}_{2,0}^{\#} \oplus \overline{\mathcal{W}}_{1,1}^{\#}$ with norm $\|w\|:=$ $\sum_{m+n=0,2}\left\|w_{m, n}\right\|^{\#}$.

Step 1: Let $\epsilon>0$. Then the map on $\overline{\mathcal{O}}^{(\epsilon)}:=\left\{w \in \overline{\mathcal{W}}^{\#}\left|\inf _{r \geq \frac{3}{4}}\right| w_{0,0}(r) \mid>\epsilon\right\}$

$$
\begin{aligned}
V_{\underline{m}, \underline{p}, \underline{n}, \underline{q}}[\cdot]: \overline{\mathcal{O}}^{(\epsilon)} & \rightarrow \mathcal{W}_{|\underline{m}|,|\underline{n}|}^{\#} \\
w & \mapsto V_{\underline{m}, \underline{p}, \underline{n}, \underline{q}}[w]
\end{aligned}
$$

is F-differentiable.

Step 1 is shown by writing $V_{\underline{m}, p, \underline{n}, q}[\cdot]$ as a composition of the F-differentiable maps defined in (11.21) and (11.23). Let $\underline{w}=\left(w_{1}, \ldots, w_{L}\right)$ with $w_{l} \in \overline{\mathcal{W}}_{n_{l}, m_{l}}$ and $\underline{G}=\left(G_{1}, \ldots, G_{L}\right)$ with $G_{l} \in \overline{\mathcal{W}}_{0,0}$. Then, the expression

$$
\begin{aligned}
& \widetilde{\widetilde{V}}_{\underline{m}, \underline{p}, \underline{n}, \underline{q}}[\underline{w}, \underline{G}]\left(r, K^{(|\underline{m}|,|\underline{n}|))}\right. \\
& \quad:=\left\langle\Omega, G_{0}\left(r+\widetilde{r}_{0}\right) \prod_{l=1}^{L}\left\{\bar{W}_{p_{l}, q_{l}}^{m_{l}, n_{l}}\left[w_{l}\right]\left(r+r_{l}, K_{l}^{\left(m_{l}, n_{l}\right)}\right) G_{l}\left(r+\widetilde{r}_{l}\right)\right\} \Omega\right\rangle,
\end{aligned}
$$

satisfies the property (a) of the definition of $\mathcal{W}_{\underline{m}, \underline{n}}^{\#}$ if $G_{0}$ and $G_{L}$ have support contained in $[0,1)$. Moreover, one easily shows the bound

$$
\left\|\widetilde{V}_{\underline{m}, \underline{p}, \underline{n}, \underline{q}}[\underline{w}, \underline{G}]\right\|^{\#} \leq \prod_{l=0}^{L}\left\{\left\|G_{l}\right\|^{\#}\right\} \prod_{l=1}^{L}\left\{\left\|w_{l}\right\|^{\#}\right\}
$$

calculating the derivative $\partial_{r} \widetilde{V}_{\underline{m}, \underline{p}, \underline{n}, \underline{q}}[\underline{w}, \underline{G}]$ using Leibniz' rule and estimating the resulting expression using inequality (7.3) and estimates (11.13 and (11.14). To show Step 1 it remains to observe that the map on $\overline{\mathcal{O}}_{0,0}^{(\epsilon)}:=$ $\left\{t \in \overline{\mathcal{W}}_{0,0}^{\#}\left|\inf _{r \geq \frac{3}{4}}\right| w_{0,0}(r) \mid>\epsilon\right\}$ 


$$
\bar{F}[\cdot]: \overline{\mathcal{O}}_{0,0}^{(\epsilon)} \longrightarrow \overline{\mathcal{W}}_{0,0}^{\#}, \quad t \mapsto \frac{\bar{\chi}_{1}^{2}}{t}
$$

is F-differentiable. Now this is proved essentially the same way as the first part of Lemma 8.6.

Step 2: The function $(\lambda, z) \mapsto w^{(I)}(\lambda, \sigma, z)$ is an analytic $\overline{\mathcal{W}}^{\#}$-valued function on $D_{\mu_{0}} \times D_{1 / 2}$.

Let $(\lambda, z) \in D_{\mu_{0}} \times D_{1 / 2}$. First observe that $w^{(I)}(\lambda, \sigma, z) \in \overline{\mathcal{W}}^{\#}$, which follows from inequalities (11.15) and (11.16). Analyticity in $\lambda$ is trivial since $w^{(I)}$ is a polynomial of second degree in $\lambda$, with coefficients which are elements of $\overline{\mathcal{W}}^{\#}$, which again follows from inequality 11.16 and estimates used to show (11.15). To show analyticity in $z$ we show the following estimate for $\lambda \in D_{\lambda_{0}}$ and $z, z+h \in D_{1 / 2}$ :

$$
\left\|\left(w^{(I)}(\lambda, \sigma, z+h)-w^{(I)}(\lambda, \sigma, z)\right)-\partial_{z} w^{(I)}(\lambda, \sigma, z)\right\|^{\#}=o(1), \quad h \rightarrow 0 .
$$

First we need to show that $\partial_{z} w^{(I)}(\lambda, \sigma, z) \in \overline{\mathcal{W}}^{\#}$. To show this, consider the denominator occurring in the expressions for $w_{m, n}^{(I)}$,

$$
Q(r, a, z):=\frac{1}{r+2+a-z}
$$

where $a$ stands for $0,\left|k_{1}\right|,\left|\widetilde{k}_{1}\right|$ or $\left|k_{1}\right|+\left|\widetilde{k}_{1}\right|$. Now $\left|\partial_{r}^{s} \partial_{z} Q(r, a, z)\right| \leq|Q(r, a, z)|$ for $r \geq 0, z \in D_{1 / 2}$, and $s=0,1$. Thus $\partial_{z} w^{(I)}(\lambda, \sigma, z) \in \overline{\mathcal{W}}^{\#}$ follows from the corresponding estimate for $w^{(I)}$. To show (11.24) it suffices to consider the difference quotients of the denominators $Q(r, a, z)$,

$$
\frac{1}{h}(Q(r, a, z+h)-Q(r, a, z))-\partial_{z} Q(r, a, z)=h P(r, a, z, h),
$$

with

$$
P(r, a, z, h):=\frac{1}{(r+2+a+z)^{2}(r+2+a-z+h)} .
$$

If $h$ is sufficiently small than $\left|\partial_{r}^{s} P(r, a, z, h)\right| \leq 2|Q(r, a, z)|$ for $r \geq 0, z \in D_{1 / 2}$, and $s=0,1$. Thus (11.24), and hence the lemma, now follow again from the corresponding estimates for $w^{(I)}$.

(ii) of Theorem 11.3 follows from the convergence established in estimates (11.17)-(11.19), which is uniform in $(\sigma, z) \in[0, \infty) \times D_{1 / 2}$, and the following lemma:

Lemma 11.7. Let $|\lambda|<\mu_{0}$. Then the function $(\sigma, z) \mapsto V_{\underline{m}, \underline{p}, \underline{n}, \underline{q}}\left[w^{(I)}(\lambda, \sigma, z)\right]$ is a continuous function on $[0, \infty) \times D_{1 / 2}$ with respect to the $\|\cdot\|_{2}$-norm.

The proof uses essentially the same idea as the proof of Lemma 8.7. 
Proof. First observe that the kernel $V_{\underline{m}, \underline{p}, \underline{n}, \underline{q}}$ is a multi-linear expression of integral kernels; thus to show c-continuity we will use (8.12) and (7.3). Moreover, we will use the estimate

$$
\left[\int_{B_{1}^{m+n}} \frac{\mathrm{d} K^{(m, n)}}{\mid K^{\left.(m, n)\right|^{2}}} \sup _{r \geq 0}\left\|\bar{W}[w]_{p, q}^{m, n}\left(r, K^{(m, n)}\right)\right\|_{\mathrm{op}}^{2}\right]^{1 / 2} \leq\left\|w_{m+p, n+q}\right\|^{\sharp},
$$

with

$$
\begin{aligned}
\left\|w_{m, n}\right\|^{\sharp}:= & {\left[\int \frac{\mathrm{d} K^{(m, n)}}{\left|K^{(m, n)}\right|^{2}} \sup _{r \geq 0}\left|w_{m, n}\left(r, k^{(m)}, \widetilde{k}^{(n)}\right)\right|^{2}\right.} \\
& \left.\times\left(1+r+\Sigma\left[k^{(m)}\right]\right)^{m}\left(1+r+\Sigma\left[\widetilde{k}^{(n)}\right]\right)^{n}\right]^{1 / 2} .
\end{aligned}
$$

which follows from Lemma A.5. Above estimates together with the estimates in Lemma 11.5 and the limits (11.27) and (11.28), shown next, yield c-continuity. From the explicit expression of $w^{(I)}$ it follows from dominated convergence that for $m+n=2$

$$
\lim _{(z, \sigma) \rightarrow\left(z_{0}, \sigma_{0}\right)}\left\|w_{m, n}^{(I)}\left(\lambda, \sigma_{0}, z_{0}\right)-w_{m, n}^{(I)}(\lambda, \sigma, z)\right\|^{\sharp}=0,
$$

and it follows that

$$
\lim _{(z, \sigma) \rightarrow\left(z_{0}, \sigma_{0}\right)} \sup _{r \in[0, \infty)}\left|\frac{\bar{\chi}_{1}^{2}(r)}{t^{(I)}\left(\lambda, \sigma_{0}, z_{0}\right)(r)}-\frac{\bar{\chi}_{1}^{2}(r)}{t^{(I)}(\lambda, \sigma, z)(r)}\right|=0 .
$$

\section{Proving the Main Theorem}

In this section, we prove Theorem 2.4, the main result of this paper. The basic idea behind the proof of Theorem 2.4 is that the ground state and its energy are given as limits of uniformly convergent sequences having terms which are analytic in $\lambda$ and continuous in $\sigma$, respectively. A similar idea was used in [14] to show the analyticity in the coupling constant of the ground state and ground-state energy. The exposition in [14] is different due to the different representation of the spectral parameter.

Proof of Theorem 2.4. Choose $\rho, \xi, \epsilon_{0}$ such that Hypothesis (R) holds. By Theorem 11.3 we know that there exists a positive $\lambda_{0}$ which is less or equal than $\mu_{0}$ such that for all $\lambda \in D_{\lambda_{0}}$ and $\sigma \geq 0$ we have $w^{(0)}(\lambda, \sigma):=w^{(0)}(\lambda, \sigma, \cdot) \in$ $\mathcal{B}_{0}\left(\epsilon_{0} / 2, \epsilon_{0} / 2, \epsilon_{0} / 2\right)$, and that the analyticity, continuity and symmetry property as stated in (i)-(iii) of Theorem 11.3 hold. By Theorem 10.3 and a twofold application of the Feshbach property, see Theorem 4.2, it follows that $E_{\sigma}(\lambda)=e_{(0, \infty)}\left[w^{(0)}(\lambda, \sigma)\right]$ is an eigenvalue of $H_{\lambda, \sigma}$ with nonzero eigenvector $\psi_{\sigma}(\lambda)=Q_{\lambda, \sigma} Q_{\lambda, \sigma}^{(I)} \psi_{(0, \infty)}\left[w^{(0)}(\lambda, \sigma)\right]$ where

$$
\begin{aligned}
& Q_{\lambda, \sigma}:=Q_{\chi^{(I)}}\left(H_{\lambda, \sigma}-E_{\sigma}(\lambda), \tau+H_{f}-E_{\sigma}(\lambda)\right) \\
& Q_{\lambda, \sigma}^{(I)}:=Q_{\chi_{1}}\left(H_{\lambda, \sigma}^{(I)}\left(E_{\sigma}(\lambda)\right), T_{\lambda, \sigma}^{(I)}\left(E_{\sigma}(\lambda)\right)\right) .
\end{aligned}
$$


Note that the first two Feshbach transformations do not involve any transformation of the spectral parameter. By Theorem 10.4 it follows that $E_{\sigma}(\lambda)$ and $\psi_{(0, \infty)}\left[w^{(0)}(\lambda, \sigma)\right]$ are analytic in $\lambda \in D_{\lambda_{0}}$ for all $\sigma \geq 0$ and continuous in $\sigma \in[0, \infty)$ for all $\lambda \in D_{\lambda_{0}}$. It follows using Theorem 11.1 that $(\lambda, \sigma) \mapsto Q_{\lambda, \sigma}$ is uniformly bounded on $D_{\lambda_{0}} \times[0, \infty)$, analytic in $\lambda$, and continuous in $\sigma$ where the continuity follows from estimate (vi) in Lemma A.6. Similarly, it follows using Theorem 11.2 that $(\lambda, \sigma) \mapsto Q_{\lambda, \sigma}$ is uniformly bounded on $D_{\lambda_{0}} \times[0, \infty)$, analytic in $\lambda$, and continuous in $\sigma$ Now it follows that $\psi_{\sigma}(\lambda)$ is analytic for $\lambda \in D_{\lambda_{0}}$, continuous in $\sigma \geq 0$. Thus, we have shown (ii). Next we show that the expansion coefficients are bounded and continuous in $\sigma$ and use Cauchy's formula

$$
E_{\sigma}^{(n)}=\frac{1}{2 \pi i} \int_{|\lambda|=r} \frac{E_{\sigma}(\lambda)}{\lambda^{n+1}} \mathrm{~d} \lambda, \quad \psi_{\sigma}^{(n)}=\frac{1}{2 \pi i} \int_{|\lambda|=r} \frac{\psi_{\sigma}(\lambda)}{\lambda^{n+1}} \mathrm{~d} \lambda,
$$

for some positive $r$ which is less than $\lambda_{0}$. Using Cauchy's formula it follows that $\sup _{\sigma \geq 0}\left\|\psi_{\sigma}^{(n)}\right\|$ is bounded because of the uniform bound $\left\|\psi_{\sigma}(\lambda)\right\| \leq 4 \mathrm{e}^{4}$, see Theorem 10.3, and the boundedness of $Q_{\lambda, \sigma}$ and $Q_{\lambda, \sigma}^{(I)}$. Moreover, $\sigma \mapsto \psi_{\sigma}^{(n)}$ is continuous, which follows from Cauchy's formula and dominated convergence. Likewise, it follows that $\sup _{\sigma \geq 0}\left|E_{\sigma}^{(n)}\right|$ is bounded, since by definition $\left|E_{\sigma}(\lambda)\right| \leq 1 / 2$. Again using dominated convergence and Cauchy's formula we see that $\sigma \mapsto E_{\sigma}^{(n)}$ is continuous. By possibly choosing $\lambda_{0}$ smaller but still positive one can ensure that for all $\sigma \geq 0$ the projection

$$
P_{\sigma}(\lambda):=\frac{\left|\psi_{\sigma}(\lambda)\right\rangle\left\langle\psi_{\sigma}(\bar{\lambda})\right|}{\left\langle\psi_{\sigma}(\bar{\lambda}), \psi_{\sigma}(\lambda)\right\rangle}
$$

is well defined for $|\lambda|<\lambda_{0}$. To this end we need to show the uniformity in $\sigma$. By choosing a phase and a suitable normalization we can assume that $\psi_{\sigma}(\lambda)=\Omega_{\downarrow}+\sum_{n=1}^{\infty} \psi_{\sigma}^{(n)} \lambda^{n}$ with radius of convergence greater or equal to $\lambda_{0}$, where $\Omega_{\downarrow}$ is defined in (2.7). Since we have shown that $\psi_{\sigma}^{(n)}$ can be estimated uniformly in $\sigma \geq 0$, it follows that the denominator in (12.1) can be estimated from below by a positive constant uniformly in $\sigma \geq 0$. In view of (12.1), it follows that $\lambda \mapsto P_{\sigma}(\lambda)$ is an analytic function on $D_{\lambda_{0}}$ and that $P_{\sigma}(\lambda)^{*}=P_{\sigma}(\bar{\lambda})$. Thus we have shown (iii). The continuity of $P_{\sigma}^{(n)}$ in $\sigma$ follows from

$$
P_{\sigma}^{(n)}=\frac{1}{2 \pi i} \int_{|\lambda|=r} \frac{P_{\sigma}(\lambda)}{\lambda^{n+1}} \mathrm{~d} \lambda, \quad 0<r<\lambda_{0},
$$

dominated convergence, and in view of (12.1) the continuity of $\psi_{\sigma}(\lambda)$ in $\sigma$. To show (i) assume that $\lambda \in D_{\lambda_{0}}$ is real. Then $w^{(0)}(\lambda, \sigma)$ is a symmetric kernel, see Theorem 11.3. It now follows from Theorem 10.3 that $H_{\lambda, \sigma}^{(0)}(z)$ is bounded invertible if $z \in\left(-\frac{1}{2}, E_{\sigma}(\lambda)\right)$. Applying the Feshbach property twice it follows that $H_{\lambda}-z$ is bounded invertible for $z \in\left(-\frac{1}{2}, E_{\sigma}(\lambda)\right)$. For $z \leq-1 / 2$ the bounded invertibility of $H_{\sigma, \lambda}-z$ follows from the estimate $\left\|\lambda \sigma_{x} \phi\left(f_{\sigma}\right)\left(H_{f}+\tau+\frac{1}{2}\right)^{-1}\right\| \leq 6|\lambda| /\left(8 \mu_{0}\right)<1$, see Lemma A.6. Thus, 
$E_{\sigma}(\lambda)=\inf \sigma\left(H_{\sigma, \lambda}\right)$ for real $\lambda \in D_{\lambda_{0}} \cap \mathbb{R}$. The uniqueness follows from Theorem 2.3. Finally observe that $(-1)^{N} H_{\lambda, \sigma}(-1)^{N}=H_{-\lambda, \sigma}$ where $N$ is the closed linear operator on $\mathcal{F}$ with $N\left\lceil S_{n}\left(\mathfrak{h}^{\otimes n}\right)=n\right.$. This implies that the ground-state energy $E_{\sigma}(\lambda)$ cannot depend on odd powers of $\lambda$.

\section{Analytic Perturbation Theory}

In this section we discuss analytic perturbation theory. We put the discussion in Sect. 2 about analytic perturbation theory on a sound mathematical footing and justify Eq. (2.6), by proving Theorem 13.1 below. Moreover, we elaborate on Remark 2.6 at the end of this section.

Theorem 13.1. For $\sigma>0$, there is a $\lambda_{0}(\sigma)>0$ such that for all $\lambda \in D_{\lambda_{0}(\sigma)}$, the Hamiltonian $H_{\lambda, \sigma}$ has a non-degenerate eigenvalue $\widehat{E}_{\sigma}(\lambda)$ with eigen-projection $\widehat{P}_{\sigma}(\lambda)$ such that

(i) $\widehat{E}_{\sigma}(\lambda)=\inf \sigma\left(H_{\lambda, \sigma}\right)$ for $\lambda \in D_{\lambda_{0}(\sigma)}$ and $\widehat{E}_{\sigma}(0)=0$.

(ii) $\lambda \mapsto \widehat{E}_{\sigma}(\lambda)$ and $\lambda \mapsto \widehat{P}_{\sigma}(\lambda)$ are analytic functions on $D_{\lambda_{0}(\sigma)}$.

(iii) $\widehat{P}_{\sigma}^{*}(\lambda)=\widehat{P}_{\sigma}(\bar{\lambda})$ for all $\lambda \in D_{\lambda_{0}(\sigma)}$.

Moreover, on $D_{\lambda_{0}(\sigma)}$ we have a convergent power series expansion $\widehat{P}_{\sigma}(\lambda)=$ $\sum_{n=0}^{\infty} \widehat{P}_{\sigma}^{(n)} \lambda^{n}$, where $\widehat{P}_{\sigma}^{(n)}$ is given by $(2.6)$.

We will use the notation $P_{\downarrow}:=\left(\begin{array}{ll}0 & 0 \\ 0 & 1\end{array}\right)$.

Proof. Define the subspaces of $\mathfrak{h}$,

$$
\mathfrak{h}_{\sigma}^{(+)}:=L^{2}\left(\left\{k \in \mathbb{R}^{3}|| k \mid \geq \sigma\right\}\right), \quad \mathfrak{h}_{\sigma}^{(-)}:=L^{2}\left(\left\{k \in \mathbb{R}^{3}|| k \mid<\sigma\right\}\right)
$$

and the associated Fock-spaces $\mathcal{F}_{\sigma}^{( \pm)}:=\mathcal{F}\left(\mathfrak{h}_{\sigma}^{( \pm)}\right)$, where we denote the vacua by $\Omega_{\sigma}^{( \pm)}$. We consider the natural unitary isomorphism

$$
U: \mathcal{F}_{\sigma}^{(+)} \otimes \mathcal{F}_{\sigma}^{(-)} \rightarrow \mathcal{F}_{s}
$$

which is uniquely characterized by

$$
\begin{gathered}
U\left(S_{n}\left(h_{1} \otimes \cdots \otimes h_{n}\right) \otimes S_{m}\left(g_{1} \otimes \cdots \otimes g_{m}\right)\right) \\
\quad=S_{n+m}\left(h_{1} \otimes \cdots \otimes h_{n} \otimes g_{1} \otimes \cdots \otimes g_{m}\right),
\end{gathered}
$$

for any $h_{1}, \ldots, h_{n} \in \mathfrak{h}_{\sigma}^{(+)}$and $g_{1}, \ldots, g_{m} \in \mathfrak{h}_{\sigma}^{(-)}$. We denote the trivial extension of $U$ to $\mathbb{C}^{2} \otimes \mathcal{F}_{\sigma}^{(+)} \otimes \mathcal{F}_{\sigma}^{(-)}$by the same symbol. We write

$$
U^{*} H_{\sigma}(\lambda) U=\left(H_{0, \sigma}+\lambda T_{\sigma}^{(+)}\right) \otimes 1+1 \otimes 1 \otimes H_{f, \sigma}^{(-)},
$$

where we introduced the following operators acting on the corresponding spaces:

$$
\begin{aligned}
& H_{0, \sigma}:=\tau \otimes 1+1 \otimes H_{f, \sigma}^{(+)} \\
& T_{\sigma}^{(+)}:=\sigma_{x} \otimes \phi\left(\chi_{\sigma} f\right), \quad H_{f, \sigma}^{(+)}:=\mathrm{d} \Gamma\left(\chi_{\sigma} \omega\right), \quad H_{f, \sigma}^{(-)}:=\mathrm{d} \Gamma\left(\left(1-\chi_{\sigma}\right) \omega\right) .
\end{aligned}
$$


Now observe that $H_{f, \sigma}^{(-)}$has only one eigenvalue. That eigenvalue is zero, it is at the bottom of the spectrum, it is non-degenerate and its eigenvector is the vacuum of $\mathcal{F}_{\sigma}^{(-)}$. This implies that $H_{\sigma}(\lambda)$ and $H_{0, \sigma}+\lambda T_{\sigma}^{(+)}$have the same eigenvalues and the corresponding eigen-spaces are in bijective correspondence. Next observe that $H_{0, \sigma}$ has at the bottom of its spectrum an isolated non-degenerate eigenvalue. In fact, we have $\sigma\left(H_{0, \sigma}\right)=\{0\} \cup[\sigma, \infty)$. Moreover, $\lambda \mapsto H_{0, \sigma}+\lambda T_{\sigma}^{(+)}$is an analytic family, since the interaction term is infinitesimally bounded with respect to $H_{0, \sigma}$. Now by analytic perturbation theory, it follows that there exists an $\epsilon>0$ such that for $\lambda$ in a neighborhood of zero the following operator is well defined:

$$
P_{\sigma}^{(+)}(\lambda):=-\frac{1}{2 \pi i} \int_{|z|=\epsilon}\left(H_{0, \sigma}^{(+)}+\lambda T_{\sigma}^{(+)}-z\right)^{-1} \mathrm{~d} z .
$$

Moreover, the operator $P_{\sigma}^{(+)}(\lambda)$ projects onto a one-dimensional space which is the eigen-space of $H_{0, \sigma}^{(+)}+\lambda T_{\sigma}^{(+)}$with eigenvalue $E_{\sigma}(\lambda)$. Furthermore, $P_{\sigma}^{(+)}(\lambda)$ and $E_{\sigma}(\lambda)$ depend analytically on $\lambda$ and $E_{\sigma}(0)=0$. We conclude that $E_{\sigma}(\lambda)$ is a non-degenerate eigenvalue of $H_{\lambda, \sigma}$ with corresponding eigen-projection

$$
\widehat{P}_{\sigma}(\lambda)=U\left(P_{\sigma}^{(+)}(\lambda) \otimes P_{\Omega_{\sigma}^{(-)}}\right) U^{*},
$$

and properties (i)-(iii) of the theorem are satisfied, where $P_{\Omega_{\sigma}^{( \pm)}}$denotes the orthogonal projection in $\mathcal{F}_{\sigma}^{( \pm)}$onto $\Omega_{\sigma}^{( \pm)}$. Expanding in a Neumann series we find

$$
P_{\sigma}^{(+)}(\lambda)=-\sum_{n=0}^{\infty} \lambda^{n} \frac{1}{2 \pi i} \int_{|z|=\epsilon}\left(H_{0, \sigma}^{(+)}-z\right)^{-1}\left(T_{\sigma}^{(+)}\left(H_{0, \sigma}^{(+)}-z\right)^{-1}\right)^{n} \mathrm{~d} z .
$$

Inserting the Laurent expansion of the resolvent about 0 ,

$$
\left(H_{0, \sigma}^{(+)}-z\right)^{-1}=\sum_{\nu=0}^{\infty} S_{\sigma}^{(\nu,+)} z^{\nu-1}
$$

where

$$
S_{\sigma}^{(\nu,+)}= \begin{cases}-P_{\downarrow} \otimes P_{\Omega_{\sigma}^{(+)}}, & \nu=0 \\ \left(H_{0, \sigma}^{(+)}\right)^{-\nu}\left(1-P_{\downarrow} \otimes P_{\Omega_{\sigma}^{(+)}}\right), & \nu \geq 1,\end{cases}
$$

and calculating the contour integral we arrive at $P_{\sigma}^{(+)}(\lambda)=\sum_{n=0}^{\infty} P_{\sigma}^{(n,+)} \lambda^{n}$, with

$$
P_{\sigma}^{(n,+)}=-\sum_{\nu_{1}+\cdots+\nu_{n+1}=n, \nu_{i} \geq 0} S_{\sigma}^{\left(\nu_{1},+\right)} T_{\sigma}^{(+)} S_{\sigma}^{\left(\nu_{2},+\right)} \cdots T_{\sigma}^{(+)} S_{\sigma}^{\left(\nu_{n+1},+\right)} .
$$

Now using identity (13.2), we find (2.6) noting that $U\left(1 \otimes P_{\Omega_{\sigma}^{(-)}}\right) U^{*}=Q_{\sigma}$ and $U\left(S_{\sigma}^{(\nu,+)} \otimes P_{\Omega_{\sigma}^{(-)}}\right) U^{*}=S_{\sigma}^{(\nu)}$.

Next we will elaborate on the statement of Remark 2.6. We calculate the first four coefficients of $P(\lambda)$ using (2.6). We set $b(f)=a\left(f_{\sigma} / \sqrt{\omega}\right)$ and $b^{*}(f)=a^{*}\left(f_{\sigma} / \sqrt{\omega}\right)$. To keep the notation simple the integration symbol $\int$ 
stands in this section for $\prod_{i} \int_{\left|k_{i}\right| \geq \sigma}$ and we drop the $\sigma$-subscript of $\widehat{P}_{\sigma}^{(n)}, S_{\sigma}^{(\nu)}$, and $T_{\sigma}$. By divergent we mean an expression which diverges in the limit $\sigma \downarrow 0$. We will write $P_{\downarrow}$ for a shorthand notation of $P_{\downarrow} \otimes 1$ and define $P_{\Omega}:=1 \otimes|\Omega\rangle\langle\Omega|$. We find

$$
\widehat{P}^{(0)}=-S^{(0)}=P_{\downarrow} P_{\Omega}
$$

Furthermore, we have

$$
\begin{aligned}
\widehat{P}^{(1)} & =-S^{(1)} T S^{(0)}-S^{(0)} T S^{(1)} \\
& =\left(2+H_{f}\right)^{-1} b^{*}(f) P_{\downarrow} P_{\Omega}+P_{\Omega} P_{\downarrow} b(f)\left(2+H_{f}\right)^{-1} .
\end{aligned}
$$

To determine higher order expressions, we note that terms of the form $S^{(0)} T S^{(0)}$ vanish. We find

$$
P^{(2)}=-S^{(0)} T S^{(1)} T S^{(1)}-S^{(1)} T S^{(0)} T S^{(1)}-S^{(0)} T S^{(1)} T S^{(1)}-S^{(0)} T S^{(2)} T S^{(0)}
$$

with

$$
\begin{aligned}
& S^{(0)} T S^{(2)} T S^{(0)}=\int \frac{\mathrm{d}^{3} k|f(k)|^{2}}{(4 \pi)^{2}|k|(|k|+2)^{2}} P_{\downarrow} P_{\Omega} \\
& S^{(1)} T S^{(1)} T S^{(0)}=-H_{f}^{-1} b^{*}(f)\left(H_{f}+2\right)^{-1} b^{*}(f) P_{\downarrow} P_{\Omega} \\
& S^{(0)} T S^{(1)} T S^{(1)}=-P_{\downarrow} P_{\Omega} b(f)\left(H_{f}+2\right)^{-1} b(f) H_{f}^{-1} \\
& S^{(1)} T S^{(0)} T S^{(1)}=-P_{\uparrow}\left(H_{f}+2\right)^{-1} b^{*}(f) P_{\Omega} b(f)\left(H_{f}+2\right)^{-1} P_{\uparrow} .
\end{aligned}
$$

One checks that all above terms are not divergent. Similarly, one finds that $P^{(3)}$ does not contain any divergent terms. Next, we consider the following terms occurring in $\widehat{P}^{(4)}$ :

$$
A:=S^{(1)} T S^{(1)} T S^{(1)} T S^{(1)} T S^{(0)}, \quad B:=S^{(2)} T S^{(1)} T S^{(0)} T S^{(1)} T S^{(0)} .
$$

Inserting the definition of $T$ we find $A=A_{1}+A_{2}+A_{3}$ with

$$
\begin{aligned}
& A_{1}=-H_{f}^{-1} b(f)\left(H_{f}+2\right)^{-1} b^{*}(f) H_{f}^{-1} b^{*}(f)\left(H_{f}+2\right)^{-1} b^{*}(f) P_{\downarrow} P_{\Omega} \\
& A_{2}=-H_{f}^{-1} b^{*}(f)\left(H_{f}+2\right)^{-1} b(f) H_{f}^{-1} b^{*}(f)\left(H_{f}+2\right)^{-1} b^{*}(f) P_{\downarrow} P_{\Omega} \\
& A_{3}=-H_{f}^{-1} b^{*}(f)\left(H_{f}+2\right)^{-1} b^{*}(f) H_{f}^{-1} b^{*}(f)\left(H_{f}+2\right)^{-1} b^{*}(f) P_{\downarrow} P_{\Omega} .
\end{aligned}
$$

Earlier, we used that the other contributions to $A$ vanish, which can be seen by using the following identity which holds for $k \geq 1$ :

$$
S^{(k)}=H_{f}^{-k} P_{\Omega}^{\perp} P_{\downarrow}+\left(2+H_{f}\right)^{-k} P_{\Omega}^{\perp} P_{\uparrow}+2^{-k} P_{\uparrow} P_{\Omega},
$$

with $P_{\uparrow}:=1-P_{\downarrow}$ and $P_{\Omega}^{\perp}:=1-P_{\Omega}$. Using the pull-through formula and the canonical commutation relations, we find using $\mathrm{d} k^{(3)}=\mathrm{d}^{3} k_{1} \mathrm{~d}^{3} k_{2} \mathrm{~d}^{3} k_{3}$,

$$
\begin{aligned}
A_{1}= & -\int \frac{\mathrm{d} k^{(3)}\left|f\left(k_{1}\right)\right|^{2} f\left(k_{2}\right) f\left(k_{3}\right)}{(4 \pi)^{4}\left|k_{1}\right|\left|k_{2}\right|^{1 / 2}\left|k_{3}\right|^{1 / 2}}\left[\frac{1}{\left|k_{2}\right|+\left|k_{3}\right|}\right]\left[\frac{1}{\left|k_{1}\right|+\left|k_{2}\right|+\left|k_{3}\right|+2}\right] \\
& \times\left\{\frac{1}{\left|k_{2}\right|+\left|k_{3}\right|} \frac{1}{\left|k_{3}\right|+2}+\frac{1}{\left|k_{1}\right|+\left|k_{3}\right|} \frac{1}{\left|k_{3}\right|+2}+\frac{1}{\left|k_{3}\right|+\left|k_{1}\right|} \frac{1}{\left|k_{1}\right|+2}\right\} \\
& \times a^{*}\left(k_{2}\right) a^{*}\left(k_{3}\right) P_{\Omega} P_{\downarrow} .
\end{aligned}
$$


Note that only the first term in the brackets $\{\cdots\}$ yields a divergent expression. Similarly, one finds

$$
\begin{aligned}
A_{2}= & -\int \frac{\mathrm{d} k^{(3)}\left|f\left(k_{1}\right)\right|^{2} f\left(k_{2}\right) f\left(k_{3}\right)}{(4 \pi)^{4}\left|k_{1}\right|\left|k_{2}\right|^{1 / 2}\left|k_{3}\right|^{1 / 2}}\left[\frac{1}{\left|k_{2}\right|+\left|k_{3}\right|}\right]\left[\frac{1}{\left|k_{3}\right|+2}\right]\left[\frac{1}{\left|k_{1}\right|+\left|k_{3}\right|}\right] \\
& \times\left\{\frac{1}{\left|k_{3}\right|+2}+\frac{1}{\left|k_{1}\right|+2}\right\} a^{*}\left(k_{2}\right) a^{*}\left(k_{3}\right) P_{\Omega} P_{\downarrow} .
\end{aligned}
$$

One sees that $A_{2}$ is not divergent. Likewise, one checks that $A_{3}$ is not divergent. For $B$ we obtain

$$
\begin{aligned}
B= & \int \frac{\mathrm{d} k^{(3)}\left|f\left(k_{1}\right)\right|^{2} f\left(k_{2}\right) f\left(k_{3}\right)}{(4 \pi)^{4}\left|k_{1}\right|\left|k_{2}\right|^{1 / 2}\left|k_{3}\right|^{1 / 2}}\left[\frac{1}{\left|k_{2}\right|+\left|k_{3}\right|}\right]^{2}\left[\frac{1}{\left|k_{2}\right|+2}\right]\left[\frac{1}{\left|k_{1}\right|+2}\right] \\
& \times a^{*}\left(k_{2}\right) a^{*}\left(k_{3}\right) P_{\Omega} P_{\downarrow} .
\end{aligned}
$$

Now one sees that the divergence in $B$ and the divergence in $A$ cancel, which follows using the identity

$$
\frac{1}{\left|k_{1}\right|+2}-\frac{1}{\left|k_{1}\right|+\left|k_{2}\right|+\left|k_{3}\right|+2}=\frac{1}{\left|k_{1}\right|+2}\left(\left|k_{2}\right|+\left|k_{3}\right|\right) \frac{1}{\left|k_{1}\right|+\left|k_{2}\right|+\left|k_{3}\right|+2},
$$

and the symmetry of the corresponding expressions with respect to $k_{2}$ and $k_{3}$.

\section{Acknowledgements}

D.H. acknowledges the summer research grant of the College of William \& Mary. D.H. wants to thank ETH Zurich for hospitality. D.H. wants to thank G.M. Graf, J. Fröhlich, M. Griesemer, and A. Pizzo for interesting conversations.

\section{Appendix A. Elementary Estimates and Formulas}

To give a precise meaning to expressions which occur in (5.5) and (11.2), we introduce the following definition. For $\psi$ having finitely many particles we set

$$
\left[a\left(k_{1}\right) \cdots a\left(k_{l}\right) \psi\right]_{n}\left(k_{l+1}, \ldots, k_{l+n}\right):=\sqrt{\frac{(l+n) !}{n !}} \psi_{l+n}\left(k_{1}, \ldots, k_{l+n}\right),
$$

for $k_{1}, \ldots, k_{l+n} \in \mathbb{R}^{3}$. Using a theorem of Fubini it is elementary to see that for such $\psi$ the vector valued map $\left(k_{1}, \ldots, k_{l}\right) \mapsto a\left(k_{1}\right) \cdots a\left(k_{l}\right) \psi$ is an element of $L^{2}\left(\mathbb{R}^{3 l} ; \mathcal{F}\right)$. We note that definition (A.1) is consistent with $(2.1)$, since a straightforward computation shows that

$$
\begin{aligned}
& \int \overline{f_{1}}\left(k_{1}\right) \cdots \overline{f_{l}}\left(k_{l}\right)\left[a\left(k_{1}\right) \cdots a\left(k_{l}\right) \psi\right]_{n}\left(k_{l+1}, \ldots, k_{l+n}\right) \mathrm{d}^{3} k_{1} \cdots \mathrm{d}^{3} k_{l} \\
& \quad=\left[a\left(f_{1}\right) \cdots a\left(f_{l}\right) \psi\right]_{n}\left(k_{l+1}, \ldots, k_{l+n}\right) .
\end{aligned}
$$

We will make repeated use of the well known pull-through formula which is stated in the following lemma: 
Lemma A.1. Let $f: \mathbb{R}_{+} \rightarrow \mathbb{C}$ be a bounded measurable function. Then

$$
f\left(H_{f}\right) a^{*}(k)=a^{*}(k) f\left(H_{f}+\omega(k)\right), \quad a(k) f\left(H_{f}\right)=f\left(H_{f}+\omega(k)\right) a(k) .
$$

The pull-through formula can be shown as follows: Using definition (A.1), we have for any $\psi \in \mathcal{F}$ with finitely many particles

$$
\begin{aligned}
{[f} & \left.\left(H_{f}+\omega(k)\right) a(k) \psi\right]_{n}\left(k_{1}, \ldots, k_{n}\right) \\
& =f\left(\omega\left(k_{1}\right)+\cdots+\omega\left(k_{n}\right)+\omega(k)\right) \sqrt{n+1} \psi_{n+1}\left(k, k_{1}, \ldots, k_{n}\right) \\
& =\left[a(k) f\left(H_{f}\right) \psi\right]_{n}\left(k_{1}, \ldots, k_{n}\right) .
\end{aligned}
$$

This shows the second identity, since vectors with finitely many particles are dense in $\mathcal{F}$. The first identity follows by taking the adjoint of the second identity.

Remark A.2. Note that for $\psi \in \mathcal{H}_{\text {red }}$, the statements of Lemma A.3 and Corollary A.4 also hold in the case $X=B_{1}$.

Lemma A.3. Let $X=\mathbb{R}^{3}$ and $P_{\Omega}^{\perp}=1-|\Omega\rangle\langle\Omega|$. Then for $n \geq 1$,

$$
\begin{aligned}
& \int_{X^{n}} \mathrm{~d} k^{(n)}\left|k^{(n)}\right|\left\|\prod_{l=1}^{n}\left[H_{f}+\Sigma\left[k^{(l)}\right]\right]^{-1 / 2} a\left(k^{(n)}\right) \psi\right\|^{2} \\
& =\int_{X^{n}} \mathrm{~d} k^{(n)}\left|k^{(n)}\right|\left\|\prod_{l=1}^{n}\left[a\left(k_{l}\right) H_{f}^{-1 / 2}\right] P_{\Omega}^{\perp} \psi\right\|^{2} \\
& =\left\|P_{\Omega}^{\perp} \psi\right\|^{2} .
\end{aligned}
$$

Proof. The first identity follows from the pull-through formula. The second identity follows from iteration of the following equality where $\phi=P_{\Omega}^{\perp} \psi$ :

$$
\int_{X} \mathrm{~d}^{3} k|k|\left\|a(k) H_{f}^{-1 / 2} \phi\right\|^{2}=\left\langle H_{f}^{-1 / 2} \phi, H_{f} H_{f}^{-1 / 2} \phi\right\rangle=\|\phi\|^{2} .
$$

Corollary A.4. Let $X=\mathbb{R}^{3}$. Then,

$$
\int_{X^{n}} \mathrm{~d} k^{(n)}\left|k^{(n)}\right|\left\|a\left(k^{(n)}\right) \psi\right\|^{2} \leq\left\|H_{f}^{n / 2} \psi\right\|^{2} .
$$

Proof.

$$
\begin{array}{rl}
\int_{X^{n}} & \mathrm{~d} k^{(n)}\left|k^{(n)}\right|\left\|a\left(k^{(n)}\right) \psi\right\|^{2} \\
& =\int_{X^{n}} \mathrm{~d} k^{(n)}\left|k^{(n)}\right|\left\|\left(H_{f}+\Sigma\left[k^{(n)}\right]\right)^{-n / 2} a\left(k^{(n)}\right) H_{f}^{n / 2} \psi\right\|^{2} \\
& \leq \int_{X^{n}} \mathrm{~d} k^{(n)}\left|k^{(n)}\right|\left\|\prod_{l=1}^{n}\left[H_{f}+\Sigma\left[k^{(l)}\right]\right]^{-1 / 2} a\left(k^{(n)}\right) H_{f}^{n / 2} \psi\right\|^{2} \\
= & \left\|H_{f}^{n / 2} \psi\right\|^{2},
\end{array}
$$


where in the first equality we used the pull-through formula. The second line follows from an elementary operator inequality using $\Sigma\left[k^{(l)}\right] \leq \Sigma\left[k^{(n)}\right]$, if $l \leq n$. The last equality follows from Lemma A.3.

Lemma A.5. For $\bar{H}_{m, n}(\cdot)$ as defined in (11.2) we have

$$
\begin{aligned}
& \left\|\bar{H}_{m, n}\left(w_{m, n}\right)\right\|^{2} \\
& \leq \int \frac{\mathrm{d} K^{(m, n)}}{\mid K^{\left.(m, n)\right|^{2}}} \sup _{r \geq 0}\left|w_{m, n}\left(r, K^{(m, n)}\right)\right|^{2} \prod_{l=1}^{m}\left[r+\Sigma\left[k^{(l)}\right]\right] \prod_{\widetilde{l}=1}^{n}\left[r+\Sigma\left[\widetilde{k}^{(\widetilde{l})}\right]\right] \\
& \quad \leq \int \frac{\mathrm{d} K^{(m, n)}}{\left|K^{(m, n)}\right|^{2}} \sup _{r \geq 0}\left|w_{m, n}\left(r, K^{(m, n)}\right)\right|^{2}\left[r+\Sigma\left[k^{(m)}\right]\right]^{m}\left[r+\Sigma\left[\widetilde{k}^{(n)}\right]\right]^{n}
\end{aligned}
$$

Proof. The first inequality is obtained by estimating

$$
\left|\left\langle\phi, \bar{H}_{m, n}\left(w_{m, n}\right) \psi\right\rangle\right| \text {. }
$$

To this end one inserts identities of the form $1=\left[H_{f}+\Sigma\left[k^{(l)}\right]\right]^{1 / 2}$ $\left[H_{f}+\Sigma\left[k^{(l)}\right]\right]^{-1 / 2}$, uses the Cauchy-Schwarz inequality, and Lemma A.3. The second inequality follows from the first since $\Sigma\left[k^{(p)}\right] \leq \Sigma\left[k^{(q)}\right]$, if $p \leq q$.

Now we collect some elementary estimates.

Lemma A.6. We have the estimates

(i) $\|a(f) \varphi\| \leq\|f / \sqrt{\omega}\|\left\|H_{f}^{1 / 2} \varphi\right\|$

(ii) $\left\|H_{f}^{-1 / 2} P_{\Omega}^{\perp} a^{*}(f)\right\|=\left\|a(f) H_{f}^{-1 / 2} P_{\Omega}^{\perp}\right\| \leq\|f / \sqrt{\omega}\|$

(iii) $\left\|a^{*}(f) \varphi\right\|^{2} \leq\|f\|^{2}\|\varphi\|^{2}+\|f / \sqrt{\omega}\|^{2}\left\|H_{f}^{1 / 2} \varphi\right\|^{2}$

(iv) $\left\|a(f)\left(H_{f}+1\right)^{-1 / 2}\right\|=\left\|\left(H_{f}+1\right)^{-1 / 2} a^{*}(f)\right\| \leq\|f / \sqrt{\omega}\|$

(v) $\quad\left\|\left(H_{f}+1\right)^{-1 / 2} a(f)\right\|=\left\|a^{*}(f)\left(H_{f}+1\right)^{-1 / 2}\right\| \leq\left(\|f / \sqrt{\omega}\|^{2}+\|f\|^{2}\right)^{1 / 2}$

(vi) $\quad\left\|\left(H_{f}+1\right)^{-1 / 2} \phi(f)\right\|=\left\|\phi(f)\left(H_{f}+1\right)^{-1 / 2}\right\| \leq\left\|\frac{f}{4 \pi \sqrt{\omega}}\right\|+\left[\left\|\frac{f}{4 \pi \omega}\right\|^{2}\right.$ $\left.+\left\|\frac{f}{4 \pi \sqrt{\omega}}\right\|^{2}\right]^{1 / 2}$

Proof. (i) follows from Corollary A.4 with $n=1$ and the Cauchy-Schwarz inequality, (ii) follows from (i), (iii) follows from (i) and the canonical commutation relations of the creation and annihilation operators, (iv) follows from (ii), (v) follows from (iii), and (vi) follows from (iv) and (v).

\section{Appendix B. Wick's Theorem}

Let $\left(\sigma_{1}, \sigma_{2}, \ldots, \sigma_{n}\right\} \in\{+,-\}^{n}$. For any subset $Z \subset N_{n}:=\{1,2, \ldots, n\}, n \in \mathbb{N}$, we set $Z_{ \pm}:=\left\{j \in Z \mid \sigma_{j}= \pm\right\}$. We use the notation $a^{+}(k)=a^{*}(k)$ and $a^{-}(k)=a(k)$. We define the Wick-ordered product $: \cdot:$ by

$$
: \prod_{j \in Z} a^{\sigma_{j}}\left(k_{j}\right)::=\prod_{j \in Z_{+}} a^{\sigma_{j}}\left(k_{j}\right) \prod_{j \in Z_{-}} a^{\sigma_{j}}\left(k_{j}\right)
$$


We introduce a notation for the vacuum expectation of an operator $A$ on Fock space by setting

$$
\langle A\rangle:=\langle\Omega \mid A \Omega\rangle \text {. }
$$

Lemma B.1. (Wick's Theorem) For any $\left(\sigma_{1}, \sigma_{2}, \ldots, \sigma_{n}\right) \in\{+,-\}^{n}$

$$
\prod_{j \in N_{n}} a^{\sigma_{j}}\left(k_{j}\right)=\sum_{Z \subset N_{n}}\left\langle\prod_{j \in N_{n} \backslash Z} a^{\sigma_{j}}\left(k_{j}\right)\right\rangle: \prod_{j \in Z} a^{\sigma_{j}}\left(k_{j}\right):
$$

See, for example [21] (Chapter 13, Eq. (106) and references therein). The next lemma is from [5]. It follows from an application of Wick's theorem and the pull-through formula.

Lemma B.2. Let $f_{j}$ be measurable functions on $\mathbb{R}_{+}$. Then,

$$
\begin{aligned}
& \prod_{j=1}^{n}\left\{a^{\sigma_{j}}\left(k_{j}\right) f_{j}\left(H_{f}\right)\right\}=\sum_{Z \subset N_{n}} \\
& \quad \times\left.\prod_{j \in Z_{+}} a^{+}\left(k_{j}\right)\left\langle\prod_{j=1}^{n}\left\{\left[a^{\sigma_{j}}\left(k_{j}\right)\right]^{\chi_{Z^{c}}(j)} f_{j}\left(H_{f}+r+\omega_{j}\right)\right\}\right\rangle\right|_{r=H_{f}} \prod_{j \in Z_{-}} a^{-}\left(k_{j}\right),
\end{aligned}
$$

where we set

$$
\omega_{j}=\sum_{\substack{i=1 \\ i \in Z_{-}}}^{j}\left|k_{i}\right|+\sum_{\substack{i=j+1 \\ i \in Z_{+}}}^{n}\left|k_{i}\right|
$$

Proof of Theorem 7.2. For $m+n \geq 1$ we set

$$
\widehat{W}_{m, n}=\int a^{*}\left(z^{(m)}\right) w\left(H_{f}, Z^{(m, n)}\right) a\left(\widetilde{z}^{(n)}\right) \frac{\mathrm{d} Z^{(m, n)}}{\mid Z^{(m, n) \mid 1 / 2}} .
$$

Using Lemma B.2 and the observation that there are

$$
\left(\begin{array}{c}
m_{l}+p_{l} \\
p_{l}
\end{array}\right)
$$

ways to choose $p_{l}$ elements out of a set containing $m_{l}+p_{l}$ elements, we find

$$
\begin{aligned}
& F_{0}\left(H_{f}\right) \widehat{W}_{M_{1}, N_{1}} F_{1}\left(H_{f}\right) \widehat{W}_{M_{2}, N_{2}} \cdots \widehat{W}_{M_{L}, N_{L}} F_{L}\left(H_{f}\right) \\
& =\sum_{\begin{array}{c}
p_{1}, \ldots, p_{L}, m_{1}, \ldots, m_{L}, \\
q_{1}, \ldots, q_{L}, n_{1}, \ldots, n_{L}: \\
p_{i}+m_{i}=M_{i}, q_{i}+n_{i}=N_{i}, \\
p_{l}+q_{l}+m_{l}+n_{l} \geq 1
\end{array}}\left(\begin{array}{c}
m_{l}+p_{l} \\
p_{l}
\end{array}\right)\left(\begin{array}{c}
n_{l}+q_{l} \\
q_{l}
\end{array}\right) \int a^{*}\left(k_{1}^{\left(m_{1}\right)}\right) \cdots a^{*}\left(k_{L}^{\left(m_{L}\right)}\right) \\
& \quad\left\langle\Omega, F_{0}\left(H_{f}+r+\widetilde{r}_{0}\right) \widehat{W}_{1}\left(r+r_{1}, K_{1}^{\left(m_{1}, n_{1}\right)}\right) F_{1}\left(H_{f}+r+\widetilde{r}_{1}\right)\right. \\
& \quad \widehat{W}_{2}\left(r+r_{2}, K_{2}^{\left(m_{2}, n_{2}\right)}\right) \cdots F_{L-1}\left(H_{f}+r+\widetilde{r}_{L-1}\right) \widehat{W}_{L}\left(r+r_{L}, K_{L}^{\left(m_{L}, n_{L}\right)}\right) \\
& \left.\quad F_{L}\left(H_{f}+r+\widetilde{r}_{L}\right) \Omega\right\rangle\left.\right|_{r=H_{f}} a\left(\widetilde{k}_{1}^{\left(n_{1}\right)}\right) \cdots a\left(\widetilde{k}_{L}^{\left(n_{L}\right)}\right) \prod_{j=1}^{L} \frac{\mathrm{d} K_{j}^{\left(m_{j}, n_{j}\right)}}{\left|K_{j}^{\left(m_{j}, n_{j}\right)}\right|^{1 / 2}}
\end{aligned}
$$


where we have set $\widehat{W}_{i}=\widehat{W}_{p_{i}, q_{i}}^{m_{i}, n_{i}}$ with

$$
\begin{aligned}
& \widehat{W}_{p_{l}, q_{l}}^{m_{l}, n_{l}}[w]\left(r, K_{l}^{\left(m_{l}, n_{l}\right)}\right)=P_{\text {red }} \int_{B_{1}^{p_{l}+q_{l}}} \frac{\mathrm{d} X^{\left(p_{l}, q_{l}\right)}}{\mid X^{\left.\left(p_{l}, q_{l}\right)\right|^{1 / 2}}} \\
& \quad \times a^{*}\left(x^{\left(p_{l}\right)}\right) w_{p_{l}+m_{l}, q_{l}+n_{l}}\left(H_{f}+r, x^{\left(p_{l}\right)}, k_{l}^{\left(m_{l}\right)}, \widetilde{x}^{\left(q_{l}\right)},{\widetilde{k_{l}}}^{\left(n_{l}\right)}\right) a\left(\widetilde{x}^{\left(q_{l}\right)}\right) P_{\text {red }} .
\end{aligned}
$$

With this notation we have used the permutation symmetry of $w_{m, n}$ and

$$
\mathrm{d} Z_{l}^{\left(M_{l}, N_{l}\right)}=\mathrm{d} X^{\left(p_{l}, q_{l}\right)} \mathrm{d} K_{l}^{\left(m_{l}, n_{l}\right)} .
$$

Summing over all $\left(M_{i}, N_{i}\right)$, it is easy to read off the kernels of the resulting operator, $H\left(\widetilde{w}^{(\mathrm{sym})}\right)$.

\section{References}

[1] Arai, A., Hirokawa, M.: On the existence and uniqueness of ground states of a generalized spin-boson model. J. Funct. Anal. 151, 455-503 (1997)

[2] Bach, V., Chen, T., Fröhlich, J., Sigal, I.M.: Smooth Feshbach map and operator-theoretic renormalization group methods. J. Funct. Anal. 203, 44-92 (2003)

[3] Bach, V., Fröhlich, J., Pizzo, A.: Infrared-finite algorithms in QED: the groundstate of an atom interacting with the quantized radiation field. Commun. Math. Phys. 264, 145-165 (2006)

[4] Bach, V., Fröhlich, J., Pizzo, A.: Infrared-finite algorithms in QED. II. The expansion of the groundstate of an atom interacting with the quantized radiation field. Adv. Math. 220, 1023-1074 (2009)

[5] Bach, V., Fröhlich, J., Sigal, I.M.: Renormalization group analysis of spectral problems in quantum field theory. Adv. Math. 137, 205-298 (1998)

[6] Bach, V., Fröhlich, J., Sigal, I.M.: Spectral analysis for systems of atoms and molecules coupled to the quantized radiation field. Commun. Math. Phys. 207, 249290 (1999)

[7] Barbaroux, J-M., Chen, T., Vugalter, S., Vougalter, V.: Quantitative estimates on the Hydrogen ground state energy in non-relativistic QED. mp_arc 09-48. Ann. Henri Poincar. 11(8), 1487-1544 (2010)

[8] Catto, I., Hainzl, C.: Self-energy of one electron in non-relativistic QED. J. Funct. Anal. 207, 68-110 (2004)

[9] Dixmier, J.: Von Neumann Algebras. Translated from the second French edition by F. Jellett. North-Holland Mathematical Library, vol. 27. North-Holland Publishing Co., Amsterdam (1981)

[10] Griesemer, M., Lieb, E., Loss, M.: Ground states in non-relativistic quantum electrodynamics. Invent. Math. 145, 557-595 (2001)

[11] Fröhlich, J.: On the infrared problem in a model of scalar electrons and massless, scalar bosons. Ann. Inst. H. Poincar Sect. A (N.S.) 19, 1-103 (1973)

[12] Gérard, C.: On the existence of ground states for massless Pauli-Fierz Hamiltonians. Ann. Henri Poincaré 1, 443-459 (2000)

[13] Griesemer, M., Hasler, D.: On the smooth Feshbach-Schur Map. J. Funct. Anal. 254, 2329-2335 (2008) 
[14] Griesemer, M., Hasler, D.: Analytic perturbation theory and renormalization analysis of matter coupled to quantized radiation. Ann. Henri Poincaré 10, 577$621(2009)$

[15] Hainzl, C., Hirokawa, M., Spohn, H.: Binding energy for hydrogen-like atoms in the Nelson model without cutoffs. J. Funct. Anal. 220, 424-459 (2005)

[16] Hasler, D., Herbst, I.: Absence of ground states for a class of translation invariant models of non-relativistic QED. Commun. Math. Phys. 279, 769-787 (2008)

[17] Hübner, M., Spohn, H.: Spectral properties of the spin-boson Hamiltonian. Ann. Inst. H. Poincaré Phys. Théor. 62, 289-323 (1995)

[18] Kato, T.: Perturbation Theory for Linear Operators. pp. 75-80. SpringerVerlag, New York (1966)

[19] Lörinczi, J., Minlos, R.A., Spohn, H.: The infrared behaviour in Nelson's model of a quantum particle coupled to a massless scalar field. Ann. Henri Poincaré 3(2), 269-295 (2002)

[20] Reed, M., Simon, B.: Methods of Modern Mathematical Physics. IV. Analysis of Operators. Academic Press, New York (1978)

[21] Schweber S.: An Introduction to Relativistic Quantum Field Theory. Foreword by Hans A. Bethe. Row, Peterson and Company, Evanston (1961)

[22] Simon, B.: The $P(\phi)_{2}$ Euclidean (quantum) Field Theory. Princeton Series in Physics. Princeton University Press, Princeton (1974)

[23] Spohn, H., Dümcke, R.: Quantum tunneling with dissipation and the Ising model over R. J. Stat. Phys. 41, 389-423 (1985)

[24] Spohn, H.: Ground state(s) of the spin-boson Hamiltonian. Commun. Math. Phys. 123, 277-304 (1989)

[25] Spohn, H.: Ground state of a quantum particle coupled to a scalar Bose field. Lett. Math. Phys. 44, 9-16 (1998)

David Hasler

Department of Mathematics

College of William and Mary

Williamsburg

VA 23187-8795

USA

e-mail: dghasler@wm.edu

Ira Herbst

Department of Mathematics

University of Virginia

Charlottesville

VA 22904-4137

USA

e-mail: iwh@virginia.edu

Communicated by Claude Alain Pillet.

Received: April 7, 2010.

Accepted: September 14, 2010. 